Novos exemplos de NS-pares e de fibrações de Milnor reais não-triviais 



\title{
Novos exemplos de NS-pares e de fibrações de Milnor reais não-triviais
}

\author{
Maria Amelia de Pinho Barbosa Hohlenwerger
}

Orientador: Prof. Dr. Raimundo Nonato Araújo dos Santos

Tese apresentada ao Instituto de Ciências Matemáticas e de Computação - ICMC-USP, como parte dos requisitos para obtenção do título de Doutor em Ciências - Matemática. VERSÃO REVISADA 
Ficha catalográfica elaborada pela Biblioteca Prof. Achille Bassi e Seção Técnica de Informática, ICMC/USP, com os dados fornecidos pelo(a) autor(a)

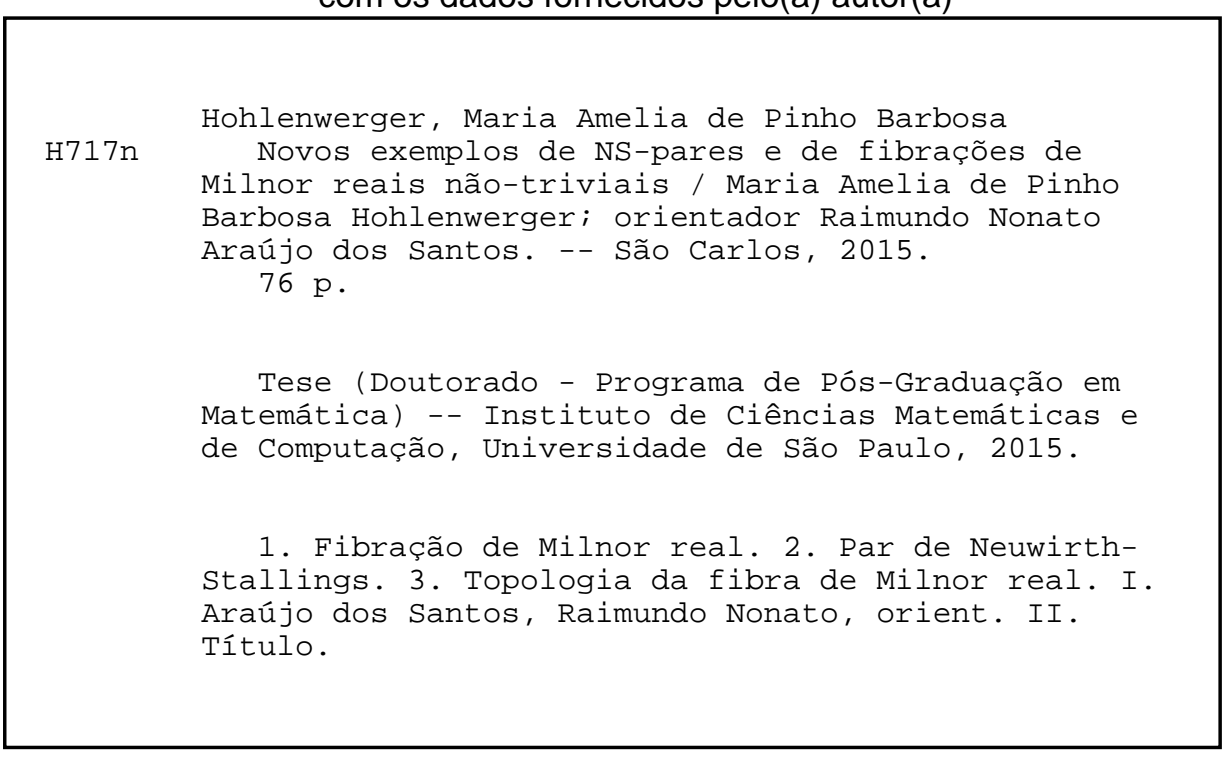


"Há um tempo em que é preciso abandonar as roupas usadas, que já têm a forma do nosso corpo, e esquecer os nossos caminhos, que nos levam sempre aos mesmos lugares. É

o tempo da travessia: e, se não ousarmos fazê-la, teremos ficado, para sempre, à margem de nós mesmos." Fernando Teixeira de Andrade 
Ao meu esposo Amasis, e aos meus

filhos, Matheus, Beatriz e Pedro, dedico. 


\section{Agradecimentos}

Durante esses quatro anos, ouvi muitas pessoas dizerem que eu era corajosa por decidir fazer Doutorado com três filhos para criar... Acho que não se trata de coragem e sim de felicidade, sentimento que nos invade quando alcançamos um importante objetivo, quando realizamos um sonho, quando vencemos. Que estejamos sempre motivados a encontrar este lindo sentimento. A seguir, tentarei expressar meu agradecimento àqueles que estão relacionados com a realização deste trabalho e que, consequentemente, estão relacionados com a minha felicidade.

A Deus, por me abençoar, me guiar, iluminar meus caminhos e sempre me dar forças.

À minha amada família, por aceitar fazer uma grande mudança para estar perto de mim, me dando alegrias. Em especial ao meu esposo Amasis, pelo companheirismo, paciência e apoio, os quais foram essenciais para o desenvolvimento e finalização deste projeto. Sem a dedicação da minha família, tudo teria sido muito difícil.

Ao meu orientador Prof. Raimundo N. Santos, pelo exemplo de como ser pesquisador, pela compreensão, amizade e pelos incentivos.

Aos Professores Denise de Mattos, Edivaldo Santos, Osamu Saeki, Taciana Souza e Oziride Neto, pelas frutíferas discussões e importantes contribuições e comentários e novamente ao Professor Saeki pela oportunidade de conhecer o Japão, aprender um pouco da sua cultura e discutir Matemática na Universidade de Kyushu.

Aos meus pais, Almir e Nilda, e à minha sogra, Jesse, pelo amor, pelos ensinamentos, incentivos e torcida por uma conclusão deste trabalho com êxito, para que eu voltasse logo para a Bahia.

Aos meus irmãos e suas respectivas famílias, por todas as palavras de carinho e incentivo.

Ao CETEC/UFRB, especialmente aos meus colegas que aceitaram assumir mais turmas, pela oportunidade de ser liberada das minhas atividades para realizar este projeto.

Aos meus colegas da pós-graduação, pelos estudos em grupo, discussões de assuntos variados, pelas companhias nos eventos científicos, confraternizações, viagens, refeições e 
etc, pelos momentos de descontração. Enfim, por fazerem parte dessa minha história.

Aos professores e funcionários do ICMC/USP, pela presteza e apoio.

A todos aqueles que contribuíram, de alguma forma, na realização deste projeto.

À CAPES (Prodoutoral) e à FAPESP (processo no 2012/12972-4), pelo apoio financeiro. 


\section{Resumo}

Neste trabalho, nos concentramos no estudo da topologia da fibração de Milnor associada a um germe de aplicação polinomial $f:\left(\mathbb{R}^{n}, 0\right) \rightarrow\left(\mathbb{R}^{p}, 0\right)$ com uma singularidade isolada na origem. O primeiro resultado é uma extensão da caracterização de germes de aplicações triviais nos pares de dimensões $(n, p)$ quando $n-p=3$. Uma caracterização inicial foi apresentada por Church e Lamotke em 1975. O segundo resultado é a caracterização de NS-pares $\left(S^{5}, K^{2}\right)$, usando a topologia de espaços de configuração. Como uma consequência desta caracterização, mostramos a existência de germe de aplicação polinomial real nos pares de dimensões $(6,3)$ com uma singularidade isolada na origem tal que sua fibra de Milnor não é difeomorfa a um disco. A existência desses exemplos coloca um fim ao problema da não-trivialidade proposto por Milnor em 1968 e além disso, nos permite apresentar um novo resultado sobre a topologia da fibra de Milnor real nos pares de dimensões $(2 n, n)$ e $(2 n+1, n), n \geqslant 3$. Tal resultado garante a existência de germes de aplicações polinomiais $\left(\mathbb{R}^{n}, 0\right) \rightarrow\left(\mathbb{R}^{p}, 0\right), n \geqslant p \geqslant 2$, com uma singularidade isolada na origem tais que suas fibras de Milnor têm o tipo de homotopia de um buquê de um número positivo de esferas.

Palavras-chave: topologia das fibras reais, fibra de Milnor real, link fibrado, germe de aplicação polinomial real, par de Neuwirth-Stallings, espaço de configuração. 


\section{Abstract}

In this work, we focus on the study of the topology of the Milnor fibration associated with a polynomial map germ $f:\left(\mathbb{R}^{n}, 0\right) \rightarrow\left(\mathbb{R}^{p}, 0\right)$ with an isolated singularity at the origin. The first result is an extension of the characterization of trivial map germs in the pairs of dimensions $(n, p)$ when $n-p=3$. An initial characterization was presented by Church and Lamotke in 1975. The second result is a characterization of NS-pairs $\left(S^{5}, K^{2}\right)$, using the topology of configuration spaces. As a consequence of this characterization, we show the existence of real polynomial map germs in the pairs of dimensions $(6,3)$ with an isolated singularity at the origin such that its Milnor fibers are not diffeomorphic to a disc. The existence of such examples ends a non-triviality problem posed by Milnor in 1968 and furthermore, it allows us to show a new result about the topology of the real Milnor fibers in the pairs of dimensions $(2 n, n)$ and $(2 n+1, n), n \geqslant 3$. This result ensure the existence of polynomial map germs $\left(\mathbb{R}^{n}, 0\right) \rightarrow\left(\mathbb{R}^{p}, 0\right), n \geqslant p \geqslant 2$, with an isolated singularity at the origin such that its Milnor fibers has the homotopy type of a bouquet of a positive number of spheres.

Key words and phrases: topology of the real fibers, real Milnor fiber, fibered link, real polynomial map germ, Neuwirth-Stallings pair, configuration space. 



\section{Sumário}

Introdução 1

1 Preliminares 5

1.1 Ferramentas da Topologia Algébrica . . . . . . . . . . . . . 5 5

1.1.1 Colagem de Espaços . . . . . . . . . . . . . . . . . . . . . . 8

1.2 Ferramentas da Topologia Diferencial . . . . . . . . . . . . . . . . . . . . 9

1.2.1 Fibrados e Fibrações . . . . . . . . . . . . . . . . . . . . . . . 9

1.2.2 O grau de Brouwer . . . . . . . . . . . . . . . . . . 14

1.2.3 Transversalidade . . . . . . . . . . . . . . . . . . . . . . 15

1.2.4 h-cobordismo e aplicações . . . . . . . . . . . . . . 15

1.3 Fórmulas do tipo Poincaré-Hopf . . . . . . . . . . . . . . . . . . . 17

1.4 Grupos de Lie . . . . . . . . . . . . . . . . . . . . . . . . . . . 18

2 Fibrados suaves 23

2.1 A fibração de Milnor: abordagem moderna . . . . . . . . . . . . . . . 23

2.2 A topologia da fibra e do link . . . . . . . . . . . . . . . 31

2.3 Sobre os questionamentos de Milnor . . . . . . . . . . . . . . . . . . 35

2.4 Nós, links e NS-pares . . . . . . . . . . . . . . . . . . . 40

2.5 Linking number e espaço de configuração . . . . . . . . . . . . . . . . . . . 44

3 Caracterização de aplicações triviais com fibras de Milnor 3-dimensionais 45

3.1 Prova do Teorema 3.0.3 . . . . . . . . . . . . . . . . . 47

$3.1 .1 \quad$ o caso $(6,3) \ldots \ldots \ldots \ldots \ldots \ldots$

$3.1 .2 \quad$ o caso $(8,5) \ldots \ldots \ldots \ldots \ldots \ldots$ 
3.1 .3 o caso $(5,2) \ldots \ldots \ldots \ldots \ldots$

4 Novos exemplos de NS-pares $\quad 51$

4.1 Classificação de fibrados . . . . . . . . . . . . . . . . . . . 51

4.2 Caracterização de NS-pares . . . . . . . . . . . . . . . 54

4.3 Uma generalização para dimensões altas . . . . . . . . . . . . . . . 61

5 Novos resultados sobre a topologia da fibra de Milnor real 65

5.1 Spinning para nó fibrado . . . . . . . . . . . . . . . 65

5.2 Buquê de esferas - caso complexo . . . . . . . . . . . . . . 66

5.3 Teorema do Buquê - caso real . . . . . . . . . . . . . . 67

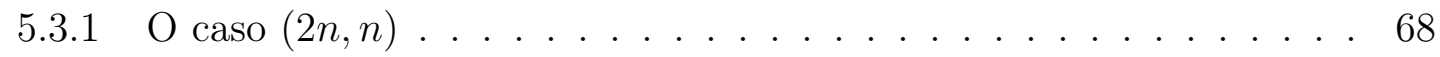

5.3 .2 O caso $(2 n+1, n) \ldots \ldots \ldots \ldots \ldots$

5.4 Aplicação $k$-stairs . . . . . . . . . . . . . . . . . . . 72

$\begin{array}{ll}\text { Referências Bibliográficas } & 76\end{array}$ 


\section{Introdução}

A existência da fibração de Milnor numa vizinhança de um ponto singular é uma ferramenta bastante utilizada no estudo da estrutura topológica da singularidade. Essa ferramenta passou a ser conhecida em 1968, com o lançamento do livro "Singular Points of Complex Hypersurfaces", de autoria de John Milnor. Neste livro, o autor demonstra dois teoremas de fibração: um para função analítica complexa e outro para aplicação analítica real.

No caso complexo, ele mostrou que dado um representante de um germe de função holomorfa $\psi: U \subset \mathbb{C}^{n+1} \rightarrow \mathbb{C}$ com $\psi(0)=0$, existe um número real suficientemente pequeno $\varepsilon_{0}>0$ tal que para todo $0<\varepsilon \leqslant \varepsilon_{0}$,

$$
\frac{\psi}{\|\psi\|}: S_{\varepsilon}^{2 n+1} \backslash K \rightarrow S^{1}
$$

é uma projeção suave de um fibrado localmente trivial, onde $S_{\varepsilon}^{2 n+1}$ é a esfera $(2 n+1)$ dimensional de raio $\varepsilon$, centrada na origem e

$$
K=\psi^{-1}(0) \cap S_{\varepsilon}^{2 n+1}
$$

é chamado o link da singularidade na origem.

Denotando por $F_{\theta}=\left(\frac{\psi}{\|\psi\|}\right)^{-1}\left(e^{i \theta}\right)$ a fibra de (0.0.1), onde $e^{i \theta} \in S^{1}$, e por $\bar{F}_{\theta}=F_{\theta} \cup K$ o seu fecho topológico, Milnor deu detalhes sobre a topologia da fibra e do link e provou que se $0 \in \mathbb{C}^{n+1}$ é um ponto singular isolado de $\psi$, então a fibra $F_{\theta}$ é paralelizável ${ }^{1}$, $(n-1)$-conexa e tem o tipo de homotopia de um buquê de esferas $S^{n} \vee \ldots \vee S^{n}$, sendo o número de esferas do buquê chamado, posteriormente, o número de Milnor.

No caso real, considere uma aplicação polinomial $f: \mathbb{R}^{n} \rightarrow \mathbb{R}^{p}$ tal que $f(0)=0$, com $n \geqslant p \geqslant 2$ e assuma a seguinte condição.

Condição de Milnor: Suponha que em alguma vizinhança aberta $U$ da origem $0 \in \mathbb{R}^{n}$, tem-se $\sum(f):=\{x \in U \mid \operatorname{rank} J f(x)$ não é maximal $\}=\{0\}$, ou seja, 0 é um ponto singular isolado de $f$, onde $J f(x)$ denota o Jacobiano de $f$ em $x$.

\footnotetext{
${ }^{1}$ o fibrado tangente $T F_{\theta}$ é trivial.
} 
Milnor mostrou que, desta forma, existe $\varepsilon_{0}>0$ suficientemente pequeno tal que para $0<\varepsilon \leqslant \varepsilon_{0}$ podemos encontrar $\delta$, com $0<\delta \ll \varepsilon$ de modo que a aplicação

$$
f_{\mid}: f^{-1}\left(S_{\delta}^{p-1}\right) \cap B_{\varepsilon}^{n} \rightarrow S_{\delta}^{p-1}
$$

é uma projeção suave de um fibrado localmente trivial onde $S_{\delta}^{p-1}$ é a esfera $(p-1)$ dimensional de raio $\delta$, centrada na origem e $B_{\varepsilon}^{n}$ é a bola fechada de dimensão $n$ e raio $\varepsilon$ centrada na origem. Usando campo de vetores ele construiu um difeomorfismo em $B_{\varepsilon}^{n} \backslash\{f=0\}$ que leva o espaço total $f^{-1}\left(S_{\delta}^{p-1}\right) \cap B_{\varepsilon}^{n}$ da fibração (0.0.2) no complementar $S_{\varepsilon}^{n-1} \backslash \operatorname{Int} N\left(K_{\varepsilon}\right)$ de uma vizinhança tubular aberta de $K_{\varepsilon}=f^{-1}(0) \cap S_{\varepsilon}^{n-1}$, obtendo uma aplicação

$$
S_{\varepsilon}^{n-1} \backslash \operatorname{IntN}\left(K_{\varepsilon}\right) \rightarrow S_{\delta}^{p-1}
$$

que também é um fibrado suave localmente trivial cuja fibra $F_{f}$ é uma variedade de dimensão $(n-p)$, suave, compacta e com bordo difeomorfo a $K_{\varepsilon}$. Se $K_{\varepsilon}$ não for vazio, temos que sempre podemos estender a fibração (0.0.3) sobre $N\left(K_{\varepsilon}\right) \backslash K_{\varepsilon}$ e escalonar para a esfera unitária de modo a obter um fibrado suave localmente trivial

$$
S_{\varepsilon}^{n-1} \backslash K_{\varepsilon} \rightarrow S^{p-1}
$$

cuja fibra é o interior de uma variedade compacta com bordo $K_{\varepsilon}$.

Milnor também observou que, em geral, tanto a projeção da fibração $S_{\varepsilon}^{n-1} \backslash \operatorname{Int} N\left(K_{\varepsilon}\right) \rightarrow$ $S^{p-1}$ quanto a projeção (0.0.4) não são dadas por $\frac{f}{\|f\|}$. No entanto, o mesmo não apresentou condições necessárias e suficientes para garantir que a projeção da fibração (0.0.4) fosse dada por $\frac{f}{\|f\|}$.

No final da década de 80, A. Jacquemard apresentou em [26, 27] uma caracterização dos pontos críticos da projeção $\frac{f}{\|f\|}: S_{\varepsilon}^{n-1} \backslash K_{\varepsilon} \rightarrow S^{1}$ como segue

Teorema 0.0.1. [26, 27] Considere $f=(P, Q): U \subset \mathbb{R}^{n} \rightarrow \mathbb{R}^{2}$ uma aplicação analítica com singularidade isolada na origem. Um ponto $x \in S_{\varepsilon}^{n-1}$ é crítico da projeção $\frac{f}{\|f\|}$ se, e somente se, existe $\lambda \in \mathbb{R}$ tal que $P(x) \nabla Q(x)-Q(x) \nabla P(x)=\lambda x$.

A prova do teorema apresentada nas referências não é tão simples pois utiliza bastante técnica e ferramentas não usuais de conjuntos semi-algébricos. No entanto, uma prova mais elementar foi feita por Araújo dos Santos em [1].

O estudo de singularidades de aplicações analíticas reais $f$ cuja aplicação $\frac{f}{\|f\|}: S_{\varepsilon}^{n-1} \backslash K_{\varepsilon} \rightarrow S^{p-1}$ é a projeção de um fibrado suave localmente trivial tem sido desenvolvido por vários matemáticos (ver, por exemplo, [44] para uma introdução ao 
tema). Em [43], os autores M. Ruas, J. Seade e A. Verjovsky introduziram a condição de Milnor forte na origem, a qual de forma mais direta significa que um germe de aplicação $f:\left(\mathbb{R}^{n}, 0\right) \rightarrow\left(\mathbb{R}^{2}, 0\right)$, satisfazendo a condição de Milnor, tem a projeção do fibrado (0.0.4) dada por $\frac{f}{\|f\|}$.

Na direção de singularidades não-isoladas, usando um importante conceito introduzido por E. Looijenga, o de NS-par, os autores R. Araújo dos Santos e M. Tibăr consideraram germes de aplicações analíticas reais $f:\left(\mathbb{R}^{n}, 0\right) \rightarrow\left(\mathbb{R}^{p}, 0\right)$ e introduziram uma condição que além de garantir que a aplicação projeção do fibrado associado é dada por $\frac{f}{\|f\|}$, também estende a noção de NS-par ou estrutura open book superior da esfera $S_{\varepsilon}^{n-1}$ (vide [6]).

Ainda no caso real, notamos que em [39], Milnor apresentou alguns resultados sobre a topologia da fibra. Ele mostrou que se $n<2(p-1)$, a fibra é necessariamente contrátil e na demonstração deste resultado, encontramos um outro que também é muito importante e vale para todo $n>p$. Tal resultado afirma que se o link $K_{\varepsilon}$ for não vazio, então a fibra é $(p-2)$-conexa. Uma questão natural é verificar a existência de um Teorema do Buquê para o caso real. Mas, em geral, não é possível garantir tal existência, uma vez que, através de construções devidas a Zeeman e Looijenga, podemos obter exemplos de fibras de Milnor cujos grupos fundamentais não são livres. No entanto, neste trabalho, encontramos condições nas quais garantimos que a fibra tem o tipo de homotopia de um buquê de esferas em vários pares de dimensões $(n, p), n>p \geqslant 2$. Mais detalhes são apresentados no último capítulo.

Objetivando apresentar resultados e ferramentas necessários para o entendimento dos resultados principais, estruturamos o texto da seguinte forma. No capítulo 1, apresentamos conceitos básicos e resultados clássicos da Topologia Algébrica e da Topologia Diferencial, além de um breve estudo dos Grupos de Lie.

No Capítulo 2, fazemos um estudo da existência da fibração de Milnor para germes de aplicações analíticas reais com conjunto singular qualquer, utilizando as condições $(a)$ e (b) de Milnor, as quais foram definidas por D. Massey em [35]. Apresentamos alguns avanços encontrados na literatura a respeito dos questionamentos feitos por Milnor no livro "Singular point of complex hypersurfaces". Além disso, apresentamos neste capítulo a ideia de algumas construções feitas por Emil Artin, Erik Zeeman e Eduard Looijenga, as quais fornecem condições para enunciarmos e provarmos novos resultados.

Finalizados os dois primeiros capítulos que reúnem importantes informações para o desenvolvimento desta tese, estamos em condições de começar a apresentar resultados bem recentes e o conteúdo novo deste trabalho. Assim, o objetivo do Capítulo 3 é relem- 
Introdução

brar alguns resultados por completitude. Dessa forma, apresentaremos um refinamento de um teorema provado por Church e Lamotke (Teorema 2.3.4), o qual responde uma das questões de J. Milnor acerca da não-trivialidade de um germe de aplicação polinomial real com singularidade isolada. Nosso resultado, Teorema 3.0.3, cobre todos os casos da fibra de Milnor 3-dimensional, caracterizando aplicações triviais usando conceitos mais "manejáveis" como conexidade, característica de Euler e grau de Brouwer de uma aplicação.

No Capítulo 4, objetivamos caracterizar NS-pares $\left(S^{5}, K\right) \operatorname{com} \operatorname{dim} K=2$, e usar tal caracterização para provar a existência de germes de aplicações polinomiais reais nãotriviais $\left(\mathbb{R}^{6}, 0\right) \rightarrow\left(\mathbb{R}^{3}, 0\right)$ com uma singularidade isolada na origem, respondendo o Problema 2.3.3 proposto por Milnor, que, segundo nossas pesquisas, se encontrava em aberto até o momento. Para isso, utilizamos ferramentas de espaços de configuração e um resultado de L. Funar provado em [20, Seção 2.7], o qual está enunciado na Seção 2.5 do Capítulo 2. Mais precisamente, primeiro classificaremos fibrados $E^{5} \rightarrow S^{2}$ cuja fibra é a 3-esfera com os interiores de uma união disjunta de 3-discos removidos, e que satisfazem a condição que as fibrações restritas ao bordo são triviais. Mostraremos (Lema 4.2.1) que as classes de isomorfismo de tais fibrados estão em correspondência 1 - 1 com o segundo grupo de homotopia de um certo espaço de configuração, cujas classes correspondem a matrizes de inteiros anti-simétricas. Então, concluiremos que um dado fibrado $E^{5} \rightarrow S^{2}$ está associado a um NS-par $\left(S^{5}, K\right)$ se, e somente se, a matriz anti-simétrica é unimodular (ver o principal Teorema 4.2.3). Como uma consequência, veremos que o número de componentes de bordo de uma fibra é sempre ímpar. Além disso, isso nos permitirá construir muitos NS-pares $\left(S^{5}, K\right)$ não-triviais, e então a construção de E. Looijenga [34] (ou veja a Seção 2.4 do Capítulo 2) conduzirá para germes de aplicações polinomiais não-triviais com uma singularidade isolada. Na Seção 4.3, faremos uma generalização de alguns resultados da Seção 4.2, objetivando obter novos exemplos não-triviais nos pares de dimensões $(n, p)$ com $n-p \geqslant 4$. Tais resultados também estendem os de Church e Lamotke em [12], pois no nosso caso a fibra não é contrátil.

Com respeito ao estudo da topologia da fibra de Milnor real, podemos citar [4], [14], [15], [16]. Dando continuidade a esses estudos, introduzimos condições suficientes sob as quais a fibra de Milnor nos pares de dimensões $(2 n, n)$ e $(2 n+1, n)$, é, a menos de homotopia, um buquê de esferas. Isto é feito no Capítulo 5 , onde os principais resultados são as Proposições 5.3.1 e 5.3.4. Mais precisamente, provamos que a fibra real nos pares de dimensões $(2 n, n), n \geqslant 2$ e $(2 n+1, n), n \geqslant 3$ tem, respectivamente, o tipo de homotopia de um buquê de $(n-1)$-esferas e o tipo de homotopia de um buquê de $n$ - e $(n-1)$-esferas. Além disso, como aplicações, garantimos a existência de exemplos de germes de aplicações polinomiais em todos os pares de dimensões $(2 n, p)$ e $(2 n+1, p), 2 \leqslant p \leqslant n$, tais que a fibra de Milnor tem essas características. 


\section{Preliminares}

Neste capítulo relembramos algumas definições e resultados clássicos necessários para a compreensão dos capítulos subsequentes. No entanto, advertimos o leitor que existem outros resultados que usaremos ao longo do texto, mas nos limitaremos a informá-los no momento conveniente.

Em todo o trabalho, os grupos de (co)homologia são com coeficientes inteiros a menos

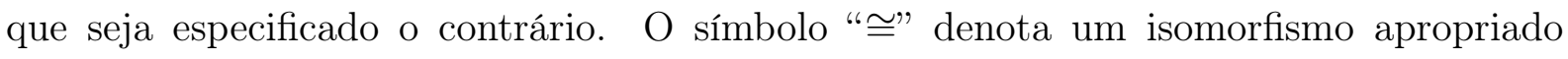
entre objetos algébricos, " $\approx$ " um difeomorfismo entre variedades suaves e " $\simeq$ " denota uma equivalência de homotopia. Além disso, estamos considerando uma $n$-esfera como a esfera padrão $S^{n}$.

\subsection{Ferramentas da Topologia Algébrica}

Considere $X$ um espaço topológico, $A$ um subespaço de $X$ e as seguintes notações:

1. $\pi_{n}(X)$ é o $n$-ésimo grupo de homotopia de $X$;

2. $H_{n}(X)$ (resp. $\left.H_{n}(X, A)\right)$ é o $n$-ésimo grupo de homologia singular de $X$ (resp. do $\operatorname{par}(X, A))$;

3. $H^{n}(X)$ (resp. $\left.H^{n}(X, A)\right)$ é o $n$-ésimo grupo de cohomologia singular de $X$ (resp. do $\operatorname{par}(X, A))$.

Observação 1.1.1. Os grupos de homologia reduzida de um espaço topológico $X$, denotados por $\tilde{H}_{n}(X)$, se relacionam com os grupos de homologia singular da seguinte forma:

$$
H_{n}(X) \cong \begin{cases}\tilde{H}_{n}(X), & n>0 \\ \tilde{H}_{0}(X) \oplus \mathbb{Z}, & n=0\end{cases}
$$

O resultado a seguir descreve quando os grupos relativos $H_{n}(X, A)$ não são afetados ao extrair um subconjunto $Z \subset A$ e sua prova pode ser encontrada em [24], página 124 . 
Teorema 1.1.2. (Teorema de Excisão) Seja $(X, A)$ um par de espaços topológicos e $Z \subset$ $A \subset X$ tal que $\bar{Z} \subset \AA$. Então a inclusão $i:(X-Z, A-Z) \hookrightarrow(X, A)$ induz isomorfismo $i_{*}: H_{p}(X-Z, A-Z) \rightarrow H_{p}(X, A), \forall p \geqslant 0$. Equivalentemente, para subespaços $A, B \subset X$ cujos interiores cobrem $X$, a inclusão $(B, A \cap B) \hookrightarrow(X, A)$ induz isomorfismos $H_{p}(B, A \cap$ $B) \rightarrow H_{p}(X, A)$ para todo $p$.

Proposição 1.1.3. Se $M$ é uma variedade topológica n-dimensional compacta, conexa e orientável, com bordo não-vazio, então $H_{i}(M)=0$ para $i \geqslant n$.

Apresentamos a seguir, um resultado básico na estrutura dos grupos de homologia e de cohomologia de variedades.

Teorema 1.1.4. (Dualidade de Poincaré - [48, p.297]) Se U é uma orientação de uma $n$-variedade compacta $X$, então para todo q existe um isomorfismo

$$
H_{q}(X) \cong H^{n-q}(X) .
$$

Com o intuito de relacionar a estrutura de um subconjunto da esfera com o seu complemento, apresentamos a seguir o Teorema da dualidade de Alexander.

Teorema 1.1.5. (Dualidade de Alexander - [24, p.254]) Se K é um subespaço próprio de $S^{n}$, não-vazio, compacto e localmente contrátil, então para todo i tem-se

$$
\tilde{H}_{i}\left(S^{n} \backslash K\right) \cong \tilde{H}^{n-i-1}(K) .
$$

Uma aplicação contínua $f: X \rightarrow Y$ é chamada uma equivalência de homotopia se existe uma aplicação contínua $g: Y \rightarrow X$ tal que $f \circ g \simeq I d_{Y}$ e $g \circ f \simeq I d_{X}$. Neste caso, a aplicação $g$ é chamada uma inversa homotópica de $f$ e dizemos que os espaços $X$ e $Y$ são equivalentes homotópicos ou, em outras palavras, que $X$ e $Y$ têm o mesmo tipo de homotopia e usamos a notação $X \simeq Y$.

Dizemos que a aplicação contínua $f: X \rightarrow Y$ é uma equivalência de homotopia fraca se ela induz isomorfismos $\pi_{n}\left(X, x_{0}\right) \rightarrow \pi_{n}\left(Y, f\left(x_{0}\right)\right)$ para todo $n \geqslant 0$ e todas as escolhas de ponto base $x_{0}$.

Considere os espaços topológicos $X$ e $A \subset X$. Dizemos que $A$ é um retrato de $X$ se a aplicação $I d_{A}: A \rightarrow A$ se estende a uma função contínua $r: X \rightarrow A$, isto é, a composição $A \stackrel{i}{\rightarrow} X \stackrel{r}{\rightarrow} A$, onde $i$ é a aplicação inclusão, corresponde à aplicação $I d_{A}$. A função $r$ é chamada retração. Se, além disso, a composição $X \stackrel{r}{\rightarrow} A \stackrel{i}{\rightarrow} X$ é homotópica à $I d_{X}$, dizemos que $A$ é um retrato por deformação de $X$.

Definição 1.1.6. Dado $k \geqslant 0$ inteiro, dizemos que um espaço topológico $X$ é $k$-conexo se é conexo por caminhos e $\pi_{i}(X)=0$ para $0 \leqslant i \leqslant k$. 
Teorema 1.1.7. (Teorema do Isomorfismo de Hurewicz - [49, p.185]) Se um espaço Xé $(n-1)$-conexo, $n \geqslant 2$, então $h: \pi_{q}\left(X, x_{0}\right) \rightarrow \widetilde{H}_{q}(X)$ é um isomorfismo para $q \leqslant n$ e um epimorfismo para $q=n+1$. No caso $n=1$, $h$ é um epimorfismo para $q=n$.

Observação 1.1.8. Nas hipóteses do Teorema 1.1.7, quando $n=1=q$, o núcleo da aplicação $h: \pi_{1}\left(X, x_{0}\right) \rightarrow \widetilde{H}_{1}(X)$ é o subgrupo comutador de $\pi_{1}(X)$. Então $h$ induz um isomorfismo entre a abelianização de $\pi_{1}(X)$ e o grupo $H_{1}(X)$. Para detalhes, ver [24, Teorema 2A.1].

Apresentaremos, a seguir, duas versões para o Teorema de Whitehead (que é uma consequência do Teorema 1.1.7), sendo que a segunda é válida quando assumimos que os espaços são CW-complexos.

Teorema 1.1.9. (Teorema de Whitehead - [48, p.399]) Sejam X e Y espaços 0-conexos (ambos com ponto base) e seja $f:\left(X, x_{0}\right) \rightarrow\left(Y, y_{0}\right)$ uma aplicação. Se existe $n \geqslant 1$ tal que

$$
f_{\sharp}: \pi_{q}\left(X, x_{0}\right) \rightarrow \pi_{q}\left(Y, y_{0}\right)
$$

é um isomorfismo para $q<n$ e um epimorfismo para $q=n$, então

$$
f_{*}: H_{q}\left(X, x_{0}\right) \rightarrow H_{q}\left(Y, y_{0}\right)
$$

é um isomorfismo para $q<n$ e um epimorfismo para $q=n$. Por outro lado, se $X$ e $Y$ são 1 -conexos e $f_{*}$ é um isomorfismo para $q<n$ e um epimorfismo para $q=n$, então $f_{\sharp}$ é um isomorfismo para $q<n$ e um epimorfismo para $q=n$.

Teorema 1.1.10. ([24, Teorema 4.5]) Se uma aplicação $f: X \rightarrow Y$ entre $C W$ complexos conexos induz isomorfismos $f_{\sharp}: \pi_{n}(X) \rightarrow \pi_{n}(Y)$ para todo $n$, então $f$ é uma equivalência de homotopia. No caso de $f$ ser a inclusão de um subcomplexo $X \hookrightarrow Y$, a conclusão é mais forte: $X$ é um retrato por deformação de $Y$.

Teorema 1.1.11. ([48, Teorema 7, p.43]) Uma aplicação continua $\alpha: S^{n} \rightarrow X$ representa um elemento trivial de $\pi_{n}(X)$ para $n \geqslant 1$ se, e somente se, $\alpha$ pode ser continuamente estendida para $D^{n+1} \rightarrow X$.

O resultado a seguir caracteriza o subgrupo de torção $(n-1)$-dimensional de uma $n$-variedade conexa e será muito importante para o desenvolvimento do Capítulo 5.

Lema 1.1.12. ([8, Corolário 7.13]) Se $M$ é uma variedade conexa, então o subgrupo de torção $T H_{n-1}(M)$ é $\mathbb{Z}_{2}$ se $M$ é compacta e não-orientável e é 0 se $M$ é não-compacta ou orientável. 
A partir de agora, utilizaremos a seguinte notação: dada $M$ variedade com bordo, denotaremos o bordo de $M$ por $\partial M$. Quando $M$ for uma variedade compacta com $\partial M=\emptyset$, diremos que $M$ é uma variedade fechada.

Definição 1.1.13. A variedade $n$-dimensional $M$ é uma $n$-esfera de homologia se $M$ é fechada e tem a homologia da esfera $S^{n}$.

Quando $M$ é equivalente homotópica à esfera $S^{n}$, dizemos que $M$ é uma $n$-esfera de homotopia. Note que toda esfera de homotopia é uma esfera de homologia.

A seguir veremos duas operações entre espaços. Tais operações serão utilizadas nos dois últimos capítulos deste trabalho.

Wedge. Considere os espaços $X$ e $Y$ com os respectivos pontos escolhidos $x_{0}$ e $y_{0}$. Chamaremos de wedge, e denotaremos por $X \vee Y$, o quociente da união disjunta $X \dot{\cup} Y$ obtido pela identificação de $x_{0}$ e $y_{0}$ a um único ponto. Por exemplo, $S^{1} \vee S^{1}$ é homeomorfo à "figura 8", dois círculos que se intersectam em um ponto. Um wedge de esferas arbitrárias é frequentemente chamado de buquê de esferas.

Soma conexa. Considere duas variedades disjuntas $n$-dimensionais conexas $M_{1}$ e $M_{2}$, e o disco unitário $n$-dimensional $D^{n}$. Escolha mergulhos $i_{1}: D^{n} \rightarrow M_{1}$ e $i_{2}: D^{n} \rightarrow M_{2}$ de modo que $i_{1}$ preserva orientação e $i_{2}$ inverte orientação. A soma conexa de $M_{1}$ e $M_{2}$, denotada por $M_{1} \sharp M_{2}$, é obtida da soma disjunta $\left(M_{1}-i_{1}(0)\right)+\left(M_{2}-i_{2}(0)\right)$ por identificação de $i_{1}(t u)$ com $i_{2}((1-t) u)$, para cada vetor unitário $u \in S^{n-1}$ e cada $0<t<1$. A orientação para $M_{1} \sharp M_{2}$ deve ser escolhida de forma compatível com a orientação de $M_{1}$ e de $M_{2}$.

Característica de Euler. Para um $C W$ complexo finito $X$, a característica de Euler $\chi(X)$ é definida como sendo a soma alternada $\sum_{n}(-1)^{n} c_{n}$, onde $c_{n}$ é o número de $n$ células de $X$, generalizando a fórmula familiar vértices - arestas + faces para complexos 2-dimensionais. A seguir, veremos que $\chi(X)$ pode ser definida apenas em termos de homologia. Em particular, $\chi(X)$ não depende da escolha da estrutura de $C W$ sobre $X$.

Teorema 1.1.14. [24, Teorema 2.44, p.146] $\chi(X)=\sum_{n}(-1)^{n} \operatorname{rank} H_{n}(X)$.

Aqui, rank corresponde ao posto de $H_{n}(X)$ como um grupo abeliano finitamente gerado.

\subsubsection{Colagem de Espaços}

Dados $X, Y$ espaços topológicos e $A \subset X$ fechado tais que $X \cap Y=\emptyset$ e $f: A \rightarrow Y$ uma função contínua, veremos como construir um novo espaço topológico denotado por $X \cup_{f} Y$ e chamado o espaço obtido por colagem dos espaços $X$ e $Y$ via a aplicação $f: A \rightarrow Y$. 
Definição 1.1.15. A união livre ou soma topológica de dois espaços topológicos $(X, \tau)$, $\left(Y, \tau^{\prime}\right)$ tal que $X \cap Y=\emptyset$ é o conjunto $X \dot{\cup} Y$ munido da topologia

$$
\tau_{X \dot{\cup} Y}:=\left\{U \subset X \dot{\cup} Y ; U \cap X \in \tau \text { e } U \cap Y \in \tau^{\prime}\right\}
$$

Observações 1.1.16. 1) O espaço topológico $\left(X \cup \dot{\cup} Y, \tau_{X \cup Y}\right)$ será denotado por $X+Y$.

2) $X \cap X=X \in \tau$ e $X \cap Y=\emptyset \in \tau^{\prime} \Rightarrow X \in \tau_{X \dot{Y} Y}(X$ é aberto em $X+Y)$. Analogamente, $Y$ é aberto em $X+Y$. Ou seja, $X$ e $Y$ são abertos disjuntos em $X+Y$.

3) Todo aberto na topologia de $X$ (resp. de $Y$ ) é aberto na topologia de $X+Y$.

Definição 1.1.17. Sejam $X$ e $Y$ espaços topológicos e $A \subset X$ fechado. Seja $f: A \rightarrow Y$ uma aplicação contínua. Denotamos por $X \cup_{f} Y$ o espaço quociente de $X+Y$ pela relação de equivalência $\sim$ definida da seguinte forma: para $u, v \in X+Y$, temos $u \sim v$ se, e somente se, pelo menos uma das seguintes condições é satisfeita:

1. $u=v$;

2. $u, v \in A$ e $f(u)=f(v)$;

3. $u \in A$ e $v=f(u) \in Y$.

O espaço $X \cup_{f} Y$ é chamado de espaço colagem de $X$ a $Y$ via a aplicação $f$, e a aplicação $f$ é chamada de aplicação colagem.

Observação 1.1.18. $X \cup_{f} Y$ é munido da topologia quociente coinduzida pela projeção natural $p: X+Y \rightarrow X \cup_{f} Y$, ou seja, $U \subset X \cup_{f} Y$ é aberto $\Leftrightarrow p^{-1}(U)$ é aberto em $X+Y$.

\subsection{Ferramentas da Topologia Diferencial}

Nesta seção, veremos resultados envolvendo variedades e aplicações suaves (de classe $\left.C^{\infty}\right)$.

\subsubsection{Fibrados e Fibrações}

Definição 1.2.1. Sejam $M$ e $N$ variedades suaves, com $\partial M$ não-vazio. Uma aplicação $f: M \rightarrow N$ é uma submersão suave se as restrições $f: M \backslash \partial M \rightarrow N$ e $f: \partial M \rightarrow N$ forem ambas submersões. 
Definição 1.2.2. Um fibrado suave localmente trivial é uma quádrupla $(E, p, B, F)$, onde $E, B, F$ são variedades suaves, $B$ é conexo e $p: E \rightarrow B$ é uma submersão suave sobrejetiva, satisfazendo as seguintes condições: para cada $y \in B$ existe uma vizinhança $V_{y}$ de $y$ em $B$ e um difeomorfismo $h: p^{-1}\left(V_{y}\right) \rightarrow V_{y} \times F$ tal que $p=\pi_{1} \circ h$, ou seja, o diagrama abaixo é comutativo:

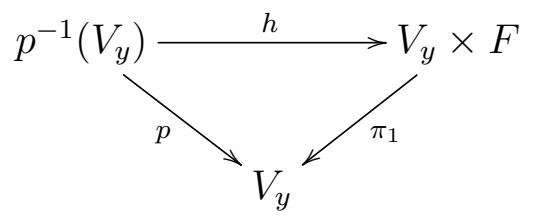

Aqui, $\pi_{1}: V_{y} \times F \rightarrow V_{y}$ é a projeção na primeira coordenada.

A variedade $E$ é chamada de espaço fibrado ou espaço total. B é chamado de espaço base, ou simplesmente base. A variedade $F$ é a fibra e a aplicação $p$ é chamada de aplicação fibrada ou projeção.

Quando podemos escolher $V_{y}=B$, dizemos que o fibrado é trivial e desta forma, $E$ é difeomorfo a $B \times F$.

Segue da definição acima que para cada $y \in B$, a variedade $p^{-1}(y)$ é difeomorfa a $F$ e é chamada de fibra sobre o ponto y de $B$.

A definição a seguir pode ser encontrada em [13, Capítulo 3].

Definição 1.2.3. (Fibra equivalente) Duas aplicações fibradas suaves (resp. topológicas) $p: E \rightarrow B$ e $p^{\prime}: E^{\prime} \rightarrow B$ são ditas serem fibras equivalentes difeomorfas (resp. fibras equivalentes homotópicas) se existe um difeomorfismo (resp. aplicação contínua) $h: E \rightarrow$ $E^{\prime}$ tal que:

(i) $p^{\prime} \circ h=p$;

(ii) para todo $b \in B, h$ induz uma aplicação $h_{b}: p^{-1}(b) \rightarrow p^{-1}(b)$ que é um difeomorfismo (resp. uma equivalência de homotopia).

Sejam $\xi=(E, p, B, F)$ um fibrado e $f: B^{\prime} \rightarrow B$ uma aplicação contínua. O fibrado induzido de $\xi$ por $f$, o qual denotamos por $f^{*}(\xi)$, tem como espaço base $B^{\prime}$ e seu espaço total é definido como sendo o subespaço

$$
f^{*}(E)=\left\{\left(b^{\prime}, x\right) \in B^{\prime} \times E: f\left(b^{\prime}\right)=p(x)\right\} .
$$

A aplicação fibrada é dada pela projeção

$$
p^{\prime}: f^{*}(E) \rightarrow B^{\prime}, p^{\prime}\left(b^{\prime}, x\right)=b^{\prime}
$$


enquanto a aplicação

$$
f^{\prime}: f^{*}(E) \rightarrow E
$$

leva a fibra de $f^{*}(E)$ sobre cada ponto $b^{\prime} \in B^{\prime}$ isomorficamente na fibra de $E$ sobre $f\left(b^{\prime}\right)$. O fibrado induzido de $\xi$ por $f, f^{*}(\xi)=\left(f^{*}(E), p^{\prime}, B^{\prime}, F\right)$ também é chamado o pullback de $\xi$ por $f$. Em resumo, temos o seguinte diagrama comutativo.

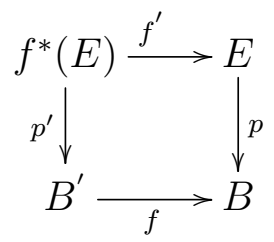

Além disso, o pullback de um fibrado trivial é um fibrado trivial (vide [25]).

Os fibrados vetoriais formam uma classe especial de fibrados cujas fibras são espaços vetoriais. De forma mais precisa, um fibrado vetorial real de posto $n$ é um fibrado $\xi=$ $\left(E, p, B, \mathbb{R}^{n}\right)$ tal que para cada $b \in B, p^{-1}(b)$ possui uma estrutura de $\mathbb{R}$-espaço vetorial $n$-dimensional e para uma trivialização local qualquer $\left(U_{\alpha}, \varphi_{\alpha}\right)$ de $\xi$, as restrições

$$
\varphi_{\alpha \mid p^{-1}(b)}: p^{-1}(b) \rightarrow\{b\} \times \mathbb{R}^{n}
$$

são isomorfismos de espaços vetoriais, para cada $b \in U_{\alpha}$.

A quádrupla $\left(T M, \pi, M, \mathbb{R}^{n}\right)$ é um fibrado suave localmente trivial conhecido como fibrado tangente da variedade suave $n$-dimensional $M$ e além disso é um exemplo de fibrado vetorial de posto $n$ cujo espaço total é a união disjunta dos espaços tangentes em todos os pontos de $M$, isto é,

$$
T M=\coprod_{p \in M} T_{p} M=\bigcup_{p \in M}\left(\{p\} \times T_{p} M\right),
$$

onde $T_{p} M$ é o espaço tangente à $M$ no ponto $p$. A projeção do fibrado tangente, $\pi: T M \rightarrow$ $M$, envia cada vetor $v$ em $T_{p} M$ para o ponto $p$ no qual ele é tangente, isto é, $\pi(p, v)=p$.

Definição 1.2.4. Dizemos que $M$ é paralelizável quando a quádrupla $\left(T M, \pi, M, \mathbb{R}^{n}\right)$ é um fibrado suave trivial.

Considere $Z$ uma subvariedade $n$-dimensional da variedade Riemanniana $m$-dimensional $M$. O espaço normal a $Z$ em um ponto $p \in Z$, denotado por $N_{p} Z$, é o subespaço de $T_{p} M$ consistindo de todos os vetores $v$ que são ortogonais a $T_{p} Z$. O fibrado normal de $Z$ em $M$ é definido como sendo o fibrado vetorial de posto $(m-n)$ cujo espaço total é a união disjunta dos espaços normais em todos os pontos de $Z$ :

$$
N Z:=\coprod_{p \in Z} N_{p} Z:=\left\{(p, v): p \in Z, v \in T_{p} M \text { e } v \perp T_{p} Z\right\} .
$$


Definição 1.2.5. Dado um fibrado suave localmente trivial $(X, p, B, F)$, uma seção do fibrado é uma aplicação suave $f: B \rightarrow X$ tal que $p \circ f(b)=b$ para todo $b \in B$.

Decorre da definição que uma seção de um fibrado é uma aplicação injetiva.

Definição 1.2.6. Seja $M$ uma variedade suave. Um campo de vetores $v$ em $M$ é uma aplicação suave $v: M \rightarrow T M$ tal que $\pi \circ v$ é a aplicação identidade, onde $\pi: T M \rightarrow M$ é a projeção natural.

Observações 1.2.7. (1) De acordo com a definição 1.2.5, um campo de vetores suave tangente à variedade $M$ é uma seção suave da quádrupla $\left(T M, \pi, M, \mathbb{R}^{n}\right)$.

(2) Se $M \subset \mathbb{R}^{n}$, então dizemos que um campo de vetores é tangente à $M$ quando a aplicação suave $v: M \rightarrow \mathbb{R}^{n}$ é tal que para todo $x \in M, v(x) \in T_{x} M$.

Um $k$-referencial sobre uma variedade suave $n$-dimensional $M, 1 \leqslant k \leqslant n$, é uma $k$-upla $v=\left(v_{1}, v_{2}, \ldots, v_{k}\right)$ de seções linearmente independentes do fibrado tangente de $M$, ou seja, é uma $k$-upla em que cada $v_{i}, 1 \leqslant i \leqslant k$, é um campo de vetores tangentes à $M$ e o conjunto formado pelos $k$ vetores $v_{i}(x)$ é linearmente independente para cada $x \in M$.

Agora considere o conjunto de todos os $k$-referenciais ortonormais em $\mathbb{R}^{n}$, isto é, $k$ uplas de vetores ortonormais. Tal conjunto é chamado variedade de Stiefel e será denotado por $V_{k}\left(\mathbb{R}^{n}\right)$. Esta variedade pode ser pensada como o conjunto de matrizes $n \times k$, escrevendo um $k$-referencial como uma matriz de $k$ vetores-colunas em $\mathbb{R}^{n}$. A condição de ortonormalidade é expressa por $A^{T} A=I$, onde $A^{T}$ denota a matriz transposta de $A$ e $I$ denota a matriz identidade $k \times k$. Como casos especiais, temos $V_{n}\left(\mathbb{R}^{n}\right)=O(n)$ (grupo ortogonal) e $V_{1}\left(\mathbb{R}^{n}\right)=S^{n-1}$.

A seguir, veremos a definição de um fibrado associado ao fibrado tangente de uma variedade.

Definição 1.2.8. Seja $\pi: T M \rightarrow M$ a projeção do fibrado tangente da variedade $n$ dimensional $M$. Para cada $y \in M$, podemos considerar a variedade de Stiefel $V_{k}\left(T_{y} M\right)$, que consiste de todas as $k$-uplas $\left(v_{1}, v_{2}, \ldots, v_{k}\right)$ de vetores linearmente independentes do espaço tangente $T_{y} M$. O fibrado cujas fibras são as variedades de Stiefel $V_{k}\left(T_{y} M\right), y \in M$, cujo espaço total, denotado por $V_{k}(T M)$, é a união destas fibras e que é associado ao fibrado tangente é chamado fibrado de Stiefel. A aplicação $p: V_{k}(T M) \rightarrow M$ é a projeção que associa a cada $k$-referencial $\left(v_{1}, v_{2}, \ldots, v_{k}\right) \in V_{k}\left(T_{y} M\right)$ o ponto $y \in M$.

Observações 1.2.9. (1) Note que a fibra do fibrado de Stiefel é, na verdade, $V_{k}\left(\mathbb{R}^{n}\right)$. De fato, observe que, para cada $y \in M$ temos que $T_{y} M$ é isomorfo a $\mathbb{R}^{n}$. 
(2) No caso particular de $M=S^{3}$, temos que $\pi: T S^{3} \rightarrow S^{3}$ é um fibrado trivial. Fixando a sua trivialização, podemos considerar a projeção do fibrado de Stiefel $p: V_{3}\left(T S^{3}\right) \rightarrow S^{3}$, cuja fibra, neste caso, corresponde ao grupo ortogonal $O(3)$.

(3) Quando um fibrado tangente é trivial, o mesmo ocorre com o fibrado de Stiefel associado.

O resultado a seguir é uma condição suficiente para uma aplicação entre duas variedades ser a projeção de um fibrado suave localmente trivial e será enunciado para variedades com bordo uma vez que a situação mais geral neste trabalho é a utilização de variedades desta natureza. O enunciado para variedades sem bordo pode ser encontrado em [44].

Teorema 1.2.10. (Teorema de Ehresmann) Sejam $M, N$ variedades suaves com $N$ conexa, $M$ com bordo (o qual pode ser vazio) e $f: M \rightarrow N$ uma submersão suave, sobrejetora e própria. Então a quádrupla $\left(M, f, N, f^{-1}(y)\right)$ é um fibrado suave localmente trivial, onde $y \in N$ é um ponto fixo.

A seguir, temos uma relação entre fibração e teoria de homotopia (ver [24, p.376]).

Teorema 1.2.11. Seja $(E, p, B, F)$ um fibrado suave. Então existe a seguinte sequência (longa) exata

$$
\cdots \rightarrow \pi_{n}(F) \rightarrow \pi_{n}(E) \rightarrow \pi_{n}(B) \rightarrow \pi_{n-1}(F) \rightarrow \cdots \rightarrow \pi_{1}(B) \rightarrow \pi_{0}(F) \rightarrow \pi_{0}(E) \rightarrow 0 .
$$

O resultado a seguir relaciona fibração e teoria de homologia/cohomologia. Ele pode ser encontrado, por exemplo, em [48, p.456].

Proposição 1.2.12. (Sequências de Wang) Seja $p: E \rightarrow S^{n}, n>1$, uma fibração com fibra F. Existem sequências exatas:

$$
\begin{gathered}
\cdots \rightarrow H_{q}(F) \rightarrow H_{q}(E) \rightarrow H_{q-n}(F) \rightarrow H_{q-1}(F) \rightarrow H_{q-1}(E) \rightarrow H_{q-n-1}(F) \rightarrow \cdots \\
\cdots \rightarrow H^{q}(E) \rightarrow H^{q}(F) \rightarrow H^{q-n+1}(F) \rightarrow H^{q+1}(E) \rightarrow H^{q+1}(F) \rightarrow H^{q-n+2}(F) \rightarrow \cdots .
\end{gathered}
$$

Observação 1.2 .13 (Importante). Sabendo que todo fibrado cujo espaço base é uma variedade é chamado uma fibração, a qual é uma função contínua que tem a propriedade do levantamento de homotopia com relação a qualquer espaço topológico X, muitas vezes vamos nos referir à projeção $p: E \rightarrow B$ simplesmente por fibração suave. Além disso, se a fibra $F$ for conhecida, chamaremos a quádrupla $(E, p, B, F)$ de $F$-fibração. Vale lembrar que nosso objetivo principal é fazer o estudo da fibração de Milnor. No entanto, advertimos o leitor que, no Capítulo 4, trabalharemos com uma classe especial de fibrado cujos espaços não são variedades, mas que será chamado fibração localmente trivial. O motivo se deve ao fato de utilizarmos alguns resultados de Cerf-Palais. Neste caso, de acordo com [11, 
p.114], se $E$ e $B$ são dois espaços topológicos e $p: E \rightarrow B$ é uma aplicação contínua, $p$ é chamada uma fibração localmente trivial se para todo $x_{0} \in B$ existe uma vizinhança $U$ de $x_{0}$ tal que $p^{-1}(U)$ é homeomorfa à $U \times p^{-1}\left(x_{0}\right)$ de maneira compatível com as projeções de $U$; além disso a fibra de um ponto depende, em geral, da componente conexa desse ponto, podendo tal fibra ser vazia, ou seja, $p$ não é necessariamente sobrejetora.

Desta forma, considerando os espaços de funções de classe $C^{\infty}$, cuja fonte é uma variedade $V$ com "corners" e cuja meta é uma variedade $W$ (sem bordo), supondo que a fonte $V$ é compacta e munindo os espaços de funções com a topologia $C^{\infty}$ de Whitney, denotaremos por $\operatorname{Emb}(V, W)$ o espaço dos mergulhos de classe $C^{\infty}$ da variedade $V$ na variedade $W$.

Nas condições acima e supondo $M$ uma subvariedade fechada de $V$, o Teorema da Fibração de Cerf afirma que a aplicação canônica $\operatorname{Emb}(V, W) \rightarrow \operatorname{Emb}(M, W)$ é uma fibração localmente trivial.

\subsubsection{O grau de Brouwer}

A referência para esta subseção é [40].

Considere $M$ e $N$ variedades suaves, orientadas, sem bordo e $n$-dimensionais, $N$ conexa.

Definição 1.2.14. Suponha que $f: M \rightarrow N$ é uma aplicação suave e assuma que $M$ é compacta. Seja y um valor regular de $f$. Se $\operatorname{det}\left(d f_{x}\right)>0\left(\operatorname{resp} \cdot \operatorname{det}\left(d f_{x}\right)<0\right)$, então considere o sinal do determinante de $d f_{x}$ igual a 1 (resp. -1), o qual denotaremos por sign $d f_{x}$. Definimos:

$$
\operatorname{deg}(f, y)=\sum_{x \in f^{-1}(y)} \operatorname{sign} d f_{x}
$$

Note que, sendo $M$ compacta, $f: M \rightarrow N$ é uma aplicação própria entre variedades de mesma dimensão, portanto $f^{-1}(y)$ é um conjunto finito de pontos.

O inteiro $\operatorname{deg}(f, y)$ não depende da escolha do valor regular ${ }^{1} y$ e será chamado o grau de Brouwer de $f$, denotado por $\operatorname{deg} f$. Geometricamente, o grau da aplicação representa quantas vezes $M$ se "envolve" em $N$ mediante a aplicação $f$. O sinal (sign) do grau indica se essa ação de "envolver" é realizada preservando a orientação ou invertendo-a.

Teorema 1.2.15. Se $f$ e g são suavemente homotópicas, então $\operatorname{deg} f=\operatorname{deg} g$.

Teorema 1.2.16. Se $M=\partial W$, onde $W$ é uma variedade suave compacta orientada, $e$ $f: M \rightarrow N$ se estende a uma aplicação suave $F: W \rightarrow N$, então $\operatorname{deg} f=0$.

\footnotetext{
${ }^{1}$ Uma prova desta afirmação pode ser encontrada em [40], página 99.
} 


\subsubsection{Transversalidade}

Apresentamos a seguir um conceito que descreve como espaços podem se intersectar.

Definição 1.2.17. Sejam $f: M \rightarrow N$ aplicação suave e $Z \subset N$ subvariedade. Dizemos que $f$ é transversal à $Z$, e denotamos $f \pitchfork Z$, se $\forall x \in f^{-1}(Z)$ temos

$$
d f_{x}\left(T_{x} M\right)+T_{f(x)} Z=T_{f(x)} N .
$$

Teorema 1.2.18. (Teorema de Transversalidade) Sejam $f: M \rightarrow N$ aplicação suave $e$ $Z \subset N$ subvariedade. Se $f \pitchfork Z$, então $f^{-1}(Z)$ é vazio ou uma subvariedade de $M$. Além disso, se $f^{-1}(Z) \neq \emptyset$, sua codimensão em $M$ é igual à codimensão de $Z$ em $N$.

Sejam $M$ variedade, $N \subset M$ e $Z \subset M$ subvariedades de $M$.

Definição 1.2.19. (1) Dizemos que $N$ é transversal à $Z$ em $x \in N \cap Z$ se $T_{x} N+T_{x} Z=$ $T_{x} M$. Usamos a notação $N \pitchfork_{x} Z$.

(2) Se $N$ é transversal a $Z$ em todo $x \in N \cap Z$, dizemos que $N$ é transversal a $Z$ e denotamos $N \pitchfork Z$.

Observações 1.2.20. (1) $N \pitchfork Z \Leftrightarrow i_{N} \pitchfork Z$, onde $i_{N}: N \rightarrow M$ é a aplicação inclusão.

(2) Se $N \pitchfork Z$, então $N \cap Z$ é subvariedade de $M$ e codim $(N \cap Z)=\operatorname{codim}(N)+\operatorname{codim}(Z)$.

(3) Se $f: M \rightarrow N$ é uma aplicação suave e $Z=\{p\} \subset N$ é um ponto, então $f \pitchfork Z \Leftrightarrow p$ é valor regular de $f$.

\subsection{4 h-cobordismo e aplicações}

Com exceção do Teorema de h-cobordismo que será enunciado apenas para fazer sentido a apresentação de algumas aplicações observadas por Milnor, vamos expor aqui somente os resultados que serão utilizados neste trabalho.

Uma terna $\left(W ; V_{0}, V_{1}\right)$ é uma tríade de variedades suaves se $W$ é uma $n$-variedade suave compacta e seu bordo, $\partial W$, é a união disjunta de duas subvariedades fechadas $V_{0}$ e $V_{1}$.

Dadas duas variedades fechadas $M_{0}$ e $M_{1}$ e uma tríade de variedades suaves $\left(W ; V_{0}, V_{1}\right)$, dizemos que a 5 -upla $\left(W ; V_{0}, V_{1} ; h_{0}, h_{1}\right)$ é um cobordismo de $M_{0}$ a $M_{1}$, onde $h_{i}: V_{i} \rightarrow M_{i}$, $i=0,1$, são difeomorfismos. Se além disso, $M_{0}$ e $M_{1}$ são retratos por deformação de $W$, dizemos que a 5-upla é um $h$-cobordismo e que $M_{0}$ e $M_{1}$ são $h$-cobordantes. 
Teorema 1.2.21. (Teorema do h-Cobordismo - [37, p.107]) Suponha W uma n-variedade compacta e $\left(W ; V_{0}, V_{1}\right)$ uma triade de variedades suaves com as seguintes propriedades:

(1) $W, V_{0}$ e $V_{1}$ são simplesmente conexas;

(2) $H_{*}\left(W, V_{0}\right)=0$;

(3) $\operatorname{dim} W=n \geqslant 6$.

Então $W$ é difeomorfa a $V_{0} \times[0,1]$.

Apesar do resultado a seguir ser uma consequência do teorema acima, ele será enunciado como teorema devido à sua importância.

Teorema 1.2.22. Duas $n$-variedades suaves, fechadas e simplesmente conexas com $n \geqslant 5$ que são h-cobordantes são difeomorfas.

Como aplicações, vejamos os resultados a seguir, os quais podem ser encontrados em [37, pp. 108-109].

Proposição 1.2.23. (Caracterização do $n$-disco suave $D^{n}, n \geqslant 6$ ) Suponha $W^{n}$ uma $n$-variedade suave, compacta, simplesmente conexa, $n \geqslant 6$, com um bordo simplesmente conexo. Então as seguintes quatro afirmações são equivalentes.

(1) $W^{n}$ é difeomorfo a $D^{n}$.

(2) $W^{n}$ é homeomorfo a $D^{n}$.

(3) $W^{n}$ é contrátil.

(4) $W^{n}$ tem a homologia (integral) de um ponto.

Proposição 1.2.24. (Conjectura de Poincaré Generalizada) $S e M^{n}, n \geqslant 5$, é uma variedade suave fechada que tem o tipo de homotopia da $S^{n}$, então $M^{n}$ é homeomorfa à $S^{n}$.

O resultado acima é devido a Stephen Smale que generalizou (ver [45]) para dimensão maior que ou igual a cinco, a Conjectura de Poincaré enunciada em 1904 para dimensão três, a qual foi provada positivamente por Grigori Perelman em 2003.

Observações 1.2.25. (1) Nas condições da Proposição 1.2.24, temos que $M^{n}$ é difeomorfa à $S^{n}$ se

- $n=1$ ou $n=2$, pela classificação de variedades nessas dimensões; 
- $n=3$, pela solução da Conjectura de Poincaré;

- $n=5$ ou $n=6$ (ver [37, p.109]).

(2) Existem variedades suaves de dimensão 7 que são homeomorfas à $S^{7}$ mas não são difeomorfas à $S^{7}$. Tais variedades são chamadas esferas exóticas.

Os resultados a seguir podem ser encontrados em [29].

Lema 1.2.26. Se $M$ é uma esfera de homotopia, então $M \sharp(-M)$ borda uma variedade contrátil.

Lema 1.2.27. Uma variedade simplesmente conexa $M$ é h-cobordante à esfera $S^{n}$ se, e somente se, $M$ borda uma variedade contrátil.

\subsection{Fórmulas do tipo Poincaré-Hopf}

Definição 1.3.1. Seja $z$ um zero isolado de um campo de vetores $V$ em uma variedade $M$ de dimensão $n$. $V$ pode ser visto, em coordenadas locais, como uma aplicação de um pequeno conjunto aberto $U \subset \mathbb{R}^{n}$ para um conjunto aberto $U^{\prime} \subset \mathbb{R}^{n}$ onde $0 \in U$ e $0 \in U^{\prime}$ e tal que 0 é o único zero de $V$ em $U$. Definimos o índice de Poincaré-Hopf de $V$ em $z$, e denotamos, por:

$$
\operatorname{deg}_{z}(V)=\operatorname{deg} \frac{V}{|V|}: S_{\varepsilon}^{n-1} \rightarrow S^{n-1}
$$

onde $S_{\varepsilon}^{n-1}$ é uma pequena esfera contida em $U$.

Neste caso, $\operatorname{deg}_{z}(V)$ é também conhecido como o grau topológico de $V$.

É possível checar que a Definição 1.3.1 não depende das coordenadas locais (veja, por exemplo, [38]).

Teorema 1.3.2. (Teorema de Poincaré-Hopf) Seja $M$ uma variedade suave compacta, com $\partial M=\emptyset$. Seja $v$ um campo de vetores tangente e suave em $M$ com um número finito de zeros $z_{1}, \ldots, z_{k}$. Então temos:

$$
\chi(M)=\sum_{i=1}^{k} \operatorname{deg}_{z_{i}}(v) .
$$

Vale ressaltar que a Característica de Euler pode ser vista como uma obstrução para construir um campo de vetores suave tangente à variedade e sem singularidades. Ou seja: se $\chi(M) \neq 0$, todo campo de vetores contínuo e tangente à variedade $M$ admite pelo menos um ponto singular. 
Para $g:\left(\mathbb{R}^{n}, 0\right) \rightarrow(\mathbb{R}, 0)$, um germe de função analítica real com singularidade isolada, a característica de Euler da fibra aparece como um dos invariantes fundamentais, como estabelece a famosa fórmula de Khimshiashvili [30] que relaciona este invariante com o índice de Poincaré-Hopf na origem do campo gradiente da função:

$$
\chi\left(g^{-1}(\delta) \cap B_{\varepsilon}\right)=1-\operatorname{sign}(-\delta)^{n} \operatorname{deg}_{0}(\nabla g),
$$

onde $0<|\delta| \ll \varepsilon \ll 1, \nabla g$ é o gradiente de $g$ e $\operatorname{deg}_{0}(\nabla g)$ é o grau topológico de $\nabla g$.

De forma mais geral, para $f=\left(f_{1}, \ldots, f_{p}\right):\left(\mathbb{R}^{n}, 0\right) \rightarrow\left(\mathbb{R}^{p}, 0\right), \quad 2 \leqslant p<n$, um germe de aplicação analítica real com singularidade isolada na origem, denotando a fibra de Milnor por $M_{f}$ (ver detalhes na Seção 2.1), os autores, em [4], usaram técnicas da teoria de Morse para variedades com bordo para encontrar a seguinte generalização da fórmula de Khimshiashvili, dada em (1.3.1). Tal generalização mostra a relação entre a característica de Euler de uma fibra genérica e o grau topológico das funções coordenadas.

Proposição 1.3.3. Seja $f:\left(\mathbb{R}^{n}, 0\right) \rightarrow\left(\mathbb{R}^{p}, 0\right), n>p \geqslant 2$, um germe de aplicação polinomial com uma singularidade isolada na origem, e considere

$$
f(x)=\left(f_{1}(x), f_{2}(x), \ldots, f_{p}(x)\right)
$$

um representante arbitrário do germe. Denote por $\operatorname{deg}_{0}\left(\nabla f_{i}(x)\right), i=1,2, \ldots, p$, o grau topológico da aplicação $\varepsilon \frac{\nabla f_{i}}{\left\|\nabla f_{i}\right\|}: S_{\varepsilon}^{n-1} \rightarrow S_{\varepsilon}^{n-1}$, para $\varepsilon>0$ suficientemente pequeno.

(i) Se n é par, então $\chi\left(M_{f}\right)=1-\operatorname{deg}_{0} \nabla f_{1}$. Além disso, temos

$$
\operatorname{deg}_{0} \nabla f_{1}=\operatorname{deg}_{0} \nabla f_{2}=\cdots=\operatorname{deg}_{0} \nabla f_{p}
$$

(ii) Se n é ímpar, então $\chi\left(M_{f}\right)=1$. Além disso, temos $\operatorname{deg}_{0} \nabla f_{i}=0, i=1,2, \ldots, p$.

Observamos que a Proposição 1.3.3 generaliza de forma natural a fórmula de Khimshiashvili quando $p=1$ e a fórmula (2.2.4) (página 34) provada por Milnor.

\subsection{Grupos de Lie}

Nesta seção faremos um breve estudo dos grupos de Lie: definições, exemplos, algumas propriedades e resultados básicos, os quais serão apresentados sem demonstração. Para maiores detalhes confira [32, Capítulo 7].

Um grupo de Lie é uma variedade suave $G$ (sem bordo) que é também um grupo no sentido algébrico, com a propriedade que a aplicação multiplicação $m: G \times G \rightarrow G$ dada 
por $m(g, h)=g h$ e a aplicação inversão $i: G \rightarrow G$ dada por $i(g)=g^{-1}$ são suaves. Em particular, um grupo de Lie é um grupo topológico (um espaço topológico com uma estrutura de grupo tal que as aplicações multiplicação e inversão são contínuas).

O elemento identidade de um grupo de Lie arbitrário será denotado por $e$, exceto em alguns exemplos, como o grupo de matrizes (neste caso o elemento identidade é denotado por $I_{n}$ ) e o $\mathbb{R}^{n}$ (denotamos o elemento identidade por 0 ).

Se $G$ é um grupo de Lie, qualquer elemento $g \in G$ define aplicações

$$
\begin{aligned}
L_{g}: G & \rightarrow G & R_{g}: G & \rightarrow G \\
h & \mapsto g h & & h
\end{aligned},,
$$

chamadas translação à esquerda e translação à direita, respectivamente. Essas aplicações, na verdade, são difeomorfismos de $G$.

Vejamos a seguir alguns exemplos de grupos de Lie.

1. O grupo linear geral $G L(n, \mathbb{R})$ é o conjunto de $n \times n$ matrizes invertíveis com entradas reais. Ele é um grupo sob multiplicação de matrizes, e é uma subvariedade aberta do espaço vetorial $M(n, \mathbb{R})$ (espaço das matrizes $n \times n$ com entradas reais). A multiplicação é suave porque as entradas de uma matriz produto $A B$ são polinômios nas entradas de $A$ e $B$. A inversão é suave pela regra de Cramer.

2. Considere $G L^{+}(n, \mathbb{R})$ o subconjunto de $G L(n, \mathbb{R})$ consistindo de matrizes com determinante positivo. Como $\operatorname{det}(A B)=(\operatorname{det} A)(\operatorname{det} B)$ e $\operatorname{det}\left(A^{-1}\right)=1 / \operatorname{det} A$, ele é um subgrupo de $G L(n, \mathbb{R})$; e como ele é a pré-imagem de $(0, \infty)$ pela função determinante contínua, ele é um subconjunto aberto de $G L(n, \mathbb{R})$ e portanto uma variedade $n^{2}$-dimensional. As operações de grupo são as restrições daquelas de $G L(n, \mathbb{R})$, então elas são suaves. Assim, $G L^{+}(n, \mathbb{R})$ é um grupo de Lie.

3. Suponha $\mathrm{G}$ um grupo de Lie arbitrário e $H \subseteq G$ um subgrupo aberto (um subgrupo que é também um conjunto aberto). Pelo mesmo argumento do item 2., $H$ é um grupo de Lie com a estrutura de grupo e a estrutura de variedade suave herdadas de $G$.

4. O espaço Euclidiano $\mathbb{R}^{n}$ com a adição vetorial ordinária como a operação de grupo é um grupo de Lie abeliano não-compacto de dimensão $n$.

Se $G$ e $H$ são grupos de Lie, um homomorfismo de grupo de Lie de $G$ em $H$ é uma aplicação suave $F: G \rightarrow H$ que é também um homomorfismo de grupo. Se ele é também um difeomorfismo (isto é, se ele tem uma inversa que também é um homomorfismo de grupo de Lie), ele é chamado um isomorfismo de grupo de Lie e $G$ e $H$ são ditos grupos de Lie isomorfos. 
Teorema 1.4.1. [32, Teorema 7.5] Todo homomorfismo de grupo de Lie tem posto constante.

Um subgrupo de Lie de um grupo de Lie $G$ é um subgrupo de $G$ munido com uma topologia e estrutura suave que o torna um grupo de Lie e uma subvariedade imersa de G.

Proposição 1.4.2. [32, Proposição 7.11] Seja $G$ um grupo de Lie, e suponha que $H \subseteq G$ é um subgrupo de Lie. Então $H$ é fechado em $G$ se, e só se, ele é mergulhado.

Exemplo 1.4.3 (Subgrupos de Lie).

1. (O grupo ortogonal). Dizemos que uma matriz $A$ real $n \times n$ é ortogonal quando, considerada como uma aplicação linear $A: \mathbb{R}^{n} \rightarrow \mathbb{R}^{n}$, ela preserva o produto escalar Euclidiano: $(A x) \cdot(A y)=x \cdot y$ para todo $x, y \in \mathbb{R}^{n}$. Dizer que $A$ é ortogonal equivale a afirmar que $A^{T} A=I_{n}$, onde $A^{T}$ representa a transposta de $A$.

O grupo ortogonal de grau $n$ é o conjunto $O(n)$ consistindo de todas as matrizes ortogonais $n \times n$ e é um subgrupo de Lie de $G L(n, \mathbb{R})$ de dimensão $\frac{n}{2}(n-1)$. Ele é compacto porque é fechado e limitado em $M(n, \mathbb{R}) \cong \mathbb{R}^{n^{2}}$. Além disso, é um grupo desconexo, pois possui duas componentes conexas:

$$
O^{+}\left(\mathbb{R}^{n}\right)=\{X \in O(n) ; \operatorname{det} X>0\} \text { e } O^{-}\left(\mathbb{R}^{n}\right)=\{X \in O(n) ; \operatorname{det} X<0\} .
$$

A componente conexa $O^{+}\left(\mathbb{R}^{n}\right)$ é, na verdade, um subgrupo de mesma dimensão que $O(n)$, consistindo de matrizes ortogonais de determinantes iguais a 1. Ele é chamado grupo ortogonal especial e denotado por $S O(n)$ (para $n=3$, é o grupo de rotação $S O(3))$.

2. O conjunto $S L(n, \mathbb{R})$ consistindo de matrizes de determinante 1 é um subgrupo de Lie fechado e conexo do grupo $G L(n, \mathbb{R})$, tem dimensão $n^{2}-1$ e é chamado grupo linear especial.

Agora veremos ações de grupo de Lie sobre outras variedades.

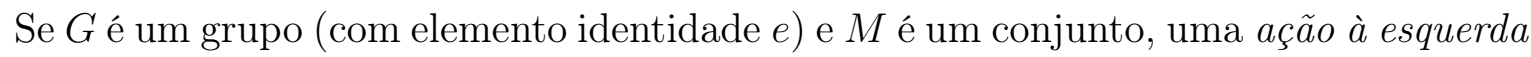
de $G$ em $M$ é uma aplicação $G \times M \rightarrow M$ definida por $(g, p) \mapsto g \cdot p$, que satisfaz

$$
\begin{aligned}
g_{1} \cdot\left(g_{2} \cdot p\right) & =\left(g_{1} g_{2}\right) \cdot p & & \text { para todos } g_{1}, g_{2} \in G \text { e } p \in M ; \\
e \cdot p & =p & & \text { para todo } p \in M .
\end{aligned}
$$

Uma ação à direita de $G$ em $M$ é uma aplicação $M \times G \rightarrow M$ definida por $(p, g) \mapsto p \cdot g$, satisfazendo 


$$
\begin{aligned}
\left(p \cdot g_{1}\right) \cdot g_{2} & =p \cdot\left(g_{1} g_{2}\right) & & \text { para todos } g_{1}, g_{2} \in G \text { e } p \in M \\
p \cdot e & =p & & \text { para todo } p \in M
\end{aligned}
$$

Se $M$ é um espaço topológico e $G$ é um grupo topológico, dizemos que uma ação de $G$ em $M$ é uma ação contínua se as aplicações descritas acima são contínuas. Neste caso, $M$ é chamado um $G$-espaço (à direita ou à esquerda). Se, além disso, $M$ é uma variedade suave (com ou sem bordo), $G$ é um grupo de Lie e as aplicações acima são suaves, a ação é chamada ação suave.

Vejamos alguns exemplos de ações de grupo de Lie.

1. Se $G$ é qualquer grupo de Lie e $M$ é qualquer variedade suave, a ação trivial de $G$ em $M$ é definida por $g \cdot p=p$ para todo $g \in G$ e $p \in M$.

2. Todo grupo de Lie age suavemente sobre ele mesmo tanto pela translação à direita (similarmente à esquerda), quanto pela conjugação: $g \cdot h=g h g^{-1}$.

3. A ação natural de $G L(n, \mathbb{R})$ sobre $\mathbb{R}^{n}$ é a ação à esquerda dada pela multiplicação de matrizes: $(A, x) \mapsto A x$, considerando $x \in \mathbb{R}^{n}$ como uma matriz coluna.

Suponha $G$ um grupo de Lie e $M$ e $N$ variedades suaves munidas com $G$-ações suaves à direita e à esquerda. Uma aplicação $F: M \rightarrow N$ é dita equivariante com respeito às $G$-ações dadas se para cada $g \in G$,

$$
\begin{array}{ll}
F(g \cdot p)=g \cdot F(p) & \text { (para ações à esquerda) } \\
F(p \cdot g)=F(p) \cdot g & \text { (para ações à direita) }
\end{array}
$$

A proposição a seguir é bastante útil e será utilizada adiante para a obtenção de novos resultados. A prova pode ser encontrada em [9, p.225].

Proposição 1.4.4. [9, Proposição 7.5] Seja G um grupo de Lie compacto e conexo. Então $\pi_{2}(G)=0$. 


\section{Fibrados suaves}

Este capítulo está dividido da seguinte forma: na primeira seção, enunciamos os teoremas da existência da fibração de Milnor para germes de aplicações analíticas reais com singularidade não necessariamente isolada, e que concordam com os teoremas apresentados por Milnor em [39]. O teorema que garante a existência da fibração no "tubo de Milnor" utiliza as condições $(a)$ e $(b)$ de Milnor, introduzidas por D. Massey em [35]. Um outro teorema apresentado em [2] pelos autores R. Araújo dos Santos, Y. Chen e M. Tibăr, garante a existência da fibração no complementar do link na esfera, com projeção dada por $\frac{f}{\|f\|}$.

A segunda seção apresenta resultados sobre a topologia da fibra e do link, trilhando alguns avanços desde os estudos feitos por Milnor até o momento.

Na seção 2.3, apresentamos os questionamentos feitos por Milnor e as respectivas respostas encontradas na literatura.

Alguns resultados sobre nós, links e NS-pares são apresentados na seção 2.4 .

Por fim, veremos a definição de linking number e de espaço de configuração, bem como uma relação entre eles. Tal resultado será utilizado no Capítulo 4.

\subsection{A fibração de Milnor: abordagem moderna}

Parte dos resultados apresentados nesta seção foram baseados na Seção 2 do pré-print "Fibrations structure and degree formulae for Milnor fibers" ([17]).

Considere $f=\left(f_{1}, \ldots, f_{p}\right):\left(\mathbb{R}^{n}, 0\right) \rightarrow\left(\mathbb{R}^{p}, 0\right)$ uma aplicação analítica com $2 \leqslant p \leqslant$ $n-1, V=f^{-1}(0)$ e $\Sigma_{f}$ o conjunto dos pontos onde os gradientes $\nabla f_{1}, \nabla f_{2}, \ldots, \nabla f_{p}$ são linearmente dependentes, ou seja, o conjunto dos pontos críticos de $f$. Suponha que $f$ não é constante em uma vizinhança da origem. Seja $r$ a função quadrado da distância à origem e denote por $M(f)$ o conjunto dos pontos onde os gradientes $\nabla r, \nabla f_{1}, \ldots, \nabla f_{p}$ são linearmente dependentes, ou seja, o conjunto dos pontos críticos do par $(f, r)$. 
Note que, por definição, $\Sigma_{f} \subseteq M(f)$.

Definição 2.1.1. ([35]) Considerando $f$ e $r$ como acima e denotando a bola fechada em $\mathbb{R}^{n}$ centrada na origem com raio $\varepsilon$ por $B_{\varepsilon}^{n}$, dizemos que:

i) a aplicação $f$ satisfaz a condição $(a)$ de Milnor na origem (ou que $0 \in \mathbb{R}^{p}$ é um valor crítico isolado de $f)$ se $\Sigma_{f} \subset V$ em uma vizinhança da origem. Neste caso, se $\varepsilon>0$ é tal que $B_{\varepsilon}^{n} \cap\left(\overline{\Sigma_{f} \backslash V}\right)=\emptyset$, então dizemos que $\varepsilon$ é um $(a)$ raio de Milnor para $f$ na origem.

ii) a aplicação $f$ satisfaz a condição $(b)$ de Milnor na origem se 0 é isolado em (ou não pertence a) $V \cap(\overline{M(f) \backslash V})$. Neste caso, se $\varepsilon>0$ é tal que $B_{\varepsilon}^{n} \cap V \cap(\overline{M(f) \backslash V}) \subseteq\{0\}$, dizemos que $\varepsilon$ é um $(b)$ raio de Milnor para $f$ na origem.

iii) $\varepsilon>0$ é um raio de Milnor para $f$ na origem se $f$ satisfaz as condições $(a)$ e $(b)$ de Milnor e $\varepsilon$ é simultaneamente um $(a)$ raio de Milnor e um (b) raio de Milnor para $f$ na origem 0 .

Observe que a aplicação $f$ satisfaz a condição $(b)$ de Milnor na origem se, e só se, para cada $\varepsilon>0$ suficientemente pequeno, existe $\delta>0,0<\delta \ll \varepsilon$ tal que a aplicação restrição $f_{\mid}: S_{\varepsilon}^{n-1} \cap f^{-1}\left(\stackrel{\circ}{B}_{\delta}^{p} \backslash\{0\}\right) \rightarrow \stackrel{\circ}{B}_{\delta}^{p} \backslash\{0\}$ é uma submersão suave (e sobrejetora, caso o link de $f^{-1}(0)$ seja não-vazio).

Satisfeitas as condições $(a)$ e $(b)$ de Milnor, podemos concluir que as fibras, de todos os valores regulares próximos da origem, situadas em $B_{\varepsilon}^{n}$ são suaves e transversais à esfera $S_{\varepsilon}^{n-1}$.

Teorema 2.1.2. ([35, Teorema 4.3]) Suponha que $f=\left(f_{1}, \ldots, f_{p}\right):\left(\mathbb{R}^{n}, 0\right) \rightarrow\left(\mathbb{R}^{p}, 0\right)$ satisfaz as condições $(a)$ e $(b)$ de Milnor, e seja $\varepsilon_{0}>0$ um raio de Milnor para $f$ na origem. Então, para cada $\varepsilon$, com $0<\varepsilon \leqslant \varepsilon_{0}$, existe $\delta, 0<\delta \ll \varepsilon$, tal que

$$
f_{\mid}: B_{\varepsilon}^{n} \cap f^{-1}\left(\stackrel{\circ}{B}_{\delta}^{p} \backslash\{0\}\right) \rightarrow \stackrel{\circ}{B}_{\delta}^{p} \backslash\{0\}
$$

é uma fibração suave localmente trivial.

Ideia da prova. Sendo $\varepsilon_{0}$ um raio de Milnor para $f$ na origem, temos, uma vez que a condição $(a)$ de Milnor é satisfeita, que $\Sigma_{f} \cap B_{\varepsilon_{0}}^{n} \subset V \cap B_{\varepsilon_{0}}^{n}$. Mas, isso significa que a aplicação

$$
f_{\mid}: \stackrel{\circ}{B}_{\varepsilon}^{n} \cap f^{-1}\left(\stackrel{\circ}{B}_{\delta}^{p} \backslash\{0\}\right) \rightarrow \stackrel{\circ}{B}_{\delta}^{p} \backslash\{0\}
$$

é uma submersão suave sobrejetora na bola aberta $\stackrel{\circ}{B}_{\varepsilon}^{n}$. 
Como foi discutido acima, a condição (b) de Milnor satisfeita nos diz que para cada $0<\varepsilon \leqslant \varepsilon_{0}$, existe $\delta, 0<\delta \ll \varepsilon$, tal que

$$
f_{\mid}: S_{\varepsilon}^{n-1} \cap f^{-1}\left(\stackrel{\circ}{B}_{\delta}^{p} \backslash\{0\}\right) \rightarrow \stackrel{\circ}{B}_{\delta}^{p} \backslash\{0\}
$$

é uma submersão suave em $S_{\varepsilon}^{n-1}$ (bordo da bola fechada $B_{\varepsilon}^{n}$ ).

Desta forma, para cada $\varepsilon$, podemos escolher $\delta$ tal que

$$
f_{\mid}: B_{\varepsilon}^{n} \cap f^{-1}\left(\stackrel{\circ}{B}_{\delta}^{p} \backslash\{0\}\right) \rightarrow \stackrel{\circ}{B}_{\delta}^{p} \backslash\{0\}
$$

é uma submersão suave, sobrejetora e própria, e consequentemente, pelo Teorema de Ehresmann (Teorema 1.2.10), uma fibração suave localmente trivial.

Uma consequência imediata do Teorema 2.1.2 é a existência da chamada "Fibração de Milnor no tubo", como segue. Veja também a Figura 2.1.

Corolário 2.1.3. Suponha que $f=\left(f_{1}, \ldots, f_{p}\right):\left(\mathbb{R}^{n}, 0\right) \rightarrow\left(\mathbb{R}^{p}, 0\right)$ satisfaz as condições (a) e (b) de Milnor, e seja $\varepsilon_{0}>0$ um raio de Milnor para $f$ na origem. Então, para cada $\varepsilon$, com $0<\varepsilon \leqslant \varepsilon_{0}$, existe $\delta, 0<\delta \ll \varepsilon$, tal que

$$
f_{\mid}: B_{\varepsilon}^{n} \cap f^{-1}\left(S_{\delta}^{p-1}\right) \rightarrow S_{\delta}^{p-1}
$$

é uma fibração suave localmente trivial.

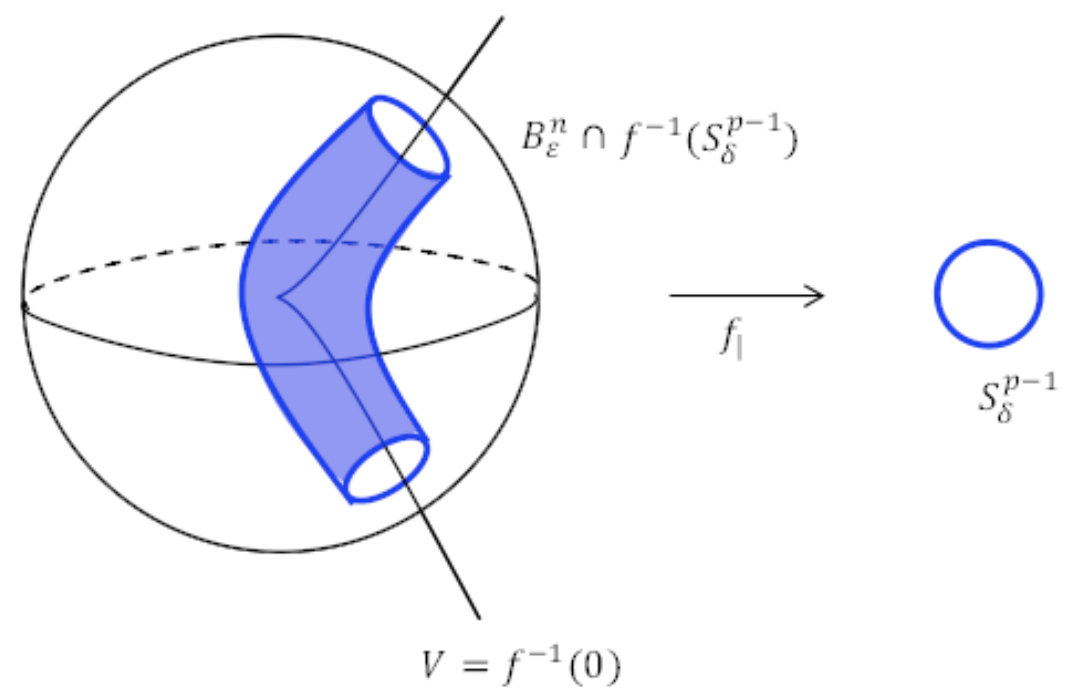

Figura 2.1: Fibração no tubo de Milnor

Agora, seja $\psi:\left(\mathbb{C}^{n+1}, 0\right) \rightarrow(\mathbb{C}, 0)$ um germe de função holomorfa. Podemos considerar $\psi$ como um germe de aplicação de $\mathbb{R}^{2 n+2}$ em $\mathbb{R}^{2}$. É natural fazer a seguinte pergunta: 


\section{$\psi$ satisfaz as condições $(a)$ e (b) de Milnor na origem?}

Antes de apresentarmos a resposta, vejamos a seguinte definição.

Definição 2.1.4. (Desigualdade de Łojasiewicz) Seja $f: U \rightarrow \mathbb{C}$ função holomorfa, com $0 \in U \subset \mathbb{C}^{n+1}, f(0)=0$. Dizemos que $f$ satisfaz a desigualdade de Łojasiewicz na origem se existem uma vizinhança $W$ de 0 contida em $U, 0<\theta<1$ e $c>0$, tal que para todo $z \in W$ temos:

$$
\|\nabla f(z)\| \geqslant c|f(z)|^{\theta}
$$

A resposta da questão acima é sim, pois as condições $(a)$ e (b) de Milnor podem ser vistas como uma aplicação da desigualdade de Łojasiewicz, a qual é satisfeita por toda função holomorfa definida numa vizinhança da origem (ver, por exemplo, [33]). Da desigualdade, segue a condição $(a)$ de Milnor. Como a desigualdade de Łojasiewicz implica a condição $a_{f}$ de Thom para uma $(a)$ estratificação de Whitney de $V$ (ver detalhes em [22, p. 323]), segue a condição (b) de Milnor.

A existência da fibração (na esfera) no caso complexo foi mostrada por Milnor em [39, $\S 4]$. Mais precisamente, dado um representante de um germe de função holomorfa $\psi$ : $U \subset \mathbb{C}^{n+1} \rightarrow \mathbb{C}$ com $\psi(0)=0$, Milnor mostrou que existe um número real suficientemente pequeno $\varepsilon_{0}>0$ tal que para todo $0<\varepsilon \leqslant \varepsilon_{0}$,

$$
\frac{\psi}{\|\psi\|}: S_{\varepsilon}^{2 n+1} \backslash\{\psi=0\} \rightarrow S^{1}
$$

é uma projeção suave de um fibrado localmente trivial.

Alguns anos mais tarde, Lê D. Tráng mostrou que a fibração

$$
\psi_{\mid}: \stackrel{\circ}{B}_{\varepsilon}^{2 n+2} \cap \psi^{-1}\left(S_{\delta}^{1}\right) \rightarrow S_{\delta}^{1}
$$

é fibra equivalente à projeção dada em (2.1.3). Para mais detalhes, o leitor pode consultar [13, Capítulo 3, §1].

Proposição 2.1.5. Seja $f:\left(\mathbb{R}^{n}, 0\right) \rightarrow\left(\mathbb{R}^{p}, 0\right)$ um germe de aplicação analítica com uma singularidade isolada na origem, representado por $f=\left(f_{1}, \ldots, f_{p}\right)$. Então $f$ satisfaz as condições $(a)$ e (b) de Milnor.

Demonstração. A condição (a) de Milnor é trivialmente satisfeita. Como $V=f^{-1}(0)$ é transversal a todas as pequenas esferas, segue a condição (b) de Milnor.

Uma prova do resultado a seguir é de fácil verificação e pode ser feita como uma aplicação direta das técnicas do Milnor. 
Lema 2.1.6. Considere $f$ como na Proposição 2.1.5. Para qualquer $\varepsilon>0$ suficientemente pequeno e qualquer $\rho$ com $0<\rho<\varepsilon$, existe $\zeta>0$ tal que a composição

$$
\left(B_{\varepsilon}^{n} \backslash \operatorname{Int} B_{\rho}^{n}\right) \cap f^{-1}\left(B_{\zeta}^{p}\right) \stackrel{h}{\rightarrow}(K \times I) \times B_{\zeta}^{p} \stackrel{\pi}{\rightarrow} B_{\zeta}^{p}
$$

é a restrição de $f$, onde $h$ é um homeomorfismo, $K=S_{\varepsilon}^{n-1} \cap f^{-1}(0), I=[0,1]$ e $\pi$ é a projeção.

Um teorema da fibração (no tubo) no caso real, foi provado por Milnor em [39, §11]. Ele considerou uma aplicação polinomial real $f: \mathbb{R}^{n} \rightarrow \mathbb{R}^{p}$ tal que $f(0)=0$, com $n \geqslant p \geqslant 2$, e assumiu que numa pequena vizinhança $U$ de $0 \in \mathbb{R}^{n}$, o conjunto singular consistia somente da origem.

Mais precisamente, a existência da fibração de Milnor no caso real foi apresentada por Milnor da seguinte forma:

Teorema 2.1.7. ([39, Teorema 11.2]) O complemento de uma vizinhança tubular aberta do link $K_{\varepsilon}=f^{-1}(0) \cap S_{\varepsilon}^{n-1}$ em $S_{\varepsilon}^{n-1}$ é o espaço total de um fibrado suave sobre a esfera $S^{p-1}$, cada fibra $F_{f}$ sendo uma variedade suave, compacta, $(n-p)$-dimensional, com $\partial F_{f} \approx K_{\varepsilon}$.

Quando Milnor demonstrou o teorema acima, inicialmente ele provou a existência da fibração no "tubo de Milnor", depois construiu um campo de vetores conveniente em $B_{\varepsilon}^{n} \backslash V$ e usou seu fluxo para "inflar" o tubo para a esfera $S_{\varepsilon}^{n-1}$, mantendo o bordo do tubo fixado. A existência da fibração no complementar do link na esfera $S_{\varepsilon}^{n-1}$ não foi detalhada por Milnor, mas ele advertiu o leitor que com o processo de inflar o tubo, não seria possível garantir que a projeção do fibrado suave localmente trivial fosse dada por $\frac{f}{\|f\|}$.

No caso mais geral de singularidade não-isolada, os autores Araújo dos Santos e M. Tibăr, em [6], forneceram uma caracterização de uma fibração com projeção dada por $\frac{f}{\|f\|}$. Com as mesmas notações utilizadas no início desta seção e sendo $K_{\varepsilon}=f^{-1}(0) \cap S_{\varepsilon}^{n-1}$, eles provaram que para todo $\varepsilon>0$ suficientemente pequeno e assumindo que $\Sigma_{f} \cap V \subseteq\{0\}$,

$$
\frac{f}{\|f\|}: S_{\varepsilon}^{n-1} \backslash K_{\varepsilon} \rightarrow S^{p-1}
$$

é uma fibração suave localmente trivial se, e só se, é uma submersão suave.

A fim de enunciarmos um teorema sobre a existência da fibração na esfera para singularidade não-isolada, provado por Araújo dos Santos, Chen e Tibăr em [2], vamos apresentar uma definição considerada por eles no mesmo artigo.

Definição 2.1.8. ([2]) Dizemos que o par $(K, \theta)$ é uma estrutura open book superior com "binding" singular em uma variedade analítica $M$ de dimensão $m-1 \geqslant p \geqslant 2$ se $K \subset M$ 
é uma subvariedade real singular de codimensão $p$ e $\theta: M \backslash K \rightarrow S_{1}^{p-1}$ é uma fibração suave localmente trivial tal que $K$ admite uma vizinhança $N$ de modo que a restrição $\theta_{\left.\right|_{N \backslash K}}$ é a composição $N \backslash K \stackrel{h}{\rightarrow} B^{p} \backslash\{0\} \rightarrow S_{1}^{p-1}$, onde $h$ é uma fibração localmente trivial. A subvariedade singular $K$ é chamada "binding" e as (os fechos das) fibras de $\theta$ são chamadas páginas da open book.

Esta definição estende a definição de NS-par que será apresentada na Seção 2.4.

O conjunto dos pontos críticos do $\operatorname{par}\left(\frac{f}{\|f\|}, r\right)$, onde $\left(\frac{f}{\|f\|}, r\right): U \backslash V \rightarrow S^{p-1} \times \mathbb{R}$ e $r(x)=x_{1}^{2}+\cdots+x_{n}^{2}$ é a função quadrado da distância à origem, será denotado por $M\left(\frac{f}{\|f\|}\right)$.

Teorema 2.1.9. ([2, Teorema 1.3]) Seja $f:\left(\mathbb{R}^{n}, 0\right) \rightarrow\left(\mathbb{R}^{p}, 0\right)$ um germe de aplicação analitica real, $n>p \geqslant 2$, tal que $\operatorname{codim} V=p$. Suponha que

$$
(\overline{M(f) \backslash V}) \cap V=\{0\}
$$

Se o germe de $M\left(\frac{f}{\|f\|}\right)$ na origem é vazio para todo $\varepsilon>0$ suficientemente pequeno, então $\left(K_{\varepsilon}, \frac{f}{\|f\|}\right)$ é uma estrutura open book superior nas esferas $S_{\varepsilon}^{n-1}$ com "binding" singular. Em particular,

$$
\frac{f}{\|f\|}: S_{\varepsilon}^{n-1} \backslash K_{\varepsilon} \rightarrow S^{p-1}
$$

é a projeção de uma fibração suave localmente trivial.

Demonstração. Podemos assumir que $K_{\varepsilon}$ é não-vazio. De fato, no caso em que ele é vazio, a condição (2.1.4) é sempre satisfeita e pela hipótese $M\left(\frac{f}{\|f\|}\right)=\emptyset$, temos que $\frac{f}{\|f\|}: S_{\varepsilon}^{n-1} \rightarrow S^{p-1}$ é uma submersão própria e sobrejetora, pois $S^{p-1}$ é conexa. Logo, o resultado segue do Teorema de Ehresmann (Teorema 1.2.10).

No caso em que $K_{\varepsilon}$ é não-vazio, a projeção $\frac{f}{\|f\|}: S_{\varepsilon}^{n-1} \backslash K_{\varepsilon} \rightarrow S^{p-1}$ não é própria e desta forma, não podemos aplicar o Teorema 1.2.10 diretamente. Porém, a condição (2.1.4) é suficiente para implicar que a restrição

$$
f_{\mid}: S_{\varepsilon}^{n-1} \cap f^{-1}\left(\stackrel{\circ}{B}_{\eta}^{p} \backslash\{0\}\right) \rightarrow \stackrel{\circ}{B}_{\eta}^{p} \backslash\{0\}
$$

é, para todo $0<\eta \ll \varepsilon$, uma submersão suave, própria e sobrejetora e, portanto, uma fibração suave localmente trivial pelo Teorema 1.2.10. Assim, para todo $0<\delta<\eta$, temos que a aplicação

$$
f_{\mid}: S_{\varepsilon}^{n-1} \cap f^{-1}\left(B_{\delta}^{p} \backslash\{0\}\right) \rightarrow B_{\delta}^{p} \backslash\{0\}
$$

também é uma fibração suave localmente trivial. 
Compondo a fibração (2.1.6) com a projeção radial $\pi_{1}: B_{\delta}^{p} \backslash\{0\} \rightarrow S^{p-1}, \pi_{1}(y)=\frac{y}{\|y\|}$, obtemos que

$$
\frac{f}{\|f\|}: S_{\varepsilon}^{n-1} \cap f^{-1}\left(B_{\delta}^{p} \backslash\{0\}\right) \rightarrow S^{p-1}
$$

é uma fibração localmente trivial.

Utilizando a fibração $f_{\mid}: S_{\varepsilon}^{n-1} \cap f^{-1}\left(S_{\delta}^{p-1}\right) \rightarrow S_{\delta}^{p-1}$ e escalonando a meta para a esfera unitária, obtemos que a aplicação

$$
\frac{f}{\|f\|}: S_{\varepsilon}^{n-1} \cap f^{-1}\left(S_{\delta}^{p-1}\right) \rightarrow S^{p-1}
$$

é também uma fibração localmente trivial.

Por outro lado, a hipótese que $M\left(\frac{f}{\|f\|}\right)=\emptyset$ significa que a aplicação

$$
\frac{f}{\|f\|}: S_{\varepsilon}^{n-1} \backslash f^{-1}\left(\stackrel{\circ}{B_{\delta}^{p}}\right) \rightarrow S^{p-1}
$$

é uma submersão suave própria, e consequentemente sobrejetora, por (2.1.8).

Colando as aplicações (2.1.7) e (2.1.9) ao longo do bordo comum $S_{\varepsilon}^{n-1} \cap f^{-1}\left(S_{\delta}^{p-1}\right)$, usando a aplicação (2.1.8), obtemos o resultado desejado (ver Figura 2.2).

Observação 2.1.10. Em [2], foi provado o análogo do Teorema da fibração de Milnor na esfera quando $\operatorname{dim} \Sigma_{f} \geqslant 0$.

Além disso, um germe de função holomorfa $\psi:\left(\mathbb{C}^{n+1}, 0\right) \rightarrow(\mathbb{C}, 0)$ satisfaz diretamente as hipóteses do Teorema 2.1.9. A hipótese (2.1.4) é satisfeita porque ela é a condição (b) de Milnor e esta discussão já foi feita nesta seção. A segunda hipótese é satisfeita porque a projeção $\frac{f}{\|f\|}$ é submersão para todo $\varepsilon>0$ suficientemente pequeno, conforme [39, Lema $4.3]$. 

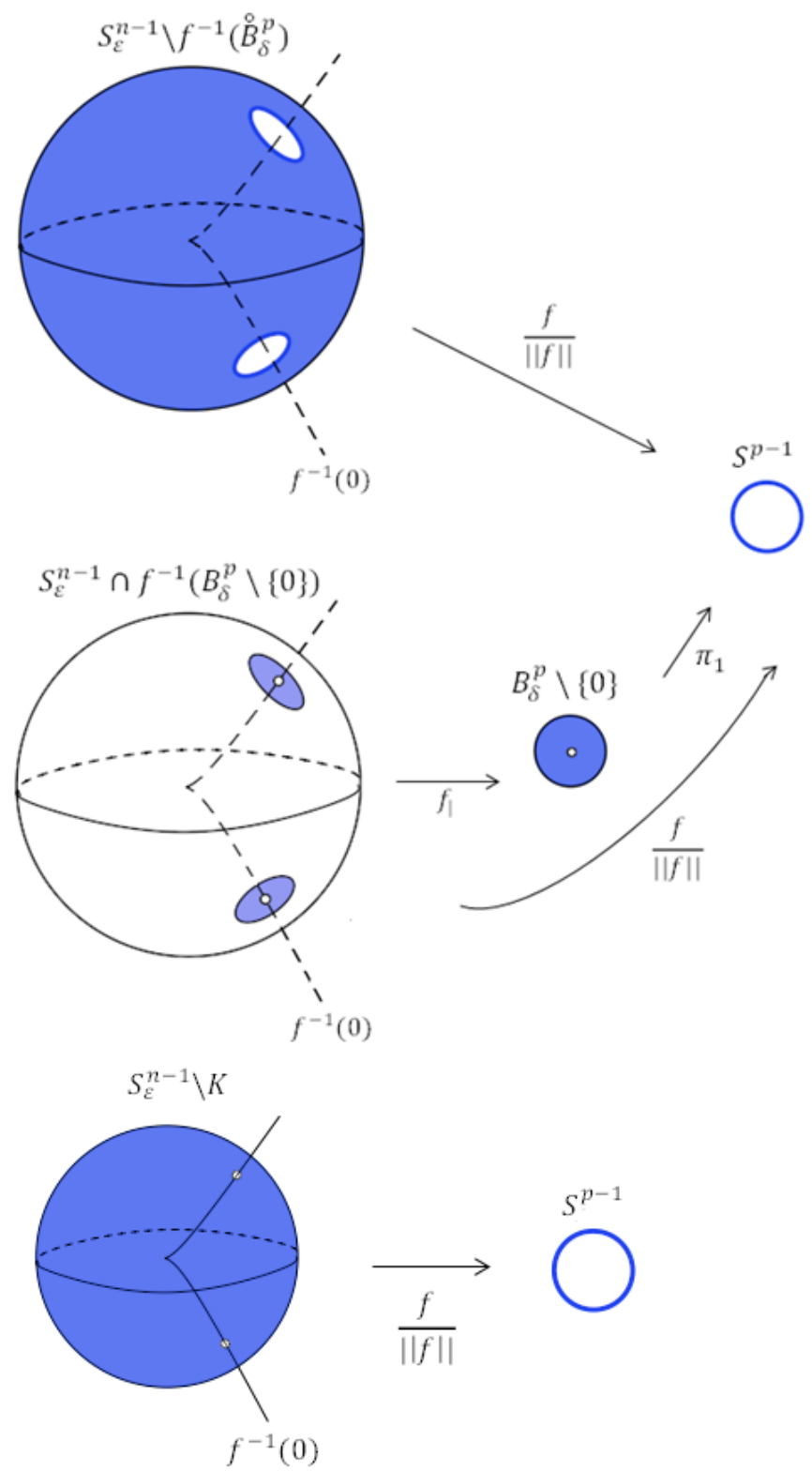

Figura 2.2: As fibrações (2.1.7) e (2.1.9) e a fibração de Milnor no complementar do link na esfera 


\subsection{A topologia da fibra e do link}

Nesta seção, no caso complexo, denotaremos o link $K_{\varepsilon}$ por $K$, pois é bem conhecido que seu tipo topológico não muda, $\forall \varepsilon>0$ suficientemente pequeno.

Ainda no caso complexo, continuando com o estudo da fibração suave localmente trivial $\frac{\psi}{\|\psi\|}: S_{\varepsilon}^{2 n+1} \backslash K \rightarrow S^{1}$ e usando ferramentas da teoria de Morse, Milnor provou em [39] que $F_{\theta}=\left(\frac{\psi}{\|\psi\|}\right)^{-1}\left(e^{i \theta}\right)$ é uma variedade suave paralelizável de dimensão real $2 n$ e que tem o tipo de homotopia de um $C W$-complexo finito de dimensão (no máximo) $n$. Além disso, o espaço topológico $K$ é $(n-2)$-conexo.

Milnor também mostrou em $[39, \S 5]$ que se o número complexo $c \neq 0$ está suficientemente próximo de zero, então a hipersuperfície complexa $\psi^{-1}(c)$ intersecta o $\varepsilon$-disco aberto em uma variedade suave que é difeomorfa à fibra $F_{\theta}$ (ver Figura 2.3).

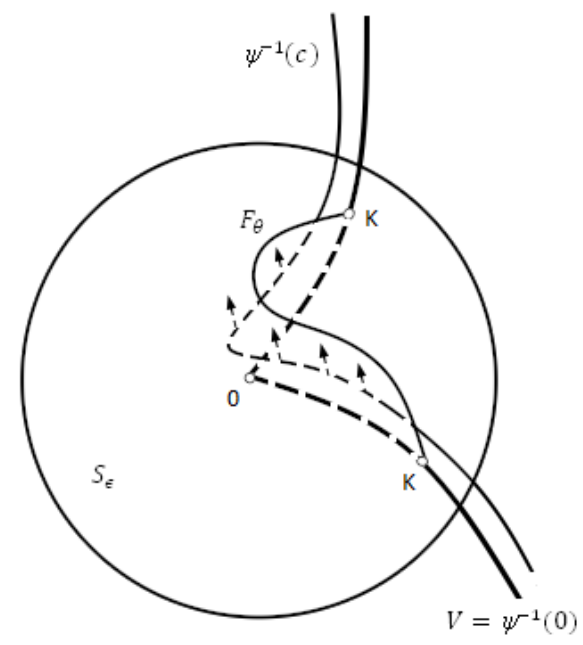

Figura 2.3: Figura do livro do Milnor, página 54

Agora, especializando para uma função holomorfa $\psi: U \subset \mathbb{C}^{n+1} \rightarrow \mathbb{C} \operatorname{com} \psi(0)=0$ e com ponto singular isolado na origem, Milnor forneceu mais detalhes sobre a topologia da fibra. Os resultados em $[39, \S 6]$ são mostrados nos dois lemas a seguir.

Lema 2.2.1. Para $\varepsilon$ suficientemente pequeno, o fecho $\bar{F}_{\theta}$ da fibra $F_{\theta}$ em $S_{\varepsilon}^{2 n+1}$ é uma variedade $2 n$-dimensional com bordo, cujo interior é $F_{\theta}$ e cujo bordo é o link $K$.

A linha da demonstração abaixo é análoga à apresentada em [39, pp.55-56].

Demonstração. Note que os pontos críticos de $\psi_{\mid S_{\varepsilon}^{2 n+1}}$ são aqueles pontos $z \in S_{\varepsilon}^{2 n+1}$ para os quais o vetor (não-nulo) $\nabla \psi$ é um múltiplo complexo de $z$. 
Afirmação: $\psi_{\mid S_{\varepsilon}}: S_{\varepsilon}^{2 n+1} \rightarrow \mathbb{C}$ não possui pontos críticos em $K$ para $\varepsilon$ suficientemente pequeno.

Prova. Considere os conjuntos algébricos $V=\psi^{-1}(0), \quad W=\left\{z \in \mathbb{C}^{n+1} ; \nabla \psi(z)=\right.$ $\lambda z, \lambda \in \mathbb{C}\}$ e o conjunto $U=\left\{z \in \mathbb{C}^{n+1} ;\|z\|^{2}>0\right\}=\mathbb{C}^{n+1} \backslash\{0\}$. Note que $U \cap V \cap W$ é um conjunto semi-algébrico. Usaremos o Lema de Seleção da Curva (ver [39, Lema 3.1]): Suponha que $\forall \varepsilon>0$ suficientemente pequeno, existe ponto crítico $z$ de $\psi_{\mid S_{\varepsilon}}$ em $V$. Assim, $0 \in \overline{U \cap V \cap W}$ e portanto, existe um caminho $p:\left[0, \varepsilon^{\prime}\right] \rightarrow \mathbb{C}^{n+1} \operatorname{com} p(0)=0$ e $p(t) \in$ $U \cap V \cap W$ para $t>0$.

Desta forma,

$$
\begin{aligned}
\psi(p(t)) \equiv 0 \Rightarrow 0 & =\frac{d \psi}{d t}(p(t))=\left\langle\frac{d p}{d t}(t), \nabla \psi(p(t))\right\rangle \\
& =\left\langle\frac{d p}{d t}(t), \lambda p(t)\right\rangle=\bar{\lambda}\left\langle\frac{d p}{d t}(t), p(t)\right\rangle
\end{aligned}
$$

Sendo $\lambda \neq 0$ para $t>0$, temos da equação $(2.2 .1)$ que $0=\left\langle\frac{d p}{d t}(t), p(t)\right\rangle$ e consequentemente

$$
0=2 \cdot \operatorname{Re}\left\langle\frac{d p}{d t}(t), p(t)\right\rangle=\frac{d}{d t}\|p(t)\|^{2} .
$$

Logo $p(t)$ é constante, e sendo $p(0)=0$, temos que $p(t) \equiv 0$. Mas isso é uma contradição pois $p(t) \in U$ para $t>0$. Isso conclui a prova da afirmação.

Agora, seja $z^{0} \in K$. Como $z^{0}$ não é ponto crítico de $\psi_{\mid S_{\varepsilon}^{2 n+1}}$, existem, pela forma local das submersões, coordenadas locais (reais) $u_{1}, \ldots, u_{2 n+1}$ para $S_{\varepsilon}^{2 n+1}$ em uma vizinhança $U$ de $z^{0}$ de modo que $\psi(z)=u_{1}(z)+i u_{2}(z)$ para todo $z \in U$.

Vamos considerar a fibra $F_{0}=\left(\frac{\psi}{\|\psi\|}\right)^{-1}(1)=\left\{z \in S_{\varepsilon}^{2 n+1} \backslash K ; \psi(z)=\|\psi(z)\|\right\}$. Note que $z \in F_{0} \cap U \Leftrightarrow u_{1}(z)>0$ e $u_{2}(z)=0$. Por outro lado, $z \in K \cap U \Leftrightarrow u_{1}(z)=0$ e $u_{2}(z)=0$. Assim, $\overline{F_{0}}$ intersecta $U$ no conjunto $u_{1} \geqslant 0, u_{2}=0$.

Logo $\overline{F_{0}} \cap U$ é uma variedade suave $2 n$-dimensional com interior $F_{0} \cap U$ e bordo $K \cap U$. Como todo ponto de $K$ admite uma tal vizinhança $U$, segue o resultado para a fibra $F_{0}$.

Para as outras fibras $F_{\theta}$, faz-se uma discussão similar.

A variedade compacta com bordo, $\bar{F}_{\theta}$, está mergulhada em $S_{\varepsilon}^{2 n+1}$ de tal modo que tem o mesmo tipo de homotopia de seu complemento $S_{\varepsilon}^{2 n+1} \backslash \bar{F}_{\theta}$. De fato, a aplicação $S_{\varepsilon}^{2 n+1} \backslash\left(K \cup F_{\theta}\right) \rightarrow S^{1} \backslash\left\{e^{i \theta}\right\}$ ainda é um fibrado localmente trivial por ser a restrição do fibrado $S_{\varepsilon}^{2 n+1} \backslash K \rightarrow S^{1}$. Como $S^{1} \backslash\left\{e^{i \theta}\right\}$ é contrátil, o primeiro fibrado é trivial e portanto, $S_{\varepsilon}^{2 n+1} \backslash \overline{F_{\theta}}$ é difeomorfo a $\left(S^{1} \backslash\left\{e^{i \theta}\right\}\right) \times F_{\theta^{\prime}}$, sendo $F_{\theta^{\prime}}$ qualquer fibra diferente de $F_{\theta}$. Assim $F_{\theta^{\prime}}$ é retrato por deformação de $S_{\varepsilon} \backslash \overline{F_{\theta}}$ e temos

$$
S_{\varepsilon} \backslash \overline{F_{\theta}} \simeq F_{\theta^{\prime}} \simeq F_{\theta} \simeq \overline{F_{\theta}}
$$


na qual a última relação segue do Lema 2.2.1 e do fato que uma variedade com bordo tem o mesmo tipo de homotopia do seu interior.

Por (2.2.2) e pela dualidade de Alexander, temos os seguintes isomorfismos entre os grupos de homologia e cohomologia reduzidas

$$
\widetilde{H}_{i}\left(F_{\theta}\right) \cong \widetilde{H}_{i}\left(S_{\varepsilon}^{2 n+1} \backslash \overline{F_{\theta}}\right) \cong \widetilde{H}^{2 n-i}\left(\overline{F_{\theta}}\right)
$$

Conforme [39, Teorema 5.1], a fibra $F_{\theta}$ tem o tipo de homotopia de um CW-complexo de dimensão $n$. Desta forma, a relação (2.2.3) é isomorfa a zero para $2 n-i>n$, i.e., para $i<n$. Assim, a fibra $F_{\theta}$ tem a homologia de um ponto em dimensão menor que $n$ e este resultado pode ser melhorado como segue.

Lema 2.2.2. A fibra $F_{\theta}$ é $(n-1)$-conexa.

Ideia da prova. Pela discussão acima é suficiente provar que $F_{\theta}$ é simplesmente conexa para $n \geqslant 2$, pois o resultado seguirá do Teorema de Whitehead (ver Teorema 1.1.9).

Para provar que $F_{\theta}$ é simplesmente conexa, usamos a função de Morse $h_{\theta}: \bar{F}_{\theta} \rightarrow \mathbb{R}_{-}$, em que $h_{\theta}=-s_{\theta}, s_{\theta}(z)=|\psi(z)|$ e $\mathbb{R}_{-}$é o conjunto dos números reais não-positivos, e a técnica da adjunção de "handles" de índice de Morse $\leqslant n$ para obter $\bar{F}_{\theta}$ a partir de um disco $D_{0}^{2 n}$, ganhando que $\pi_{1}\left(S_{\varepsilon}^{2 n+1} \backslash \bar{F}_{\theta}\right)$ é trivial para $n \geqslant 2$. O resultado segue do fato de $S_{\varepsilon}^{2 n+1} \backslash \bar{F}_{\theta}$ e $\bar{F}_{\theta}$ serem conexos por caminho e da relação $(2.2 .2)$.

Alguns anos mais tarde, Kato e Matsumoto estenderam o resultado anterior como segue:

Teorema 2.2.3. ([28, Teorema 1]) Seja $x_{0} \in V=\psi^{-1}(0)$ um ponto singular de uma função holomorfa $\psi: U \subset \mathbb{C}^{n+1} \rightarrow \mathbb{C}$. Suponha que a dimensão complexa do germe do conjunto singular $\Sigma$ em $x_{0}, \quad \operatorname{dim}_{\mathbb{C}, x_{0}} \Sigma$, é igual a s. Então a fibra de Milnor $F$ de $\psi$ em $x_{0}$ é pelo menos $(n-s-1)$-conexa.

Milnor também provou que a fibra $F_{\theta}$ tem o tipo de homotopia de um buquê $S^{n} \vee$ $S^{n} \vee \cdots \vee S^{n}$ de esferas. Assim,

$$
H_{q}\left(F_{\theta} ; \mathbb{Z}\right)=H_{q}\left(S^{n} \vee \cdots \vee S^{n} ; \mathbb{Z}\right) \cong\left\{\begin{array}{l}
\mathbb{Z}, \text { se } q=0, \\
\mathbb{Z}^{\mu}=\mathbb{Z} \oplus \cdots \oplus \mathbb{Z}, \text { se } q=n, \\
0, \text { se } q \neq 0, n,
\end{array}\right.
$$

onde $\mu$ (conhecido como o número de Milnor) é o número de esferas no buquê. Além disso, em [39, §7 e Apêndice B], ele mostrou que esse número apenas é zero se a origem é 
um ponto regular de $\psi$. Mais especificamente $\mu=\operatorname{deg}_{0}(\nabla \psi(z))$, onde o segundo membro da igualdade representa o grau topológico da aplicação

$$
\varepsilon \frac{\nabla \psi}{\|\nabla \psi\|}: S_{\varepsilon}^{2 n+1} \rightarrow S_{\varepsilon}^{2 n+1}
$$

para todo $\varepsilon>0$ suficientemente pequeno, e

$$
\nabla \psi=\left(\frac{\partial \psi}{\partial z_{1}}, \frac{\partial \psi}{\partial z_{2}}, \ldots, \frac{\partial \psi}{\partial z_{n+1}}\right)
$$

Com isso, temos que a característica de Euler da fibra de Milnor é dada por

$$
\chi\left(F_{\theta}\right)=1+(-1)^{n} \operatorname{deg}_{0}(\nabla \psi(z)) .
$$

Vimos na Seção 1.3 que os autores R. Araújo dos Santos, N. Dutertre e D. Dreibelbis forneceram expressões similares à (2.2.4) para a fibra de Milnor real. Além disso, em [17, Seção 2.3], os autores mostram que dada uma função holomorfa com singularidade isolada, a fórmula de Milnor (2.2.4) segue da Proposição 1.3.3, item (i).

Embora no caso complexo a fibração de Milnor e os vários resultados sobre a topologia da fibra de Milnor sejam bem entendidos, como acabamos de ver, o mesmo não pode ser dito no caso real. Por exemplo, no que se refere à existência da fibração de Milnor real para singularidades não isoladas, apenas recentemente foram obtidos alguns resultados.

Para singularidades isoladas, como já mencionamos na Introdução, Milnor mostrou em [39] que para $n<2(p-1)$, a fibra é sempre contrátil. Além disso, decorre da prova de [39, Lema 11.4, p. 100] o seguinte resultado.

Lema 2.2.4. Seja $f:\left(\mathbb{R}^{n}, 0\right) \rightarrow\left(\mathbb{R}^{p}, 0\right), 2 \leqslant p \leqslant n$, um germe de aplicação polinomial com uma singularidade isolada na origem. Se o link $K_{\varepsilon}$ é não-vazio, então a fibra de Milnor associada, $F_{f}$, é $(p-2)$-conexa.

Demonstração. Sabemos que $\operatorname{dim} K_{\varepsilon}=n-p-1$. Considere uma decomposição celular $\cup_{l=0}^{n-p-1} C_{l}$ do link $K_{\varepsilon}$, onde $C_{l}$ é uma união disjunta finita de células de dimensão $l$, e $N\left(K_{\varepsilon}\right)$ sua vizinhança tubular. Como $N\left(K_{\varepsilon}\right)=D_{\delta}^{p} \times K_{\varepsilon}=\cup_{l=0}^{n-p-1}\left(D_{\delta}^{p} \times C_{l}\right)$, segue que a esfera $S_{\varepsilon}^{n-1}$ pode ser obtida do subespaço $S_{\varepsilon}^{n-1} \backslash \operatorname{Int} N\left(K_{\varepsilon}\right)$ através da adjunção de um número finito de células de dimensão $\geqslant p$, uma $(p+l)$-célula para cada $l$-célula de $K_{\varepsilon}$. Portanto essa adjunção não altera os grupos de homotopia em dimensão menor ou igual a $p-2, \log 0$

$$
\pi_{i}\left(S_{\varepsilon}^{n-1} \backslash \operatorname{Int} N\left(K_{\varepsilon}\right)\right) \cong \pi_{i} S_{\varepsilon}^{n-1}=0 \text { para } i \leqslant p-2 .
$$

Como a fibração $S_{\varepsilon}^{n-1} \backslash \operatorname{Int} N\left(K_{\varepsilon}\right) \rightarrow S^{p-1}$ tem uma seção

$$
S^{p-1} \rightarrow \partial\left(S_{\varepsilon}^{n-1} \backslash \operatorname{IntN}\left(K_{\varepsilon}\right)\right) \subset S_{\varepsilon}^{n-1} \backslash \operatorname{Int} N\left(K_{\varepsilon}\right),
$$


temos a sequência exata curta

$$
0 \rightarrow \pi_{i} F_{f} \rightarrow \pi_{i}\left(S_{\varepsilon}^{n-1} \backslash \operatorname{Int} N\left(K_{\varepsilon}\right)\right) \rightarrow \pi_{i} S^{p-1} \rightarrow 0
$$

Sendo $\pi_{i}\left(S_{\varepsilon}^{n-1} \backslash \operatorname{Int} N\left(K_{\varepsilon}\right)\right)=0$ para $i \leqslant p-2$, temos que a fibra $F_{f}$ é também $(p-2)$ conexa.

Observação 2.2.5. É importante notar que o caso do par $(n, p)=(4,3)$ demonstra que a hipótese do link ser não-vazio no Lema 2.2.4 não pode ser removida. De fato, considerando o link vazio, temos que, neste caso, a fibração associada é a fibração de Hopf $S^{1} \hookrightarrow S^{3} \rightarrow$ $S^{2}$, cuja fibra não é simplesmente conexa.

Em [18], os autores Araújo dos Santos, Dutertre, Chen e Andrade provaram o resultado a seguir, que apresenta a relação entre a fibra da fibração no tubo e a fibra da fibração na esfera com projeção $\frac{f}{\|f\|}$ quando as condições $(a)$ e $(b)$ de Milnor são satisfeitas.

Proposição 2.2.6. ([18, Proposição 5.2]) A fibra da fibração (2.1.2) é equivalente homotópica à fibra da fibração (2.1.5).

\subsection{Sobre os questionamentos de Milnor}

Nesta seção, expomos os problemas colocados por Milnor em [39, §11] e apresentamos as respectivas respostas que conseguimos encontrar na literatura.

Seja $f:\left(\mathbb{R}^{n}, 0\right) \rightarrow\left(\mathbb{R}^{p}, 0\right), n>p \geqslant 2$, um germe de aplicação polinomial com uma singularidade isolada na origem e considere $\pi:\left(\mathbb{R}^{p}, 0\right) \rightarrow\left(\mathbb{R}^{p-1}, 0\right), p \geqslant 3$, o germe da projeção canônica. Claramente, o germe da aplicação composição $g=\pi \circ f:\left(\mathbb{R}^{n}, 0\right) \rightarrow$ $\left(\mathbb{R}^{p-1}, 0\right)$ também tem singularidade isolada na origem e assim, temos duas fibrações:

$$
\begin{gathered}
f: f^{-1}\left(S_{\delta}^{p-1}\right) \cap B_{\varepsilon}^{n} \rightarrow S_{\delta}^{p-1}, \\
\mathrm{e} \\
g: g^{-1}\left(S_{\delta}^{p-2}\right) \cap B_{\varepsilon}^{n} \rightarrow S_{\delta}^{p-2} .
\end{gathered}
$$

Em [39, p. 100] Milnor conjecturou o seguinte.

Conjectura 2.3.1. A fibra da fibração associada com essa nova aplicação é homeomorfa ao produto da fibra antiga com o intervalo unitário.

Em [16] os autores R. Araújo dos Santos e N. Dutertre obtiveram resultados que valem em um contexto mais geral, o qual inclui o caso de singularidades não-isoladas. Contudo, 
no caso especial de uma singularidade isolada, o resultado enunciado a seguir fornece uma resposta positiva à conjectura acima.

Teorema 2.3.2 ([16]). Seja $f:\left(\mathbb{R}^{n}, 0\right) \rightarrow\left(\mathbb{R}^{p}, 0\right), n>p \geqslant 2$, um germe de aplicação polinomial satisfazendo as condições $(a)$ e $(b)$ de Milnor na origem e considere $G=\pi \circ f$ : $\left(\mathbb{R}^{n}, 0\right) \rightarrow\left(\mathbb{R}^{p-1}, 0\right)$. Então, a fibra de Milnor ${ }^{1} F_{G}$ de $G$ é homeomorfa a $F_{f} \times[0,1]$. Em particular, $F_{G}$ e $F_{f}$ têm o mesmo tipo de homotopia.

Este teorema será muito importante para a obtenção de novos resultados no Capítulo 5.

Como apontado por Milnor em [39, p. 100], considerar um germe de aplicação polinomial $f:\left(\mathbb{R}^{n}, 0\right) \rightarrow\left(\mathbb{R}^{p}, 0\right)$ com singularidade isolada na origem como hipótese para o Teorema 2.1.7 é tão forte que é difícil encontrar exemplos. Assim, ele colocou a seguinte questão.

Problema 2.3.3. Para quais dimensões $n \geqslant p \geqslant 2$ existem exemplos não-triviais?

Na verdade, Milnor não especificou o que significa uma "aplicação trivial", mas em um certo sentido ele propôs chamar uma aplicação polinomial com singularidade isolada de "trivial" quando a fibra $F_{f}$ da fibração obtida no Teorema 2.1.7 for difeomorfa ao disco fechado $(n-p)$-dimensional. Por exemplo, em vista do Lema 2.2.4, dado um germe de aplicação polinomial $f:\left(\mathbb{R}^{4}, 0\right) \rightarrow\left(\mathbb{R}^{3}, 0\right)$ com singularidade isolada e com link não vazio, temos que a aplicação é trivial. De fato, sendo a fibra uma variedade 1-dimensional com bordo e 1-conexa, ela tem que ser difeomorfa ao intervalo $[-1,1]$.

Com esta definição, Church e Lamotke em [12, pp.149-150], responderam a questão acima da seguinte forma.

Teorema 2.3.4. (Versão atualizada após G. Perelman) (a) Para $0 \leqslant n-p \leqslant 2$, exemplos não-triviais ocorrem precisamente para as dimensões $(n, p) \in\{(2,2),(4,3),(4,2)\}$.

(b) Para $n-p \geqslant 4$, exemplos não-triviais ocorrem para todo $(n, p)$.

(c) Para $n-p=3$, exemplos não-triviais ocorrem para $(5,2)$, $(8,5)$ e possivelmente $(6,3)$.

Além disso, em [12, p.151], os autores encontraram os pares de dimensões $(n, p)$ para os quais 0 é um ponto isolado em $f^{-1}(0)$, i.e., localmente $f^{-1}(0)=\{0\}$. Isto equivale ao link $K_{\varepsilon}=f^{-1}(0) \cap S_{\varepsilon}^{n-1}$ ser vazio. Eles provaram o seguinte.

Lema 2.3.5. ([12, Lema 1, p.151]) Se 0 é um ponto isolado de $f^{-1}(0)$, então $n=p$, ou $(n, p) \in\{(4,3),(8,5),(16,9)\}$. Se $n=p$, a singularidade é trivial exceto para $p=2$. Os outros casos nunca são triviais.

\footnotetext{
${ }^{1}$ Se $p=2$, então a fibra de Milnor de $G$ é, por definição, a pré-imagem de um valor regular suficientemente próximo da origem dentro de uma pequena bola fechada centrada na origem de $\mathbb{R}^{n}$.
} 
Ideia da prova. Para $n-p \geqslant 1$, aplique o fato que toda fibra conexa de um fibrado $S^{n} \rightarrow B$, com $B$ conexo e $B \neq$ ponto, é equivalente homotópica à 1-, 3- ou 7-esfera (ver [10]). O resultado segue ao aplicar a sequência de Wang (Proposição 1.2.12). Para $n=p$, basta usar o fato que o fibrado é um recobrimento. No caso $p=2$, a aplicação $f(z)=z^{k}, k>1$ é não-trivial. No caso $p \geqslant 3$, todos os exemplos são triviais pois cada fibra consiste de um único ponto pelo fato de sua cardinalidade ser igual à cardinalidade do grupo fundamental do espaço base.

Na demonstração do Teorema 2.3.4, feita pelos autores, o caso $(6,3)$ foi omitido.

Ideia da prova do Teorema 2.3.4 conforme [12]. (a) Supondo $0<n-p \leqslant 2$ e $K \neq \emptyset$, temos que a fibra $(n-p)$-dimensional é $(p-2)$-conexa (de acordo com o Lema 2.2.4) e pela classificação de variedades nessas dimensões (ver [38] e [36]), concluímos que os exemplos são sempre triviais, exceto para o par $(4,2)$, pois existem polinômios complexos em duas variáveis que fornecem exemplos não-triviais, como por exemplo $f(z, w)=z^{2}+w^{3}$, cuja fibra é um toro com um disco aberto removido. Para $K=\emptyset$ e $0 \leqslant n-p \leqslant 2$, o resultado segue do Lema 2.3.5, ou seja, é possível encontrar exemplos não-triviais nos pares de dimensões $(2,2)$ e $(4,3)$.

(b) A partir de uma variedade $(n-p)$-dimensional contrátil, com bordo não simplesmente conexo, prova-se a existência de um NS-par $\left(S^{n-1}, K^{n-p-1}\right)$ com $K$ não simplesmente conexo e o resultado segue ao aplicarmos o Teorema 2.4.4.

(c) Caso o link seja não-vazio e $n-p=3 \operatorname{com} p \geqslant 3$, temos, pelo Lema 2.2.4, que a fibra é simplesmente conexa e além disso, é contrátil se $p \geqslant 4$. Pelo Lema 3.0.7, para $p \geqslant 4$, a fibra é difeomorfa a um disco, logo os exemplos são triviais. Dado um exemplo não-trivial no par $(4,2)$, ele fornece um exemplo não-trivial no par $(5,2)$ usando o Spinning ${ }^{2}$, conforme observação feita por Looijenga em [34]. Caso o link seja vazio, pelo Lema 2.3.5, existe exemplo não-trivial no par $(8,5)$. Não temos como garantir a existência de exemplos nãotriviais no par $(6,3)$ por não termos mais informações sobre a topologia da fibra. Mas se o bordo da fibra não for uma 2-esfera de homologia, então a fibra não é contrátil e consequentemente não é difeomorfa ao disco.

Observe que, como a Conjectura de Poincaré para variedade de dimensão 3 foi provada positivamente, tem-se que a aplicação $f$, com $n-p=3$, pode ser não-trivial apenas se $(n, p) \in\{(6,3),(8,5),(5,2)\}$. Portanto, o Problema 2.3.3 está aberto unicamente para o par de dimensões $(6,3)$.

\footnotetext{
${ }^{2}$ Construção que será apresentada na próxima seção.
} 
Ainda em [12], encontramos uma caracterização alternativa (ver a Proposição 2.3.8 a seguir) de germe de aplicações triviais usando o conjunto de bifurcação $B_{f}$ de um germe de aplicação $f$, isto é, o conjunto no qual o germe de aplicação não é localmente topologicamente equivalente a uma projeção. Decorre da definição e do Teorema da Função Inversa que se $x \notin \Sigma_{f}$ então $x \notin B_{f}$, portanto $B_{f} \subseteq \Sigma_{f}$.

Antes de enunciarmos e provarmos tal resultado, vamos relembrar algumas definições.

Definição 2.3.6. Dizemos que uma aplicação $g: X \rightarrow Y$ em $x \in X$ é localmente topologicamente equivalente a uma aplicação $g^{\prime}: X^{\prime} \rightarrow Y^{\prime}$ em $x^{\prime} \in X^{\prime}$ se existem vizinhanças abertas $U$ de $x, U^{\prime}$ de $x^{\prime}, V$ de $g(x)$, e $V^{\prime}$ de $g^{\prime}\left(x^{\prime}\right)$ e homeomorfismos $\alpha: U \rightarrow U^{\prime}$ com $\alpha(x)=x^{\prime}$ e $\beta: V \rightarrow V^{\prime}$ tais que $\beta \circ g_{\mid U}=g^{\prime} \circ \alpha$.

Definição 2.3.7. ([31]) Uma aplicação completamente regular $p$ de um espaço métrico $(E, \ell)$, onde $\ell$ é uma métrica em $E$, sobre um espaço métrico conexo $(B, d)$ é uma aplicação tal que para cada $b \in B$ e $\mu>0$, existe um $\delta=\delta(b, \mu)>0$ tal que se $b^{\prime} \in B$ com $d\left(b, b^{\prime}\right)<\delta$, então existe um homeomorfismo $h: p^{-1}(b) \rightarrow p^{-1}\left(b^{\prime}\right)$ tal que $\ell(x, h(x))<\mu$ para cada $x \in p^{-1}(b)$.

Além disso, o autor S. Kim mostrou, em [31], que no caso de $B$ ser contrátil e a fibra ser uma variedade compacta (possivelmente com bordo), a aplicação completamente regular $p: E \rightarrow B$ é um fibrado trivial.

Proposição 2.3.8. ([12]) Para $n-p \neq 4,5, f$ é trivial se, e somente se, $0 \notin B_{f}$.

Demonstração. $\Rightarrow$ ] Suponha que a fibra F (fibra da fibração (2.1.1), página 24) é difeomorfa à bola $B^{n-p}$.

Pelo resultado de Kim citado anteriormente e pela definição de $B_{f}$, é suficiente provar que a aplicação

$$
B_{\varepsilon}^{n} \cap f^{-1}\left(B_{\delta}^{p}\right) \rightarrow B_{\delta}^{p}
$$

é completamente regular, para $\varepsilon$ e $\delta$ satisfazendo o Teorema 2.1.2 (ver também a Proposição 2.1.5).

Mostraremos que a condição de regularidade completa se estende para o fibrado (2.3.1). Pelo Teorema 2.1.2, a projeção $f_{\mid}: B_{\varepsilon}^{n} \cap f^{-1}\left(\stackrel{\circ}{B}_{\delta}^{p} \backslash\{0\}\right) \rightarrow \stackrel{\circ}{B}_{\delta}^{p} \backslash\{0\}$ é completamente regular. Dado $\mu>0$, escolha $0<\rho<\min (\mu / 2, \varepsilon)$, e $\zeta>0$ satisfazendo o Lema 2.1.6 para $\varepsilon$ e $\rho$, de modo que

$$
B_{\rho}^{n} \cap f^{-1}\left(B_{\zeta}^{p} \backslash\{0\}\right) \rightarrow B_{\zeta}^{p} \backslash\{0\}
$$

é um fibrado. Ainda pelo Lema 2.1.6, existe um homeomorfismo

$$
\left(B_{\varepsilon}^{n} \backslash \operatorname{Int} B_{\rho}^{n}\right) \cap f^{-1}(y) \stackrel{\beta}{\rightarrow}\left(B_{\varepsilon}^{n} \backslash \operatorname{Int} B_{\rho}^{n}\right) \cap f^{-1}(0)
$$


para cada $y \in B_{\zeta}^{p} \backslash\{0\}$, satisfazendo $\beta\left(S_{\rho}^{n-1} \cap f^{-1}(y)\right)=S_{\rho}^{n-1} \cap f^{-1}(0)$, ou seja, $\beta(\partial F)=K$. Para $\zeta$ suficientemente pequeno, temos que $d(x, \beta(x))<\mu$ para todo $x$ em $\left(B_{\varepsilon}^{n} \backslash \operatorname{Int} B_{\rho}^{n}\right) \cap$ $f^{-1}(y)$. Lembre que, por hipótese, dentro da bola $B_{\rho}^{n}$, a fibra $F$ é difeomorfa à $B^{n-p}, \operatorname{logo}$ $K \approx \partial F \approx S^{n-p-1}$. Por outro lado, ainda dentro da bola $B_{\rho}^{n}$, temos que a fibra $f^{-1}(0)$ é o cone sobre $K$ (ver [39, Proposição 2.10]), e consequentemente, é homeomorfa à bola $B^{n-p}$. Dessa forma, podemos estender $\beta$ para um homeomorfismo

$$
B_{\varepsilon}^{n} \cap f^{-1}(y) \stackrel{\gamma}{\rightarrow} B_{\varepsilon}^{n} \cap f^{-1}(0)
$$

satisfazendo $\gamma\left(B_{\rho}^{n} \cap f^{-1}(y)\right)=B_{\rho}^{n} \cap f^{-1}(0)$. Como o diâmetro da bola $B_{\rho}^{n}$ é menor que $\mu$ (por escolha), temos que $d(x, \gamma(x))<\mu$ para todo $x \in B_{\rho}^{n} \cap f^{-1}(y)$ e assim, obtemos o resultado desejado, o qual segue para todo $n-p$.

$\Leftarrow]$ Suponha que a aplicação $f$ na origem seja localmente topologicamente equivalente à aplicação projeção $p: \mathbb{R}^{n} \rightarrow \mathbb{R}^{p}$ na origem. Então, por definição, considerando $\varepsilon$ e $\delta$ satisfazendo o Teorema 2.1.2, existem uma vizinhança $0 \in U \subset B_{\varepsilon}^{n} \cap f^{-1}\left(B_{\delta}^{p}\right)$ e um homeomorfismo $U \stackrel{\alpha}{\rightarrow} B^{n-p} \times B_{\xi}^{p}$, para algum $\xi$ com $0<\xi<\delta$, tais que a composição

$$
U \stackrel{\alpha}{\rightarrow} B^{n-p} \times B_{\xi}^{p} \stackrel{\pi}{\rightarrow} B_{\xi}^{p}
$$

é a restrição de $f$, onde $\pi$ é a projeção. Agora, considerando $0<\zeta<\xi$ e $\rho>0$ como no Lema 2.1.6 de modo que $B_{\rho}^{n} \subset U$, temos, para $y \in B_{\zeta}^{p} \backslash\{0\}$

$$
B_{\rho}^{n} \cap f^{-1}(y) \subset U \cap f^{-1}(y) \subset B_{\varepsilon}^{n} \cap f^{-1}(y) .
$$

Analisando esta composição, vemos que, por (2.3.2), a fibra $f^{-1}(y)$ na vizinhança $U$ é homeomorfa à bola $B^{n-p}$. Além disso, os termos nas extermidades são homeomorfos à fibra $F$. Pelo Lema 2.1.6, $\left(B_{\varepsilon}^{n} \backslash \operatorname{Int} B_{\rho}^{n}\right) \cap f^{-1}(y)$ é homeomorfo a $K \times I$, o que implica que cada inclusão $B_{\rho}^{n} \cap f^{-1}(y) \hookrightarrow B_{\varepsilon}^{n} \cap f^{-1}(y)$ é uma equivalência de homotopia. Assim, $\pi_{i}\left(B_{\varepsilon}^{n} \cap f^{-1}(y)\right) \cong \pi_{i}\left(B^{n-p}\right)=0$ para todo $i$ e consequentemente, $F$ (que é homeomorfa a $\left.B_{\varepsilon}^{n} \cap f^{-1}(y)\right)$ é contrátil.

Como $\pi \circ \alpha: U \approx B^{n-p} \times B_{\xi}^{p} \rightarrow B_{\xi}^{p}$ coincide com $f_{\left.\right|_{U}}: U \rightarrow B_{\xi}^{p}$, temos $f^{-1}(0) \cap U \approx$ $B^{n-p} \times\{0\}$. Portanto, $f^{-1}(0)$ é uma variedade topológica $(n-p)$-dimensional na origem.

Além disso, consideramos a sequência

$$
B_{\rho}^{n} \cap f^{-1}(0)-\{0\} \subset U \cap f^{-1}(0)-\{0\} \subset B_{\varepsilon}^{n} \cap f^{-1}(0)-\{0\} .
$$

A composição inclusão é uma equivalência de homotopia pelo Lema 2.1.6. Além disso, considerando $U^{\prime} \approx B^{n-p} \times B_{\zeta}^{p}$ apropriadamente dentro de $U$, temos que a composição inclusão

$$
U^{\prime} \cap f^{-1}(0)-\{0\} \subset B_{\rho}^{n} \cap f^{-1}(0)-\{0\} \subset U \cap f^{-1}(0)-\{0\}
$$


é também uma equivalência de homotopia. Combinando essas duas, vemos que a inclusão

$$
B_{\rho}^{n} \cap f^{-1}(0)-\{0\} \subset U \cap f^{-1}(0)-\{0\}
$$

induz isomorfismos nos grupos de homotopia em todas as dimensões, e assim, é uma equivalência de homotopia. Como o último espaço é equivalente homotópico a $S^{n-p-1}$ e o primeiro espaço é equivalente homotópico a $K$, temos que $K$ é uma $(n-p-1)$-esfera de homotopia.

Se $n-p \leqslant 3$, então, pela classificação das variedades nessas dimensões, temos que $F$ é difeomorfa à $B^{n-p}$. Pela Proposição 1.2.23, obtemos a mesma conclusão para o caso $n-p \geqslant 6$. Portanto $f$ é trivial para $n-p \neq 4,5$.

Em [47], usamos a Proposição 1.3.3 para mostrarmos uma caracterização mais "manejável" do Teorema 2.3.4 (de Church-Lamotke) quando a fibra de Milnor é 3-dimensional. Esta caracterização será apresentada e provada no Capítulo 3.

\subsection{Nós, links e NS-pares}

Um subconjunto $K$ de um espaço $X$ é um nó se $K$ é homeomorfo à esfera $S^{p}$ e, neste caso, a dimensão do nó $K$ é definida como sendo a dimensão da esfera $S^{p}$. Mais geralmente, $K$ é um link se ele é homeomorfo à união disjunta $S^{p_{1}} \cup \cdots \cup S^{p_{r}}$ de esferas. Dessa forma, um nó pode ser descrito como um link com uma componente. Dois nós ou links $K, K^{\prime}$ são equivalentes se existe um homeomorfismo $h: X \rightarrow X$ tal que $h(K)=K^{\prime}$.

Como trabalharemos com nós de dimensões maiores que ou iguais a 1, muitas vezes escreveremos $n$-nó para representarmos um nó de dimensão $n$.

Definição 2.4.1. Um nó ou link $L^{n}$ em $S^{n+2}$ é fibrado se existe uma fibração $f: S^{n+2} \backslash$ $L^{n} \rightarrow S^{1}$, além disso, cada componente $L_{i}$ deve admitir uma vizinhança difeomorfa à $S^{n} \times D^{2}$, com $L_{i} \approx S^{n} \times 0$, de tal forma que a restrição $f_{\left.\right|_{S^{n} \times\left(D^{2} \backslash\{0\}\right)}}: S^{n} \times\left(D^{2} \backslash\{0\}\right) \rightarrow S^{1}$ seja dada por $(x, y) \mapsto y /|y|$.

Em 1925 Emil Artin criou dois métodos, em [7], para construir 2-nós em $\mathbb{R}^{4}$ a partir de um nó clássico. Um dos métodos é conhecido por spinning, o qual usa um processo de rotação e tem desempenhado papéis importantes no estudo de superfícies nodadas. Mais tarde, em 1965, Zeeman generalizou a construção de Artin com o método chamado twist spinning (ver [51]). A seguir veremos um pouco destas duas construções. 


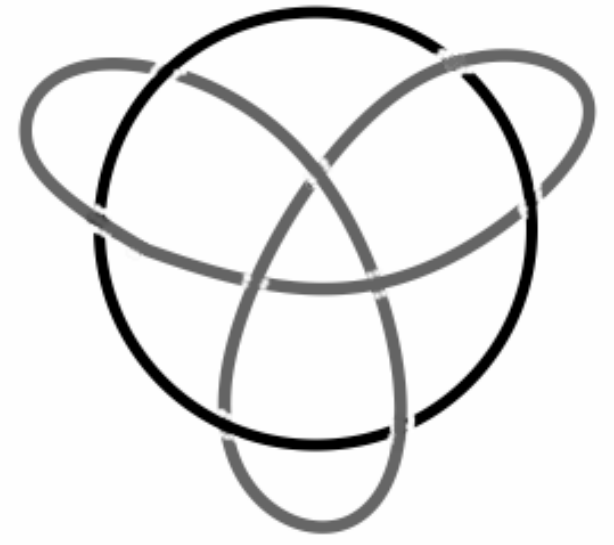

Figura 2.4: Link com duas componentes: o nó trefoil e o círculo

\section{Spinning}

Considere, em $\mathbb{R}^{4}$, os subconjuntos a seguir.

$$
\mathbb{R}_{+}^{3}=\left\{\left(x_{1}, x_{2}, x_{3}, x_{4}\right) \mid x_{3} \geqslant 0, x_{4}=0\right\} \text { e } \mathbb{R}^{2}=\left\{\left(x_{1}, x_{2}, 0,0\right)\right\}
$$

Pode-se rotacionar qualquer ponto $x=\left(x_{1}, x_{2}, x_{3}, 0\right)$ de $\mathbb{R}_{+}^{3}$ em torno de $\mathbb{R}^{2}$ de acordo com a fórmula $x_{\theta}=\left(x_{1}, x_{2}, x_{3} \cos \theta, x_{3} \sin \theta\right)$.

Defina o spin, $\tilde{X}$, de um conjunto $X \subset \mathbb{R}_{+}^{3}$ como sendo $\tilde{X}=\left\{x_{\theta} ; x \in X, 0 \leqslant \theta \leqslant 2 \pi\right\}$.

Para obter um nó em $\mathbb{R}^{4}$, escolha um arco nodado $\alpha$ mergulhado no semi-espaço 3dimensional $\mathbb{R}_{+}^{3}$ com extremidades em $\mathbb{R}^{2}$ (ver figura a seguir, a qual representa a mesma ideia de [42, p.85]). Então ã é um 2-nó em $\mathbb{R}^{4}$, chamado um nó spun.
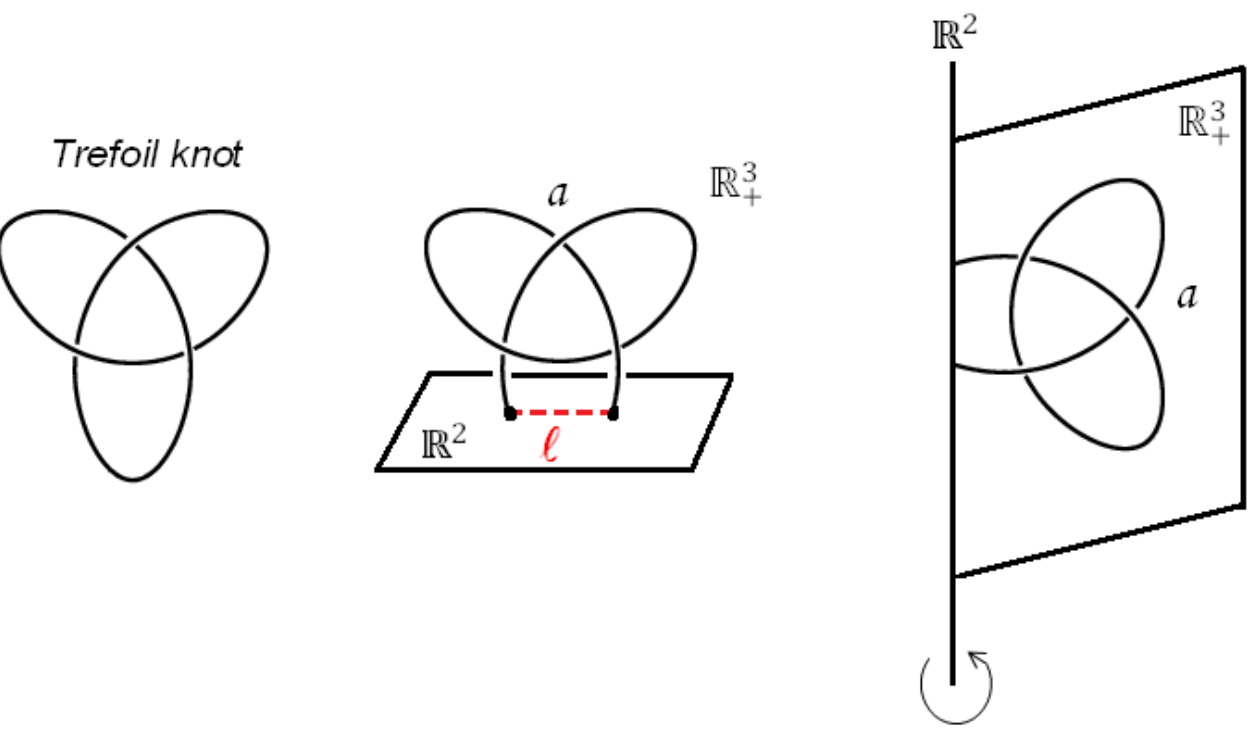
Observações 2.4.2. (a) O grupo do nó $\tilde{\alpha}, \pi_{1}\left(\mathbb{R}^{4} \backslash \tilde{\alpha}\right)$, é isomorfo a $\pi_{1}\left(\mathbb{R}_{+}^{3} \backslash \alpha\right)$ o qual, por sua vez, é isomorfo ao grupo do nó associado ${ }^{3}$ ao arco nodado $\alpha$.

(b) O spun do nó fibrado é também um nó fibrado (ver detalhes na Seção 5.1)

\section{Twist spinning}

Novamente começamos com um nó 1-dimensional e obtemos um nó 2-dimensional. A diferença é que este método usa, além da rotação, um processo de "torção" enquanto o nó é rotacionado. Mais precisamente podemos colocar a parte nodada de $\alpha$ em uma bola 3 -dimensional e "torcer" ela $k$ vezes (ou rotacionar $k$ vezes em torno de seu eixo) enquanto aplica o spinning em torno do $\mathbb{R}^{2}$. Um outro 2-nó é obtido em $\mathbb{R}^{4}$ e ele é chamado $k$-twist spun.

A figura a seguir representa apenas um half-twist.
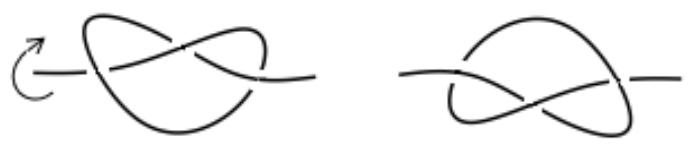

\section{A construção de Looijenga}

Inicialmente veremos uma definição dada por Looijenga em [34].

Definição 2.4.3. Considere $K=K^{n-p-1}$ uma subvariedade orientada $(n-p-1)$ dimensional da esfera orientada $S^{n-1}$ com fibrado normal trivial, ou considere $K=\emptyset$. Suponha que para alguma trivialização $c: N(K) \rightarrow K \times D^{p}$ de uma vizinhança tubular $N(K)$ de $K$, o fibrado definido pela composição

$$
N(K) \backslash K \stackrel{c}{\rightarrow} K \times\left(D^{p} \backslash\{0\}\right) \stackrel{\pi}{\rightarrow} S^{p-1}
$$

com a última projeção dada por $\pi(x, y)=y /\|y\|$, se estende para um fibrado suave $S^{n-1} \backslash K \rightarrow S^{p-1}$. Então, o par $\left(S^{n-1}, K^{n-p-1}\right)$ é chamado um Neuwirth-Stallings par, ou de forma abreviada, um NS-par.

Note que a definição de NS-par estende a definição de link fibrado para $p \geqslant 2$.

De acordo com o Teorema 2.1.7 e com a definição acima, para todo $\varepsilon>0$ suficientemente pequeno, o par $\left(S_{\varepsilon}^{n-1}, f^{-1}(0) \cap S_{\varepsilon}^{n-1}\right)$ é um NS-par. Neste caso, Looijenga o chamou de NS-par associado à singularidade.

\footnotetext{
${ }^{3} \mathrm{Um}$ nó é associado a um arco nodado se ele é obtido da conexão das extremidades do arco nodado com um arco em $\mathbb{R}^{2}$.
} 
Como mencionado anteriormente, em [6] os autores consideraram um germe de aplicação analítica real $f:\left(\mathbb{R}^{n}, 0\right) \rightarrow\left(\mathbb{R}^{p}, 0\right)$ com conjunto singular satisfazendo $\Sigma_{f} \cap V \subseteq\{0\}$ e mostraram que o par $\left(S_{\varepsilon}^{n-1}, f^{-1}(0) \cap S_{\varepsilon}^{n-1}\right)$ é um NS-par com projeção do fibrado dada por $f /\|f\|: S_{\varepsilon}^{n-1} \backslash K \rightarrow S^{p-1}$, onde $K=f^{-1}(0) \cap S_{\varepsilon}^{n-1}$ é uma variedade suave. Ela foi chamada uma estrutura open book superior da esfera $S_{\varepsilon}^{n-1}$. Em [2, 3] foi mostrada uma extensão de tais estruturas para esferas de raio suficientemente pequeno e raio suficientemente grande (no infinito), mas permitindo singularidade no "binding" $K$. Neste caso, ela foi chamada uma estrutura open book singular da esfera.

Looijenga mostrou como usar a soma conexa de NS-pares para a construção de novos NS-pares. A seguir daremos uma ideia dessa construção. Para mais detalhes pode-se consultar [34, p. 419].

Dada uma variedade orientada $M$, Looijenga denotou, por convenção, a mesma variedade com a orientação invertida por $-M$. Além disso, ele denotou por $(-1)^{k} M$ a mesma variedade $M$, significando que para $k$ par, a orientação é preservada e para $k$ ímpar, a orientação é invertida.

Dado um NS-par $\left(S^{n}, K^{n-p-1}\right)$ com fibra $F$, onde $K=\cup_{i=1}^{k} K_{i}$, tome um ponto $p$ em uma determinada componente $K_{i}$ de $K^{n-p-1}$ e considere uma pequena bola aberta centrada em $p$ em $S^{n}$ de tal forma que sua interseção com $K_{i}$ é um disco aberto $\stackrel{\circ}{D}^{n-p-1}$ (ver a próxima figura). Remova tal disco. Agora considere um NS-par $\left((-1)^{n-1} S^{n},(-1)^{n-p} K^{n-p-1}\right)$ com fibra $(-1)^{n-p} F$ e faça o procedimento análogo ao anterior. Assim, identificando os bordos resultantes obtemos as variedades $S^{n} \sharp(-1)^{n-1} S^{n}$ e $K^{n-p-1} \sharp(-1)^{n-p} K^{n-p-1}$.
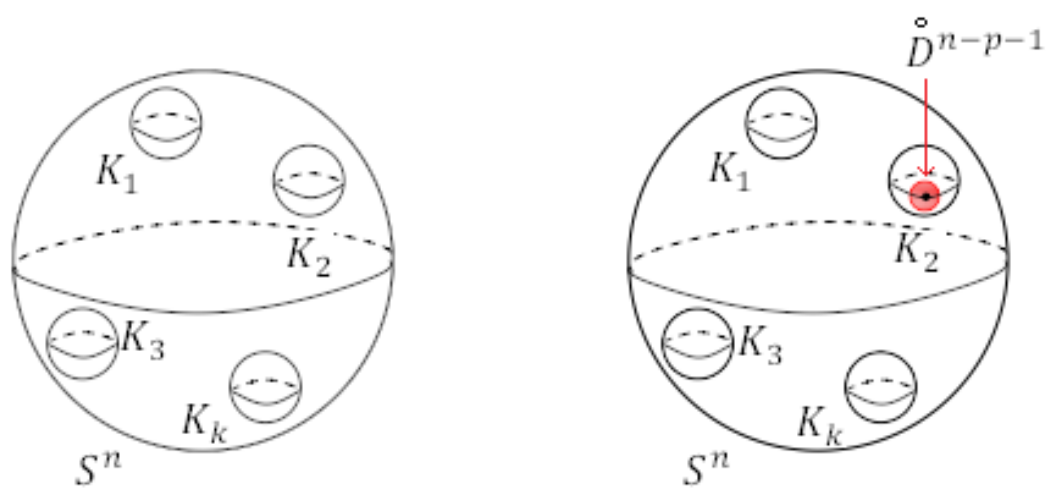

Além disso, Looijenga mostrou que o par $\left(S^{n} \sharp(-1)^{n-1} S^{n}, K^{n-p-1} \sharp(-1)^{n-p} K^{n-p-1}\right)$ é um NS-par com fibra difeomorfa ao interior de $\bar{F} \natural(-1)^{n-p} \bar{F}$ (onde " $\downarrow$ " significa a soma conexa ao longo do bordo) e o denotou por

$$
\left(S^{n}, K^{n-p-1}\right) \sharp\left((-1)^{n-1} S^{n},(-1)^{n-p} K^{n-p-1}\right) .
$$


Decorre da construção que, como $K^{n-p-1}$ tem $k$ componentes disjuntas, então a variedade $K^{n-p-1} \sharp(-1)^{n-p} K^{n-p-1}$ tem exatamente $2 k-1$ componentes conexas.

O teorema a seguir é o principal resultado em [34]:

Teorema 2.4.4. Seja $\left(S^{n}, K^{n-p-1}\right)$ um $N S$-par com $K \neq \emptyset$. Então existe um germe de aplicação polinomial com singularidade isolada $\left(\mathbb{R}^{n+1}, 0\right) \rightarrow\left(\mathbb{R}^{p+1}, 0\right)$ cujo NS-par associado é isomorfo a $\left(S^{n}, K\right) \sharp\left((-1)^{n-1} S^{n},(-1)^{n-p} K\right)$.

\subsection{Linking number e espaço de configuração}

Dadas variedades disjuntas, $M, N \subset \mathbb{R}^{k+1}$, considere a aplicação $\lambda: M \times N \rightarrow S^{k}$ definida por

$$
\lambda(x, y)=\frac{(x-y)}{\|x-y\|} .
$$

Se $M$ e $N$ são compactas, orientadas e sem bordo, com dimensão total $m+n=k$, então o grau de $\lambda$ é chamado o linking number $\operatorname{lk}(M, N)$.

Também temos que $\operatorname{lk}(N, M)=(-1)^{(m+1)(n+1)} \operatorname{lk}(M, N)$.

Definição 2.5.1. O espaço de configuração de $k$ pontos na variedade suave $M$ é o espaço

$$
\mathbb{F}_{k}(M)=\left\{\left(x_{1}, x_{2}, \ldots, x_{k}\right) \mid x_{i} \neq x_{j}, i \neq j\right\} \subset \underbrace{M \times \cdots \times M}_{k \text { vezes }} .
$$

Para toda aplicação f : $S^{n-1} \rightarrow \mathbb{F}_{k}\left(\mathbb{R}^{n}\right)$ associamos $k$ mergulhos disjuntos $f_{j}: S^{n-1} \rightarrow$ $S^{n-1} \times B^{n}$ pela fórmula $f_{j}(x)=\left(x, \mathbf{f}(x)_{j}\right)$, onde $\mathbf{f}(x)_{j}$ é a $j$-ésima componente da $k$-upla $\mathbf{f}(x) \in \mathbb{F}_{k}\left(\mathbb{R}^{n}\right)$, e identificamos $\operatorname{int}\left(B^{n}\right) \operatorname{com} \mathbb{R}^{n}$.

Lema 2.5.2. ([20], Lema 2.31) Existe um isomorfismo $\pi_{n-1}\left(\mathbb{F}_{k}\left(\mathbb{R}^{n}\right)\right) \rightarrow \mathbb{Z}^{k(k-1) / 2}$ que associa a classe da aplicação $\mathbf{f}: S^{n-1} \rightarrow \mathbb{F}_{k}\left(\mathbb{R}^{n}\right)$ à matriz $(-1)^{n}$-simétrica cujas entradas da parte triangular superior são dadas por

$$
\left(\operatorname{lk}\left(f_{i}\left(S^{n-1}\right), f_{j}\left(S^{n-1}\right)\right)\right)_{1 \leqslant i<j \leqslant k}
$$




\section{Caracterização de aplicações triviais com fibras de Milnor 3-dimensionais}

Ao longo deste capítulo concentramos o estudo topológico sobre a fibração no "tubo de Milnor", isto é, a fibração

$$
f_{\mid}: f^{-1}\left(S_{\delta}^{p-1}\right) \cap B_{\varepsilon}^{n} \rightarrow S_{\delta}^{p-1}
$$

O nosso objetivo, aqui, é usar ferramentas da Topologia Algébrica, Teoria de Singularidades e uma fórmula para a característica de Euler da fibra de Milnor real (ver Proposição 1.3.3), para estabelecer uma (nova) caracterização de germes de aplicações que são triviais no sentido proposto por Milnor. Ressaltamos que tais resultados foram considerados na tese de doutorado de T. O. Souza (ver detalhes em [46]). Como vimos anteriormente, segue do Teorema 2.3.4 que no caso $n-p=3$, a aplicação singular $f$ pode ser não-trivial apenas se $(n, p) \in\{(6,3),(8,5),(5,2)\}$. Estes casos são cobertos pelo teorema abaixo, como segue.

Teorema 3.0.3. ([47, Teorema 1.6]) Seja $f:\left(\mathbb{R}^{n}, 0\right) \rightarrow\left(\mathbb{R}^{p}, 0\right), f(x)=\left(f_{1}(x), \ldots, f_{p}(x)\right)$ um germe de aplicação polinomial com singularidade isolada, e assuma $n-p=3$.

(a) Se o par $(n, p)=(6,3)$, então são equivalentes:

(i) f é trivial;

(ii) $\operatorname{deg}_{0}\left(\nabla f_{1}\right)=0$;

(iii) $O \operatorname{link} K_{\varepsilon}$ é conexo.

(b) Se o par $(n, p)=(8,5)$, então são equivalentes:

(i) f é trivial;

(ii) $\operatorname{deg}_{0}\left(\nabla f_{1}\right)=0$; 
(iii) $O \operatorname{link} K_{\varepsilon}$ é não-vazio.

(c) Se o par $(n, p)=(5,2)$, então são equivalentes:

(i) $f$ é trivial;

(ii) $\pi_{1}\left(F_{f}\right)=0$, i.e. a fibra de Milnor $F_{f}$ é simplesmente conexa.

Ressaltamos que em [4], seção 4, os autores provaram algumas fórmulas com o objetivo de descrever, de forma geométrica e topológica, a fibra de Milnor real e fornecer uma caracterização de germe de aplicação trivial para o par $(4,2)$. Para isso, eles usaram uma condição análoga à do Teorema 3.0.3 (a), (vide [4, Corolário 4.5]). Eles provaram que um germe de aplicação polinomial com singularidade isolada $f=\left(f_{1}, f_{2}\right):\left(\mathbb{R}^{4}, 0\right) \rightarrow\left(\mathbb{R}^{2}, 0\right)$ é trivial se, e só se, $\operatorname{deg}_{0}\left(\nabla f_{1}\right)=0$.

Para efeito de completitude, vamos estabelecer alguns resultados necessários para relembrarmos a prova do Teorema 3.0.3. O resultado a seguir é um fato bem conhecido da Topologia Algébrica. A prova segue da propriedade aditiva da característica de Euler (veja também [50, Teorema 6.38, p.180]).

Teorema 3.0.4. Se $M$ é uma n-variedade topológica compacta com bordo $\partial M$, então a característica de Euler de $\partial M$ é par. Mais precisamente,

$$
\chi(\partial M)= \begin{cases}2 \chi(M), & \text { se } n \text { é impar } \\ 0, & \text { se } n \text { é par. }\end{cases}
$$

A seguir veremos um outro resultado para variedades com bordo, o qual é uma generalização do teorema da dualidade de Poincaré.

Teorema 3.0.5. (Dualidade de Poincaré-Lefschetz - [48, p.298]) Seja X uma n-variedade compacta com bordo $\partial X$ e orientação $U$. Para todo $q$ existem isomorfismos (onde $j$ : $X-\partial X \hookrightarrow X)$

$$
\begin{gathered}
H_{q}(X ; \mathbb{Z}) \stackrel{j_{*}}{\cong} H_{q}(X-\partial X ; \mathbb{Z}) \stackrel{\cong}{\rightrightarrows} H^{n-q}(X, \partial X ; \mathbb{Z}), \\
H_{q}(X, \partial X ; \mathbb{Z}) \stackrel{\cong}{\rightrightarrows} H^{n-q}(X-\partial X ; \mathbb{Z}) \stackrel{j^{*}}{\cong} H^{n-q}(X ; \mathbb{Z}) .
\end{gathered}
$$

Agora, vamos relembrar alguns resultados relativos à topologia de variedades compactas 3-dimensionais, como segue.

Lema 3.0.6. Seja $M$ uma variedade topológica 3-dimensional, compacta, conexa e orientável com bordo $\partial M$. Se $H_{1}(M ; \mathbb{Z})=0$, então $\partial M$ é uma união disjunta de 2-esferas. 
Demonstração. Considere o seguinte pedaço da sequência exata longa em cohomologia para o par $(M, \partial M)$ :

$$
\cdots \rightarrow H^{1}(M ; \mathbb{Z}) \rightarrow H^{1}(\partial M ; \mathbb{Z}) \rightarrow H^{2}(M, \partial M ; \mathbb{Z}) \rightarrow \cdots
$$

Sendo $M$ compacta e orientável, pelo teorema da dualidade de Poincaré-Lefschetz temos

$$
H^{2}(M, \partial M ; \mathbb{Z}) \cong H_{1}(M ; \mathbb{Z})=0
$$

Por outro lado, $H^{1}(M ; \mathbb{Z}) \cong \operatorname{Hom}\left(H_{1}(M ; \mathbb{Z}), \mathbb{Z}\right)=0$, portanto, de $(3.0 .2)$ segue que $H^{1}(\partial M ; \mathbb{Z})=0$ e usando a dualidade de Poincaré para a 2-variedade fechada orientável $\partial M$

$$
0=H^{1}(\partial M ; \mathbb{Z}) \cong H_{1}(\partial M ; \mathbb{Z})
$$

Pela classificação padrão de 2-variedades compactas, concluímos que $\partial M$ é uma união disjunta de 2-esferas.

Como uma consequência da Conjectura de Poincaré, temos o seguinte.

Lema 3.0.7. (a) Uma variedade topológica $M$, 3-dimensional, compacta, simplesmente conexa, orientável, com bordo $\partial M \approx S^{2}$ é difeomorfa a um 3-disco. (b) Uma variedade topológica M, 3-dimensional, compacta, simplesmente conexa, orientável, com homologia de um ponto é difeomorfa a um 3-disco.

Demonstração. Para provar (a), considere $N=M \cup_{S^{2}} D^{3}$. Então, $N$ é uma 3-variedade topológica fechada, simplesmente conexa e orientável. Pela Conjectura de Poincaré, $N$ é difeomorfa a uma 3-esfera. Portanto, $M$ é difeomorfa a um 3-disco.

Para a afirmação (b), como $M$ é simplesmente conexa, do Lema 3.0.6, temos que $\partial M$ é uma união disjunta de 2-esferas. Portanto, se $M$ tem a mesma homologia de um ponto, segue que $\chi(M)=1$. Consequentemente, pelo Teorema 3.0.4,

$$
\chi(\partial M)=2 \chi(M)=2,
$$

i.e., $\partial M$ é uma 2-esfera e o resultado segue da afirmação (a) .

\subsection{Prova do Teorema 3.0.3}

Agora, vamos assumir que $f:\left(\mathbb{R}^{n}, 0\right) \rightarrow\left(\mathbb{R}^{p}, 0\right), n \geqslant p \geqslant 2, f(x)=\left(f_{1}(x), \ldots, f_{p}(x)\right)$ é um germe de aplicação polinomial com singularidade isolada na origem, satisfazendo $n-p=3$. Por simplicidade, dividimos a prova do Teorema 3.0.3 em três partes. A primeira refere-se ao par $(6,3)$. 


\subsubsection{O caso $(6,3)$}

Seja $f:\left(\mathbb{R}^{6}, 0\right) \rightarrow\left(\mathbb{R}^{3}, 0\right)$ satisfazendo as condições anteriores. Do Lema 2.3.5 temos que o link $K_{\varepsilon}$, o qual é difeomorfo ao bordo da fibra de Milnor, $\partial F_{f}$, é não-vazio.

Prova do Teorema 3.0.3, item (a). Se $f$ é trivial, então $F_{f}$ é difeomorfa a um 3-disco. Assim $\chi\left(F_{f}\right)=1$. Uma vez que o item $(i)$ da Proposição 1.3 .3 garante que $\chi\left(F_{f}\right)=$ $1-\operatorname{deg}_{0}\left(\nabla f_{1}\right)$, concluímos que $\operatorname{deg}_{0}\left(\nabla f_{1}\right)=0$.

Por outro lado, sendo $p=3$, a fibra de Milnor $F_{f}$ é simplesmente conexa. Assim, em particular, $H_{1}\left(F_{f} ; \mathbb{Z}\right)=0$. Segue do Lema 3.0.6 que $\partial F_{f}$ é uma união disjunta de 2-esferas. Portanto, se $\operatorname{deg}_{0}\left(\nabla f_{1}\right)=0$, então $\chi\left(F_{f}\right)=1 \mathrm{e}$

$$
\chi\left(K_{\varepsilon}\right)=\chi\left(\partial F_{f}\right)=2 \chi\left(F_{f}\right)=2 .
$$

Logo, o link $K_{\varepsilon}$ é difeomorfo a uma 2-esfera e pelo Lema 3.0.7, temos que $F_{f}$ deve ser difeomorfa a um 3-disco.

A implicação $(i) \Rightarrow$ (iii) é trivial. Para a volta, se assumimos que o link $K_{\varepsilon}$ é conexo, então ele deve ser difeomorfo a uma cópia simples de uma 2-esfera e pelo Lema 3.0.7(a), $F_{f}$ é difeomorfa a um 3-disco.

Observação 3.1.1. A afirmação $(a)($ iii $)$ do Teorema 3.0 .3 não caracteriza, em geral, a fibração trivial. Por exemplo, no caso do par $(4,2)$, se consideramos $f(x, y)=x^{2}+y^{3}$, temos que o link é o $(2,3)$-nó toro que é conexo, mas a fibra $F_{f}$ da fibração de Milnor não é difeomorfa ao 2-disco.

Proposição 3.1.2. Dado um germe de aplicação polinomial $f:\left(\mathbb{R}^{6}, 0\right) \rightarrow\left(\mathbb{R}^{3}, 0\right)$ com uma singularidade isolada na origem, tem-se que a característica de Euler-Poincaré da fibra, $\chi\left(F_{f}\right)$, é precisamente o número de esferas sobre seu bordo $\partial F_{f}$.

Demonstração. Uma vez que $F_{f}$ é uma variedade compacta 3-dimensional, simplesmente conexa e com bordo dado por $d$ cópias disjuntas de 2-esferas, temos que cada esfera sobre $\partial F_{f}$ contribui com 2 para a sua característica de Euler. Portanto,

$$
2 . d=\chi\left(\partial F_{f}\right)=2 \cdot \chi\left(F_{f}\right) \text {. }
$$

Desta forma, a característica de Euler da fibra $F_{f}$ é o número de esferas sobre o seu bordo.

\subsubsection{O caso $(8,5)$}

Vamos considerar $f:\left(\mathbb{R}^{8}, 0\right) \rightarrow\left(\mathbb{R}^{5}, 0\right)$ um germe de aplicação polinomial com singularidade isolada na origem. Segue do Lema 2.3.5 que para o par $(8,5)$ o link pode ser 
vazio.

Prova do Teorema 3.0.3, item (b). Por um argumento similar àquele usado na prova do Teorema 3.0.3(a), segue que a condição $\operatorname{deg}_{0}\left(\nabla f_{1}\right)=0$ também caracteriza a trivialidade de $f$ neste caso.

A implicação $(i) \Rightarrow($ iii $)$ é óbvia. Para a recíproca, sendo o link $K_{\varepsilon}$ não-vazio e $p=5$, a fibra de Milnor $F_{f}$ é uma variedade 3-dimensional orientável, compacta com bordo e 3-conexa (logo, simplesmente conexa). Do Teorema de Hurewicz (Teorema 1.1.7), $F_{f}$ deve ter a homologia de um ponto, e assim, pelo Lema 3.0.7(b), $F_{f}$ é difeomorfa a um 3-disco.

\subsubsection{O caso $(5,2)$}

Agora, vamos considerar $f:\left(\mathbb{R}^{5}, 0\right) \rightarrow\left(\mathbb{R}^{2}, 0\right)$ satisfazendo as condições anteriores. Neste caso, temos apenas que a fibra de Milnor $F_{f}$ é conexa e o link $K_{\varepsilon}$ é não-vazio.

Prova do Teorema 3.0.3, item (c). A primeira implicação é evidente. Para a implicação inversa, se $F_{f}$ é simplesmente conexa, segue do Lema 3.0.6 que $\partial F_{f}=K_{\varepsilon}$ é difeomorfo a uma união disjunta de um número finito de 2-esferas. Por outro lado, como o espaço de origem tem dimensão ímpar, pela Proposição 1.3.3(ii), temos $\chi\left(F_{f}\right)=1$ e assim, pelo Teorema 3.0 .4

$$
\chi\left(K_{\varepsilon}\right)=\chi\left(\partial F_{f}\right)=2 \chi\left(F_{f}\right)=2 .
$$

Portanto, o link $K_{\varepsilon}$ deve ser uma 2-esfera e pelo Lema 3.0.7(a), $F_{f}$ é difeomorfa a um 3-disco.

Observação 3.1.3. Na Seção 2.4, vimos que a construção de Looijenga nos permite usar o exemplo não-trivial $f:\left(\mathbb{C}^{2}, 0\right) \rightarrow(\mathbb{C}, 0), f(x, y)=x^{2}+y^{3}$ (vide [4]) como um germe de aplicação polinomial real de $\left(\mathbb{R}^{4}, 0\right) \rightarrow\left(\mathbb{R}^{2}, 0\right)$ para garantirmos a existência de um germe de aplicação polinomial não-trivial $g:\left(\mathbb{R}^{5}, 0\right) \rightarrow\left(\mathbb{R}^{2}, 0\right)$. Portanto, como uma consequência de nossa caracterização, concluímos que a respectiva fibra de Milnor de $g$ não pode ser simplesmente conexa.

Observação 3.1.4. Do ponto de vista topológico, é provável que a hipótese $\pi_{1}\left(F_{f}\right)=0$ não pode ser enfraquecida pela condição $H_{1}\left(F_{f}\right)=0$ devido ao fato que existe uma variedade compacta 3-dimensional $W$ que é uma 3-esfera de homologia mas que não é simplesmente conexa (exemplo clássico dado por Poincaré). Assim, $W \backslash \operatorname{int} D^{3}$ é uma variedade 3dimensional compacta, cujo bordo é uma 2-esfera, mas que não é simplesmente conexa. Contudo, até o momento, não sabemos se $W \backslash \operatorname{int} D^{3}$ pode ser realizada como a fibra de um germe de aplicação $f:\left(\mathbb{R}^{5}, 0\right) \rightarrow\left(\mathbb{R}^{2}, 0\right)$ com singularidade isolada. 


\section{Novos exemplos de NS-pares}

Neste capítulo, objetivamos caracterizar NS-pares $\left(S^{5}, K\right) \operatorname{com} \operatorname{dim} K=2$, e usar tal caracterização para provar a existência de germes de aplicações polinomiais reais nãotriviais $\left(\mathbb{R}^{6}, 0\right) \rightarrow\left(\mathbb{R}^{3}, 0\right)$ com uma singularidade isolada na origem, colocando um fim ao Problema 2.3.3 proposto por Milnor. Para isso, usaremos ferramentas da geometria e topologia de espaços de configuração, conforme [19], e um resultado de L. Funar, apresentado na Seção 2.5 deste trabalho. Mais precisamente, primeiro classificaremos fibrados $E^{5} \rightarrow S^{2}$ que restritos ao bordo são triviais (sendo que a trivialização é fixada) ${ }^{1}$ e cuja fibra é a 3-esfera com a união disjunta dos interiores de 3-discos removida e em seguida, mostraremos que as classes de isomorfismo de tais fibrados estão em correspondência $1-1$ com o segundo grupo de homotopia de um certo espaço de configuração, cujos elementos correspondem a uma matriz de inteiros anti-simétrica. Então, mostraremos que um dado fibrado $E^{5} \rightarrow S^{2}$ está associado a um NS-par $\left(S^{5}, K\right)$ se, e somente se, a matriz antisimétrica é unimodular. Como uma consequência, veremos que o número de componentes de bordo de uma fibra é sempre ímpar, o que nos permitirá construir muitos NS-pares $\left(S^{5}, K\right)$ não-triviais, e então a construção de Looijenga, que foi apresentada na Seção 2.4, fornecerá germes de aplicações polinomiais não-triviais com uma singularidade isolada na origem.

Todos os principais resultados deste capítulo estão reunidos em forma de artigo em $[5]$.

\subsection{Classificação de fibrados}

Seja $\left(S^{5}, K^{2}\right)$ um NS-par, onde $K^{2}$ é uma variedade 2-dimensional mergulhada na esfera 5-dimensional $S^{5}$. Considere a fibração associada $\pi: S^{5} \backslash \operatorname{Int} N\left(K^{2}\right) \rightarrow S^{2}$, onde $N\left(K^{2}\right)$ denota uma vizinhança tubular fechada de $K^{2}$ em $S^{5}$, e denote por $F$ sua fibra, a qual é uma variedade 3-dimensional compacta cujo bordo é difeomorfo a $K^{2}$. De acordo

\footnotetext{
${ }^{1}$ Uma vez que a trivialização for fixada, não mais haverá mudança.
} 
com o Lema 2.3.5, $S^{5}$ não fibra sobre $S^{2}$, assim temos $K^{2} \neq \emptyset$. Além disso, temos a sequência exata em homotopia

$$
\pi_{2}\left(S^{5} \backslash \operatorname{Int} N\left(K^{2}\right)\right) \rightarrow \pi_{2}\left(S^{2}\right) \rightarrow \pi_{1}(F) \rightarrow \pi_{1}\left(S^{5} \backslash \operatorname{Int} N\left(K^{2}\right)\right) .
$$

Como $\pi$ é trivial sobre o bordo, ela tem uma seção, de modo que o homomorfismo $\pi_{2}\left(S^{5} \backslash\right.$ Int $\left.N\left(K^{2}\right)\right) \rightarrow \pi_{2}\left(S^{2}\right)$ é sobrejetor. Mais ainda, $S^{5} \backslash \operatorname{Int} N\left(K^{2}\right)$ é simplesmente conexa ${ }^{2}$. Portanto, a variedade 3-dimensional compacta $F$ é também simplesmente conexa.

Pelo Lema 3.0.6, vemos que $K^{2} \approx \partial F$ consiste de algumas cópias de $S^{2}$. Além disso, $F$ é equivalente homotópica a uma esfera 3-dimensional com alguns pontos removidos. Então, pelo fato da Conjectura de Poincaré ser verdadeira, temos que $F$ é difeomorfo à 3-esfera com os interiores de $k+1$ bolas disjuntas 3-dimensionais removidos, para algum inteiro não-negativo $k$. Dessa forma, denotaremos $F$ por $S_{(k+1)}^{3}=S^{3} \backslash \cup^{k+1} \operatorname{Int} B^{3}$. Nesta seção, classificamos tais fibrados.

É conhecido (ver, por exemplo, [23]) que

$$
\operatorname{Diff}\left(S^{n}\right) \simeq O(n+1) \times \operatorname{Diff}\left(B^{n}, \partial B^{n}\right),
$$

onde $\operatorname{Diff}\left(S^{n}\right)$ é o grupo de difeomorfismos da esfera $S^{n}, O(n+1)$ é o grupo ortogonal e $\operatorname{Diff}\left(B^{n}, \partial B^{n}\right)$ é o grupo de difeomorfismos da bola $B^{n}$, que restritos ao bordo $\partial B^{n}$ coincidem com a identidade.

Seja Diff $\left(S^{3}\right)$ o grupo topológico (munido da topologia $C^{\infty}$ ) de difeomorfismos de $S^{3}$. A Conjectura de Smale afirma que a inclusão de $O(4)$ em $\operatorname{Diff}\left(S^{3}\right)$, é uma equivalência de homotopia. Desta forma, por (4.1.1), temos que a Conjectura de Smale é equivalente a: $\operatorname{Diff}\left(B^{3}, \partial B^{3}\right) \simeq *$, ou seja, $\operatorname{Diff}\left(B^{3}, \partial B^{3}\right)$ é contrátil, onde $B^{3}$ é a bola 3 -dimensional fechada.

Como tal conjectura foi provada positivamente por Hatcher em [23], temos que $\operatorname{Diff}\left(S^{3}\right)$ é equivalente homotópico ao grupo ortogonal $O(4)$.

Vamos denotar por $\cup^{k+1} B^{3}$ a união disjunta de $k+1$ cópias de $B^{3}$, para um inteiro não-negativo $k$. Considere um mergulho padrão de $\cup^{k+1} B^{3}$ em $S^{3}$ (aplicação inclusão) e denote-o por $j_{k+1}$.

Denotamos por $\operatorname{Emb}\left(\cup^{k+1} B^{3}, S^{3}\right)$ o espaço de todos os mergulhos suaves de $\cup^{k+1} B^{3}$ em $S^{3}$, não necessariamente o padrão, e por $\operatorname{Diff}\left(S^{3}, \cup^{k+1} B^{3}\right)$ o subespaço de $\operatorname{Diff}\left(S^{3}\right)$ consistindo daqueles difeomorfismos que restritos à $\cup^{k+1} B^{3}$ coincidem com a aplicação inclusão $j_{k+1}$. Além disso, denotamos por $\operatorname{Diff}\left(S_{(k+1)}^{3}, \partial S_{(k+1)}^{3}\right)$ o grupo topológico de difeomorfismos de $S_{(k+1)}^{3}$ que se restringem à identidade no bordo.

O próximo lema segue de [11, Proposição 1, p. 120].

\footnotetext{
${ }^{2}$ Isto decorre da construção utilizada para recuperar a esfera na demonstração do Lema 2.2.4.
} 
Lema 4.1.1. A aplicação canônica $\operatorname{Diff}\left(S^{3}, \cup^{k+1} B^{3}\right) \rightarrow \operatorname{Diff}\left(S_{(k+1)}^{3}, \partial S_{(k+1)}^{3}\right)$ induz isomorfismos

$$
\pi_{i}\left(\operatorname{Diff}\left(S^{3}, \cup^{k+1} B^{3}\right)\right) \rightarrow \pi_{i}\left(\operatorname{Diff}\left(S_{(k+1)}^{3}, \partial S_{(k+1)}^{3}\right)\right)
$$

para todo $i$.

Agora considere a aplicação natural

$$
\gamma: \operatorname{Diff}\left(S^{3}\right) \rightarrow \operatorname{Emb}\left(\cup^{k+1} B^{3}, S^{3}\right)
$$

que envia cada difeomorfismo de $S^{3}$ para sua restrição a $\cup^{k+1} B^{3}$.

O resultado a seguir é uma consequência do teorema da fibração de Cerf-Palais (ver Observação 1.2.13, [11, Apêndice], [41]).

Lema 4.1.2. A aplicação natural $\gamma$ como acima é a projeção de um fibrado localmente trivial com fibra $\operatorname{Diff}\left(S^{3}, \cup^{k+1} B^{3}\right)$.

Portanto, pelo Teorema 1.2.11, temos a seguinte sequência exata em homotopia

$$
\begin{aligned}
& \pi_{2}\left(\operatorname{Diff}\left(S^{3}\right), \mathrm{id}\right) \rightarrow \pi_{2}\left(\operatorname{Emb}\left(\cup^{k+1} B^{3}, S^{3}\right), j_{k+1}\right) \rightarrow \pi_{1}\left(\operatorname{Diff}\left(S^{3}, \cup^{k+1} B^{3}\right), \mathrm{id}\right) \\
\rightarrow & \pi_{1}\left(\operatorname{Diff}\left(S^{3}\right), \mathrm{id}\right) \rightarrow \pi_{1}\left(\operatorname{Emb}\left(\cup^{k+1} B^{3}, S^{3}\right), j_{k+1}\right) \rightarrow \cdots .
\end{aligned}
$$

Seja $\mathbb{F}_{k+1}\left(S^{3}\right)$ o espaço de configuração de $k+1$ pontos em $S^{3}$. Esse espaço pode ser naturalmente identificado $\operatorname{com} \operatorname{Emb}\left(\{0,1, \ldots, k\}, S^{3}\right)$.

Lema 4.1.3. O espaço $\operatorname{Emb}\left(\cup^{k+1} B^{3}, S^{3}\right)$ é equivalente homotópico a $\mathbb{F}_{k+1}\left(S^{3}\right) \times O(3)^{k+1}$.

Demonstração. Para um dado mergulho $\eta: \cup^{k+1} B^{3} \rightarrow S^{3}$, associamos o elemento de $\mathbb{F}_{k+1}\left(S^{3}\right)$ que envia o $i$-ésimo ponto para a $\eta$-imagem do centro da $i$-ésima 3 -bola. Além disso, pela associação da diferencial normalizada de $\eta$ em cada centro, obtemos um elemento de $O(3)^{k+1}$. (Note que o fibrado tangente $T S^{3}$ de $S^{3}$ é trivial, e fixamos sua trivialização.) Então, podemos mostrar que a aplicação $\operatorname{Emb}\left(\cup^{k+1} B^{3}, S^{3}\right) \rightarrow \mathbb{F}_{k+1}\left(S^{3}\right) \times O(3)^{k+1}$ assim obtida é uma equivalência de homotopia. (Por exemplo, ver [11, Apêndice, §5, Proposição 3].)

Relembre que $\operatorname{Diff}\left(S^{3}\right) \simeq O(4)$ e como $O(3)$ e $O(4)$ são grupos de Lie compactos, temos pela Proposição 1.4.4 que $\pi_{2}(O(3))=0=\pi_{2}\left(O(4)\right.$ ). Além disso, $\mathbb{F}_{k+1}\left(S^{3}\right)$ é 1-conexo (ver [19]). Assim, a sequência exata (4.1.2) se transforma em

$$
0 \rightarrow \pi_{2}\left(\mathbb{F}_{k+1}\left(S^{3}\right)\right) \rightarrow \pi_{1}\left(\operatorname{Diff}\left(S^{3}, \cup^{k+1} B^{3}\right), \text { id }\right) \rightarrow \pi_{1}(O(4), \text { id }) \rightarrow \pi_{1}\left(O(3)^{k+1}, \text { id }\right) \rightarrow \cdots
$$


Note que $\pi_{1}(O(4)$, id $) \cong \mathbb{Z}_{2}$ e $\pi_{1}\left(O(3)^{k+1}\right.$, id $) \cong\left(\mathbb{Z}_{2}\right)^{k+1}$ (para maiores detalhes, ver [24, p.384]). Escolhendo o mergulho padrão $j_{k+1}$ de modo que seja equivariante com respeito às $S O(2)$-ações naturais, vemos que o homomorfismo $\pi_{1}(O(4)$, id $) \rightarrow \pi_{1}\left(O(3)^{k+1}\right.$, id) envia o gerador $1 \in \mathbb{Z}_{2}$ para $(1,1, \ldots, 1) \in\left(\mathbb{Z}_{2}\right)^{k+1}$. Em particular, ele é injetor. Assim, vemos que o homomorfismo

$$
\pi_{2}\left(\mathbb{F}_{k+1}\left(S^{3}\right)\right) \rightarrow \pi_{1}\left(\operatorname{Diff}\left(S^{3}, \cup^{k+1} B^{3}\right), \mathrm{id}\right)
$$

é um isomorfismo.

Por [19], e pelo Lema 2.5.2, temos o seguinte.

Lema 4.1.4. O grupo de homotopia $\pi_{2}\left(\mathbb{F}_{k+1}\left(S^{3}\right)\right)$ é isomorfo a $\mathbb{Z}^{k(k-1) / 2}$.

Note que, para um fibrado suave $S_{(k+1)}^{3} \hookrightarrow E^{5} \rightarrow S^{2}$ com grupo estrutural

$$
\operatorname{Diff}\left(S_{(k+1)}^{3}, \partial S_{(k+1)}^{3}\right)
$$

sua aplicação característica é um elemento de

$$
\pi_{1}\left(\operatorname{Diff}\left(S_{(k+1)}^{3}, \partial S_{(k+1)}^{3}\right), \mathrm{id}\right)
$$

o qual é isomorfo a $\pi_{1}\left(\operatorname{Diff}\left(S^{3}, \cup^{k+1} B^{3}\right)\right.$,id $) \cong \mathbb{Z}^{k(k-1) / 2}$ por Lema 4.1 .1 , isomorfismo (4.1.4) e Lema 4.1.4.

De fato, dado um tal fibrado suave $\pi: E^{5} \rightarrow S^{2}$, pode-se considerar $S^{2}=D_{1}^{2} \cup D_{2}^{2}$, onde $D_{i}^{2}, i=1,2$, denota o disco fechado 2 -dimensional. Como cada $D_{i}^{2}$ é contrátil, a restrição $\pi: \pi^{-1}\left(D_{i}^{2}\right) \rightarrow D_{i}^{2}$ é um fibrado trivial com fibra $S_{(k+1)}^{3}$, e temos $\pi^{-1}\left(D_{i}^{2}\right) \approx D_{i}^{2} \times S_{(k+1)}^{3}$. Assim, podemos recuperar o espaço total a partir da colagem

$$
E^{5}=\left(D_{1}^{2} \times S_{(k+1)}^{3}\right) \cup_{h}\left(D_{2}^{2} \times S_{(k+1)}^{3}\right)
$$

para algum difeomorfismo $h: \partial D_{2}^{2} \times S_{(k+1)}^{3} \rightarrow \partial D_{1}^{2} \times S_{(k+1)}^{3}$ definido por $h(t, x)=$ $(t, \alpha(t)(x))$, onde $\alpha: S^{1}=\partial D^{2} \rightarrow \operatorname{Diff}\left(S_{(k+1)}^{3}, \partial S_{(k+1)}^{3}\right)$ corresponde à aplicação característica. Portanto, a estrutura do fibrado fica completamente determinada pela classe de homotopia $[\alpha] \in \pi_{1}\left(\operatorname{Diff}\left(S_{(k+1)}^{3}, \partial S_{(k+1)}^{3}\right)\right.$,id $)$.

\subsection{Caracterização de NS-pares}

Para um inteiro não-negativo $k$, seja

$$
S_{(k+1)}^{3} \hookrightarrow E^{5} \stackrel{\pi}{\rightarrow} S^{2}
$$


um fibrado suave tal que sua restrição ao bordo $\partial S_{(k+1)}^{3} \hookrightarrow \partial E^{5} \rightarrow S^{2}$ é um fibrado trivial cuja trivialização é fixada. Note que o grupo estrutural de tal fibrado é considerado como sendo $\operatorname{Diff}\left(S_{(k+1)}^{3}, \partial S_{(k+1)}^{3}\right)$, que age sobre $S_{(k+1)}^{3}$ pela identidade sobre seu bordo, uma vez que a trivialização da fibração no bordo está fixada. Nesta seção, caracterizamos tais fibrados que surgem de um NS-par $\left(S^{5}, K\right)$ com $K \approx \cup^{k+1} S^{2}$.

Começamos colando o fibrado trivial $\cup^{k}\left(B^{3} \times S^{2}\right) \rightarrow S^{2}$ ao longo de $k$ componentes de bordo na fibração (4.2.1) a fim de obtermos a $B^{3}$-fibração

$$
\tilde{\pi}: Y=E^{5} \cup\left(\cup^{k}\left(B^{3} \times S^{2}\right)\right) \rightarrow S^{2} .
$$

Note que ao colarmos o fibrado trivial $\cup^{k}\left(B^{3} \times S^{2}\right) \rightarrow S^{2}$ na fibração (4.2.1) ao longo da fibração no bordo, usamos a identificação natural de $\partial B^{3}$ e cada componente de $\partial S_{(k+1)}^{3}$ junto com a trivialização fixada da fibração no bordo. A fibração resultante (4.2.2) é trivial, pois, como vimos na Seção 4.1, o grupo estrutural Diff $\left(B^{3}, \partial B^{3}\right)$ é contrátil. Portanto, o espaço total $Y$ da fibração (4.2.2) é difeomorfo a $B^{3} \times S^{2}$. Por outro lado, note que

$$
\begin{aligned}
S^{5} & =\partial B^{6}=\partial\left(B^{3} \times B^{3}\right) \\
& =\left(\partial B^{3} \times B^{3}\right) \cup\left(B^{3} \times \partial B^{3}\right) \\
& =\left(S^{2} \times B^{3}\right) \cup\left(B^{3} \times S^{2}\right)
\end{aligned}
$$

e sendo $N\left(S^{2}\right)=S^{2} \times B^{3}$, concluímos que

$$
S^{5} \backslash N\left(S^{2}\right)=B^{3} \times S^{2} \approx Y
$$

Então, colando $B_{0}^{3} \times S^{2}$ a $Y=B^{3} \times S^{2}$ pela aplicação $\partial B_{0}^{3} \times S^{2} \rightarrow \partial B^{3} \times S^{2}$ dada por $(x, y) \mapsto(y, x)$, obtemos a esfera $S^{5}$, onde $B_{0}^{3}$ é uma cópia da bola 3-dimensional fechada. Considere $S_{0}^{2}=x_{0} \times S^{2}$, onde $x_{0}$ é o centro de $B_{0}^{3}$, e escreva $N\left(S_{0}^{2}\right)=B_{0}^{3} \times S^{2}$, o qual é identificado com a vizinhança tubular fechada de $S_{0}^{2}$ em $S^{5}$. Dessa forma, podemos identificar o espaço $Y$ com o complemento de uma vizinhança tubular aberta de $S_{0}^{2}$ em $S^{5}$, isto é,

$$
Y=S^{5} \backslash \operatorname{Int} N\left(S_{0}^{2}\right)
$$

Para fixar a notação escrevemos $\cup^{k+1} B^{3}=\cup_{i=0}^{k} B_{i}^{3}$, e denotamos por $x_{i}$ o centro de $B_{i}^{3}$, onde consideramos $\cup^{k} B^{3}=\cup_{i=1}^{k} B_{i}^{3}$. Também escrevemos $S_{i}^{2}=x_{i} \times S^{2}, i=1,2, \ldots, k$. Note que as esferas 2-dimensionais $S_{i}^{2}, i=0,1, \ldots, k$, estão mergulhadas em $S^{5}$ de uma forma padrão. Além disso, cada $S_{i}^{2}, i=1,2, \ldots, k$, tem linking number $\pm 1 \operatorname{com} S_{0}^{2}$. Nas discussões a seguir, orientamos $S_{i}^{2}, i=0,1,2 \ldots, k$, de tal forma que o linking number de $S_{i}^{2} \operatorname{com} S_{0}^{2}$ é igual a $+1, i=1,2, \ldots, k$. 

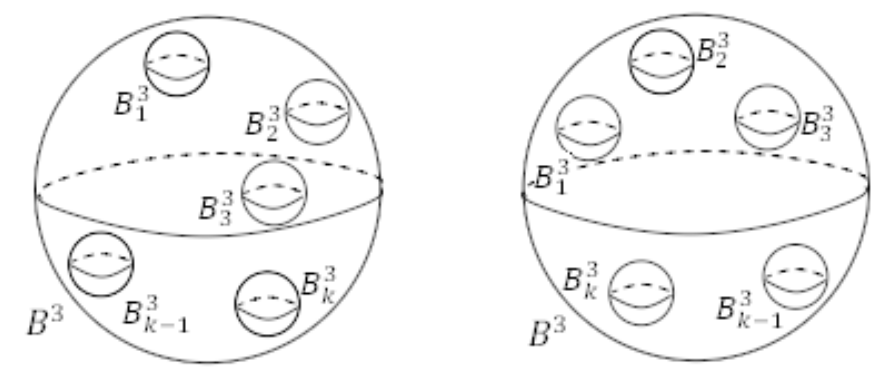

Figura 4.1: Representação de duas fibras distintas da $B^{3}$-fibração $\widetilde{\pi}$

Para $y \in S^{2}$, podemos representar a fibra $\tilde{\pi}^{-1}(y)$ por $B^{3} \times\{y\}$. Assim, é fácil ver que

$$
\tilde{\pi}^{-1}(y) \cap\left(\cup_{i=1}^{k} B_{i}^{3} \times S^{2}\right)=\cup_{i=1}^{k}\left(B_{i}^{3} \times y\right) .
$$

Desta forma, temos $\cup_{i=1}^{k}\left(B_{i}^{3} \times y\right) \subset \widetilde{\pi}^{-1}(y) \approx B^{3}$. (Veja na Figura 4.1, a representação das fibras $\widetilde{\pi}^{-1}\left(y_{1}\right)$ e $\widetilde{\pi}^{-1}\left(y_{2}\right)$, para $y_{1}, y_{2} \in S^{2}$ e $y_{1} \neq y_{2}$.) Portanto, para cada $y \in S^{2}$ podemos associar naturalmente um elemento do espaço de configuração $\mathbb{F}_{k}\left(\operatorname{Int} B^{3}\right) \approx \mathbb{F}_{k}\left(\mathbb{R}^{3}\right)$. Isso define uma aplicação classificante $c: S^{2} \rightarrow \mathbb{F}_{k}\left(\mathbb{R}^{3}\right)$.

Então, temos o seguinte.

Lema 4.2.1. As classes de isomorfismo das fibrações como em (4.2.1) estão em correspondência $1-1 \operatorname{com} \pi_{2}\left(\mathbb{F}_{k}\left(\mathbb{R}^{3}\right)\right) \cong \mathbb{Z}^{k(k-1) / 2}$. A correspondência é dada pela associação com a classe de homotopia da aplicação classificante $c$.

Demonstração. De acordo com o que vimos na Seção 4.1, as classes de isomorfismo dos fibrados em questão estão em correspondência 1 - 1 com

$$
\pi_{2}\left(\mathbb{F}_{k+1}\left(S^{3}\right)\right) \cong \pi_{1}\left(\operatorname{Diff}\left(S^{3}, \cup^{k+1} B^{3}\right), \mathrm{id}\right) .
$$

Por outro lado, é conhecido que $\pi_{2}\left(\mathbb{F}_{k}\left(\mathbb{R}^{3}\right)\right)$ é naturalmente isomorfo a $\pi_{2}\left(\mathbb{F}_{k+1}\left(S^{3}\right)\right)$ (ver [19, p. 38]).

Relembre o fibrado localmente trivial

$$
\operatorname{Diff}\left(S^{3}, \cup^{k+1} B^{3}\right) \stackrel{\iota}{\longrightarrow} \operatorname{Diff}\left(S^{3}\right) \stackrel{\varphi}{\longrightarrow} \operatorname{Emb}\left(\cup^{k+1} B^{3}, S^{3}\right)
$$

do Lema 4.1.2, onde $\iota$ é a inclusão natural. Para a classe de homotopia

$$
[\alpha] \in \pi_{1}\left(\operatorname{Diff}\left(S_{(k+1)}^{3}, \partial S_{(k+1)}^{3}\right), \operatorname{id}\right) \cong \pi_{1}\left(\operatorname{Diff}\left(S^{3}, \cup^{k+1} B^{3}\right), \mathrm{id}\right)
$$

da aplicação característica, sua imagem por $\iota_{*}$ se anula em $\pi_{1}\left(\operatorname{Diff}\left(S^{3}\right)\right)$, de modo que existe $^{3}$ uma aplicação contínua $\tilde{\alpha}: D^{2} \rightarrow \operatorname{Diff}\left(S^{3}\right)$ que estende $\iota \circ \alpha: S^{1} \rightarrow \operatorname{Diff}\left(S^{3}\right)$. Então, a classe de homotopia de $\varphi \circ \tilde{\alpha}: D^{2} \rightarrow \operatorname{Emb}\left(\cup^{k+1} B^{3}, S^{3}\right)$ em $\pi_{2}\left(\operatorname{Emb}\left(\cup^{k+1} B^{3}, S^{3}\right)\right) \cong$

\footnotetext{
${ }^{3}$ Confira o Teorema 1.1.11.
} 
$\pi_{2}\left(\mathbb{F}_{k+1}\left(S^{3}\right)\right) \cong \pi_{2}\left(\mathbb{F}_{k}\left(\mathbb{R}^{3}\right)\right)$ é a classe correspondente a $[\alpha]$ pelo isomorfismo (4.1.4). Por construção, isso coincide com a classe de homotopia da aplicação classificante $c$.

Agora, temos a seguinte questão natural.

Problema 4.2.2. Quais elementos de $\pi_{2}\left(\mathbb{F}_{k}\left(\mathbb{R}^{3}\right)\right) \cong \mathbb{Z}^{k(k-1) / 2}$ correspondem a um NS-par?

Respondemos esta questão em nosso principal resultado deste capítulo, como segue.

Teorema 4.2.3. O fibrado $S_{(k+1)}^{3} \hookrightarrow E^{5} \stackrel{\pi}{\rightarrow} S^{2}$ como em (4.2.1) surge de um NS-par se, e somente se, $\operatorname{det}\left(\operatorname{lk}\left(S_{i}^{2}, S_{j}^{2}\right)\right)_{1 \leqslant i, j \leqslant k}=1$, onde $\operatorname{lk}\left(S_{i}^{2}, S_{i}^{2}\right)=0$ para todo $1 \leqslant i \leqslant k$, por convenção.

Relembre que, de acordo com o Lema 2.5.2, um fibrado (4.2.1) corresponde a um elemento de $\mathbb{Z}^{k(k-1) / 2}$, o qual é representado pela matriz $k \times k$ anti-simétrica e cujas entradas são inteiros, $\left(\operatorname{lk}\left(S_{i}^{2}, S_{j}^{2}\right)\right)_{1 \leqslant i<j \leqslant k}$, onde lk denota o linking number em $S^{5}$ e as orientações de $S^{5}$ e de $S_{i}^{2}, i=1,2, \ldots, k$ são fixadas.

O resto da seção é dedicado à prova do teorema acima.

Considere o fibrado como em (4.2.1). Fixamos a trivialização da fibração no bordo, e escrevemos $\partial E^{5}=\cup_{i=0}^{k}\left(K_{i} \times S^{2}\right)$, onde $K_{i} \approx S^{2}$ são as componentes de bordo da fibra $S_{(k+1)}^{3}$ e são orientadas de tal forma que o ciclo representado por $K_{0}$ é homólogo à soma dos ciclos representados por $K_{i}, i=1,2, \ldots, k$. Seja $X^{5}=E^{5} \cup\left(\cup_{i=0}^{k}\left(K_{i} \times B^{3}\right)\right)$ a variedade fechada 5-dimensional obtida da colagem de $E^{5}$ e $\cup_{i=0}^{k}\left(K_{i} \times B^{3}\right)$ ao longo de seus bordos, de tal forma que a projeção natural

$$
\cup_{i=0}^{k}\left(K_{i} \times\left(B^{3} \backslash\{0\}\right)\right) \rightarrow S^{2}
$$

se estende para uma fibração suave $X^{5} \backslash K \rightarrow S^{2}$, onde $K=\cup_{i=0}^{k}\left(K_{i} \times\{0\}\right)$. Note que, nesta notação, $K_{i}$ é identificado com $\partial B_{i}^{3}, i=1,2, \ldots, k$, e $K_{0}$ é identificado com $* \times S^{2} \subset \partial B_{0}^{3} \times S^{2}$. É importante observar que a maneira como $K_{i} \times B^{3}$ são atachadas a $E^{5}$ é muito diferente daquela feita na construção de $Y \subset S^{5}$ em (4.2.2).

Observações 4.2.4. (a) A fibra $S_{(k+1)}^{3}$ tem o tipo de homotopia de um wedge de 2-esferas $\bigvee_{i=1}^{k} S_{i}^{2}$, consequentemente

$$
H_{q}\left(S_{(k+1)}^{3}\right)=\left\{\begin{array}{l}
\mathbb{Z}, \text { se } q=0 \\
\mathbb{Z}^{k}, \text { se } q=2 \\
0, \text { caso contrário. }
\end{array}\right.
$$


(b) Sabendo que $E^{5} \cap\left(\cup_{i=0}^{k}\left(K_{i} \times B^{3}\right)\right)=\partial E^{5}=\cup_{i=0}^{k}\left(K_{i} \times \partial B^{3}\right)=\bigsqcup^{k+1} S^{2} \times S^{2}$, temos

$$
H_{q}\left(\cup_{i=0}^{k}\left(K_{i} \times \partial B^{3}\right)\right)=\left\{\begin{array}{l}
\mathbb{Z}^{k+1}, \text { se } q=0,4 \\
\mathbb{Z}^{2(k+1)}, \text { se } q=2, \\
0, \text { caso contrário. }
\end{array}\right.
$$

(c) Aplicando a sequência exata de Wang (ver Proposição 1.2.12) para a fibração $F \hookrightarrow$ $E^{5} \rightarrow S^{2}$ obtemos (iniciando em $q=4$ )

$$
\begin{aligned}
\cdots & \rightarrow H_{4}(F) \rightarrow H_{4}\left(E^{5}\right) \rightarrow H_{2}(F) \\
& \rightarrow H_{3}(F) \rightarrow H_{3}\left(E^{5}\right) \rightarrow H_{1}(F) \\
& \rightarrow H_{2}(F) \rightarrow H_{2}\left(E^{5}\right) \rightarrow H_{0}(F) \\
& \rightarrow H_{1}(F) \rightarrow H_{1}\left(E^{5}\right) \rightarrow 0 \\
& \rightarrow H_{0}(F) \rightarrow H_{0}\left(E^{5}\right) \rightarrow 0 .
\end{aligned}
$$

Consequentemente,

$$
H_{q}\left(E^{5}\right)=\left\{\begin{array}{l}
\mathbb{Z}, \text { se } q=0 \\
\mathbb{Z}^{k+1}, \text { se } q=2 \\
\mathbb{Z}^{k}, \text { se } q=4 \\
0, \text { caso contrário. }
\end{array}\right.
$$

O Teorema 4.2.3 é uma consequência dos Lemas 4.2.5 e 4.2.6 abaixo.

Observe que, por construção, se $X^{5}$ é difeomorfa à $S^{5}$, temos um NS-par $\left(X^{5}, K\right)$ por definição. Além disso, como uma consequência da Proposição 1.2.24, juntamente com o item (1) das Observações 1.2.25, podemos enunciar o resultado a seguir.

Lema 4.2.5. O fibrado $\pi$ como em (4.2.1) surge de um NS-par se, e somente se, $X^{5}$ é equivalente homotópico a $S^{5}$.

Pelas observações supracitadas e pelo fato de $X^{5}$ ser simplesmente conexo, é suficiente estudar o grupo de homologia $H_{2}\left(X^{5}\right)$. Então considere o seguinte pedaço da sequência exata de Mayer-Vietoris:

$$
H_{2}\left(\cup_{i=0}^{k}\left(K_{i} \times \partial B^{3}\right)\right) \stackrel{\rho}{\rightarrow} H_{2}\left(E^{5}\right) \oplus H_{2}\left(\cup_{i=0}^{k}\left(K_{i} \times B^{3}\right)\right) \rightarrow H_{2}\left(X^{5}\right) \rightarrow 0,
$$

onde o homomorfismo $\rho=\left(i_{1 *},-i_{2 *}\right)$ é induzido pelas inclusões $i_{1}: \cup_{i=0}^{k}\left(K_{i} \times \partial B^{3}\right) \rightarrow E^{5}$ e $i_{2}: \cup_{i=0}^{k}\left(K_{i} \times \partial B^{3}\right) \rightarrow \cup_{i=0}^{k}\left(K_{i} \times B^{3}\right)$.

A Figura a seguir nos ajuda a entender as imagens dos elementos de $H_{2}\left(\cup_{i=0}^{k}\left(K_{i} \times\right.\right.$ $\left.\partial B^{3}\right)$ ) pelo homomorfismo $\rho$. Contudo, ela retrata a situação em $S^{5}$ e não em $X^{5}$. 


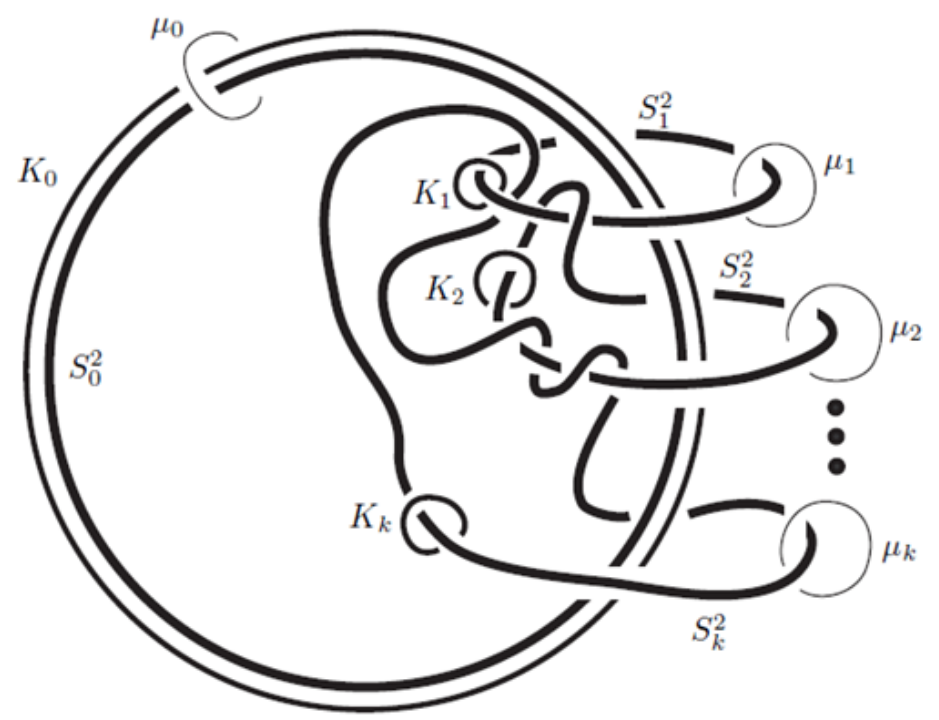

A fim de descrever o homomorfismo $\rho$, vamos fixar bases dos grupos de homologia. Na sequência, para um ciclo $c$, denotamos por $[c]$ a classe de homologia representada por $c$. Primeiro, temos

$$
H_{2}\left(\cup_{i=0}^{k}\left(K_{i} \times \partial B^{3}\right)\right) \cong \oplus_{i=0}^{k} H_{2}\left(K_{i} \times \partial B^{3}\right)
$$

e cada $H_{2}\left(K_{i} \times \partial B^{3}\right) \cong \mathbb{Z} \oplus \mathbb{Z}$ é gerado por $\left[K_{i} \times *\right]$ e $\left[y_{i} \times \partial B^{3}\right]$, onde $y_{i} \in K_{i}, i=0,1, \ldots, k$. Além disso, temos

$$
H_{2}\left(\cup_{i=0}^{k}\left(K_{i} \times B^{3}\right)\right) \cong \oplus_{i=0}^{k} H_{2}\left(K_{i} \times B^{3}\right)
$$

e cada $H_{2}\left(K_{i} \times B^{3}\right) \cong \mathbb{Z}$ é gerado por $\delta_{i}=\left[K_{i} \times *\right], * \in B^{3}, i=0,1, \ldots, k$. Por outro lado, usando as identificações para $Y$ apresentadas em (4.2.2) e (4.2.3), temos

$$
E^{5}=S^{5} \backslash\left(\cup_{i=0}^{k} \operatorname{Int} N\left(S_{i}^{2}\right)\right),
$$

onde $N\left(S_{i}^{2}\right)=B_{i}^{3} \times S^{2}$ é a vizinhança tubular fechada de $S_{i}^{2}$ em $S^{5}$, e $S_{i}^{2}=x_{i} \times S^{2}$, $i=1,2, \ldots, k$, são os duais de Hopf de $S_{0}^{2}$. Portanto, pela dualidade de Alexander temos

$$
H_{2}\left(E^{5}\right) \cong H^{2}\left(\cup_{i=0}^{k} \operatorname{Int} N\left(S_{i}^{2}\right)\right) .
$$

Uma vez que $N\left(S_{i}^{2}\right)=B_{i}^{3} \times S^{2}$, podemos tomar os geradores $\mu_{i}=\left[\partial B_{i}^{3} \times *\right] \in H_{2}\left(E^{5}\right)$, $* \in S^{2}$, e $H_{2}\left(E^{5}\right)$ é gerado livremente por $\mu_{i}, i=0,1, \ldots, k$. Aqui, orientamos $\mu_{i}$ de tal forma que o linking number de $\mu_{i}$ com $S_{i}^{2}$ é igual a +1 . Observe que $\mu_{i}=i_{1 *}\left(\left[K_{i} \times *\right]\right)$ para $i=1,2, \ldots, k$, e $\delta_{i}=i_{2 *}\left(\left[K_{i} \times *\right]\right)$ para $i=0,1, \ldots, k$. Portanto, as imagens dos 
geradores pelo homomorfismo $\rho$ podem ser escritas como segue.

$$
\begin{aligned}
\rho\left(\left[K_{0} \times\{*\}\right]\right) & =\mu_{1}+\mu_{2}+\cdots+\mu_{k}-\delta_{0}, \\
\rho\left(\left[K_{i} \times\{*\}\right]\right) & =\mu_{i}-\delta_{i} \quad(1 \leqslant i \leqslant k), \\
\rho\left(\left[y_{0} \times \partial B^{3}\right]\right) & =\mu_{0}+0, \\
\rho\left(\left[y_{j} \times \partial B^{3}\right]\right) & =\sum_{0 \leqslant i \leqslant k, i \neq j} \operatorname{lk}\left(S_{j}^{2}, S_{i}^{2}\right) \mu_{i}+0 \quad(1 \leqslant j \leqslant k) .
\end{aligned}
$$

Assim, com respeito às bases acima, o homomorfismo $\rho$ é representado pela seguinte matriz:

$$
R=\left(\begin{array}{cccccccc}
0 & 0 & \cdots & 0 & 1 & 1 & \cdots & 1 \\
1 & 1 & \cdots & 0 & 0 & a_{11} & \cdots & a_{1 k} \\
\vdots & \vdots & \ddots & \vdots & \vdots & \vdots & \ddots & \vdots \\
1 & 0 & \cdots & 1 & 0 & a_{k 1} & \cdots & a_{k k} \\
& & & & & & & \\
-1 & 0 & \cdots & 0 & 0 & 0 & \cdots & 0 \\
0 & -1 & \cdots & 0 & 0 & 0 & \cdots & 0 \\
\vdots & \vdots & \ddots & \vdots & \vdots & \vdots & \ddots & \vdots \\
0 & 0 & \cdots & -1 & 0 & 0 & \cdots & 0
\end{array}\right)
$$

onde $a_{i j}=\operatorname{lk}\left(S_{j}^{2}, S_{i}^{2}\right), i \neq j, 1 \leqslant i, j \leqslant k$ e $a_{i i}=0$. Observe que $a_{i j}=-a_{j i}$.

Lema 4.2.6. A variedade 5-dimensional $X^{5}$ é equivalente homotópica a $S^{5}$ se, e somente se, $\operatorname{det} R= \pm 1$.

Demonstração. Se $X^{5}$ é equivalente homotópica a $S^{5}$, então seus segundo e terceiro grupos de homologia devem se anular e portanto o homomorfismo $\rho$ deve ser um isomorfismo, o qual implica que det $R \neq 0$. Como $R$ é uma matriz de inteiros cuja inversa também o é, temos que $\mathrm{R}$ é unimodular, ou seja, $\operatorname{det} R= \pm 1$.

Reciprocamente, se $\rho$ é um isomorfismo, pela sequência (4.2.4), temos $H_{2}\left(X^{5}\right)=0$. Uma vez que $X^{5}$ é simplesmente conexa, $H_{1}\left(X^{5}\right)=0$. Pela dualidade de Poincaré, temos

$$
\begin{aligned}
& H_{3}\left(X^{5}\right) \cong H^{2}\left(X^{5}\right) \cong \operatorname{Hom}\left(H_{2}\left(X^{5}\right), \mathbb{Z}\right) \cong 0 \\
& H_{4}\left(X^{5}\right) \cong H^{1}\left(X^{5}\right) \cong \operatorname{Hom}\left(H_{1}\left(X^{5}\right), \mathbb{Z}\right) \cong 0 \\
& H_{5}\left(X^{5}\right) \cong H^{0}\left(X^{5}\right) \cong \operatorname{Hom}\left(H_{0}\left(X^{5}\right), \mathbb{Z}\right) \cong \mathbb{Z}
\end{aligned}
$$

Assim, vemos que $X^{5}$ tem a homologia da $S^{5}$. Então, pelo Teorema de Whitehead (ver Teorema 1.1.9), $X^{5}$ é equivalente homotópica a $S^{5}$.

Isso completa a prova do Lema 4.2.6 e, assim, o Teorema 4.2.3 está provado. 
Corolário 4.2.7. Se o fibrado $S_{(k+1)}^{3} \hookrightarrow E^{5} \rightarrow S^{2}$ como em (4.2.1) surge de um NS-par, então $k$ deve ser par.

Demonstração. Uma matriz anti-simétrica de ordem $k$ tem determinante não nulo apenas se $k$ é par.

A seguinte proposição segue da construção de Looijenga, vista na Seção 2.4, e do resultado anterior.

Proposição 4.2.8. Para todo inteiro par $k \geqslant 0$, existe um $N S$-par $\left(S^{5}, L_{k+1}\right)$ com $L_{k+1}$ sendo difeomorfo à união disjunta de $k+1$ cópias de $S^{2}$, e existe um germe de aplicação polinomial $f:\left(\mathbb{R}^{6}, 0\right) \rightarrow\left(\mathbb{R}^{3}, 0\right)$ com um ponto crítico isolado na origem tal que o $N S$ par associado é isomorfo a $\left(S^{5}, L_{k+1} \sharp\left(-L_{k+1}\right)\right)$. Em particular, $L_{k+1} \sharp\left(-L_{k+1}\right)$ consiste de $2 k+1$ componentes conexas.

Demonstração. Primeiro, note que para cada inteiro positivo par $k$, existe uma matriz de inteiros anti-simétrica de determinante 1. (Por exemplo, considere a soma direta da matriz

$$
\left(\begin{array}{cc}
0 & 1 \\
-1 & 0
\end{array}\right)
$$

e suas cópias.) Agora lembre que o elemento $\left(\operatorname{lk}\left(S_{i}^{2}, S_{j}^{2}\right)\right)_{1 \leqslant i, j \leqslant k}$ corresponde a um fibrado como em (4.2.1). Então, do principal Teorema 4.2.3, existe um NS-par $\left(S^{5}, L_{k+1}\right)$ associado a tal fibrado e, consequentemente correspondente à tal matriz. Desta forma, pode-se aplicar a construção de Looijenga e o resultado segue.

Após todos esses resultados, estamos prontos para responder a questão do Milnor sobre não-trivialidade (Problema 2.3.3) para o par de dimensões $(6,3)$ como segue.

Corolário 4.2.9. Dado um germe de aplicação polinomial como na Proposição 4.2.8 com $k>0$, a fibra da fibração de Milnor associada não é difeomorfa a um disco.

\subsection{Uma generalização para dimensões altas}

Podemos generalizar a construção da Seção 4.2 em dimensões altas como segue, a fim de obtermos novos exemplos não-triviais de germes de aplicações polinomiais reais com uma singularidade isolada.

Seja $n \geqslant 3$ um inteiro. Para um inteiro não-negativo $k$, seja $S_{(k+1)}^{n}$ a esfera $n$ dimensional $S^{n}$ com o interior da união disjunta de $k+1$ cópias de discos $n$-dimensionais 
removido. Nesta seção, construiremos um fibrado suave

$$
S_{(k+1)}^{n} \hookrightarrow E^{2 n-1} \stackrel{\pi}{\rightarrow} S^{n-1}
$$

tal que a restrição ao bordo

$$
\partial S_{(k+1)}^{n} \hookrightarrow \partial E^{2 n-1} \stackrel{\pi}{\rightarrow} S^{n-1}
$$

é um fibrado trivial cuja trivialização está fixada, e tal que ele surge de um NS-par $\left(S^{2 n-1}, K^{n-1}\right)$.

Considere $A=\left(a_{i j}\right)$ uma matriz de inteiros $(-1)^{n}$-simétrica de ordem $k \times k$ tal que as entradas da diagonal são todas nulas. Seja $S_{0} \approx S^{n-1}$ uma $(n-1)$-esfera orientada, trivialmente mergulhada em $S^{2 n-1}$. Então, existem $(n-1)$-esferas orientadas $S_{i}$ suavemente mergulhadas em $S^{2 n-1}, i=1,2, \ldots, k$, tal que

(1) $S_{i}$ não intersecta $S_{0}$

(2) $S_{i}$ tem linking number +1 com $S_{0}$,

(3) o linking number $\operatorname{lk}\left(S_{i}, S_{j}\right)=a_{i j}, i \neq j$.

Tais mergulhos existem (por exemplo, veja [21]). Note, então, que

$$
E^{2 n-1}=S^{2 n-1} \backslash \cup_{i=0}^{k} \operatorname{Int} N\left(S_{i}\right)
$$

fibra naturalmente sobre $S^{n-1}$ de tal forma que a restrição ao bordo é trivial. (Mais precisamente, considere a sub-fibração associada do fibrado trivial $S^{2 n-1} \backslash \operatorname{Int} N\left(S_{0}\right) \approx$ $B^{n} \times S^{n-1} \rightarrow S^{n-1}$.) Então, pela mesma construção da Seção 4.2, obtemos um objeto $\left(X^{2 n-1}, K^{n-1}\right)$, onde $X^{2 n-1}$ é uma variedade suave fechada $(2 n-1)$-dimensional, $X^{2 n-1} \backslash$ Int $N\left(K^{n-1}\right)$ é difeomorfo a $E^{2 n-1}$, e ele fibra sobre $S^{n-1}$ com fibra $S_{(k+1)}^{n}$ de tal maneira que a aplicação projeção é compatível com a trivialização da vizinhança tubular fechada $N\left(K^{n-1}\right)$. Então temos o seguinte.

Lema 4.3.1. A variedade $X^{2 n-1}$ é uma $(2 n-1)$-esfera de homotopia se, e somente se, $\operatorname{det} A= \pm 1$.

Demonstração. Uma vez que $S_{(k+1)}^{n}$ tem o tipo de homotopia de um buquê de $k$ esferas $(n-1)$-dimensional, é fácil ver que $E^{2 n-1}$, e portanto $X^{2 n-1}$, é $(n-2)$-conexa. Desta forma, $H_{i}\left(X^{2 n-1}\right)=0, i=1, \ldots, n-2$. Pela dualidade de Poincaré, temos que $H_{j}\left(X^{2 n-1}\right)=$ $0, j=n+1, \ldots, 2 n-2$. Assim, $X^{2 n-1}$ é uma $(2 n-1)$-esfera de homotopia se, e somente se, $H_{n-1}\left(X^{2 n-1}\right)$ se anula. Então, um argumento usando uma sequência exata de MayerVietoris, como na seção anterior, conduz ao resultado desejado. 
Combinando isso com a construção de Looijenga, temos o seguinte.

Corolário 4.3.2. Seja $n \geqslant 3$ um inteiro. Para todo inteiro positivo $k$ com $k \equiv 1(\bmod 4)$, existe um NS-par $\left(S^{2 n-1}, L_{k}\right)$ com $L_{k}$ sendo difeomorfo à união disjunta de $k$ cópias de $S^{n-1}$, e existe um germe de aplicação polinomial $f:\left(\mathbb{R}^{2 n}, 0\right) \rightarrow\left(\mathbb{R}^{n}, 0\right)$ com uma singularidade isolada em 0 tal que o NS-par associado é isomorfo a $\left(S^{2 n-1}, L_{k} \sharp\left(-L_{k}\right)\right)$. Em particular, $L_{k} \sharp\left(-L_{k}\right)$ consiste de $2 k-1$ componentes conexas.

Note que a fibra de Milnor associada é difeomorfa à $S_{(2 k-1)}^{n}$ e é equivalente homotópica ao buquê de $2 k-2$ cópias da $(n-1)$-esfera.

Demonstração do Corolário 4.3.2. Seja $\ell=(k-1) / 2$, o qual é um inteiro par nãonegativo. Existe uma matriz $A$ de inteiros, $(-1)^{n}$-simétrica de ordem $\ell \times \ell$ com determinante \pm 1 . Por Haefliger [21], existe um mergulho $\cup_{i=0}^{k} S_{i}^{n-1}$ em $S^{2 n-1}$ tal que cada componente está trivialmente mergulhada, $S_{i}^{n-1}$ linca uma vez com $S_{0}$ para todo $i>0$, e a matriz $\left(l k\left(S_{i}^{n-1}, S_{j}^{n-1}\right)\right)_{1 \leqslant i, j \leqslant \ell}$ coincide com $A$, onde cada $S_{i}^{n-1}$ é uma cópia de $S^{n-1}$ e o linking number $\operatorname{lk}\left(S_{i}^{n-1}, S_{i}^{n-1}\right)=0, i=1,2, \ldots, \ell$, por convenção. Em seguida, pela construção acima, obtemos a esfera de homotopia $X^{2 n-1}$, na qual a união disjunta de $\ell+1$ cópias da $(n-1)$-esfera está mergulhada. Sendo $n \geqslant 3$, segue dos Lemas 1.2.26 e 1.2.27 e do Teorema 1.2.22 que a soma conexa $X^{2 n-1} \sharp\left(-X^{2 n-1}\right)$ é difeomorfa à $S^{2 n-1}$. Portanto, pela construção da soma conexa, obtemos um NS-par $\left(S^{2 n-1}, L_{k}\right)$, onde $L_{k}$ é difeomorfo à união disjunta de $2 \ell+1=k$ cópias de $S^{n-1}$. Então, aplicando a construção de Looijenga, obtemos a conclusão desejada. 


\section{Novos resultados sobre a topologia da fibra de Milnor real}

É conhecido que para um germe de função holomorfa $f:\left(\mathbb{C}^{n+1}, 0\right) \rightarrow(\mathbb{C}, 0)$ com uma singularidade isolada na origem, a fibra de Milnor $F_{f}$ tem o tipo de homotopia de um buquê (ou wedge) de esferas $n$-dimensionais. Por completitude, apresentaremos a prova de tal resultado na Seção 5.2. Para germes de aplicações polinomiais reais com singularidade isolada, não podemos esperar, em geral, um tal teorema do buquê, como pode ser visto na seção a seguir.

Contudo, apresentamos neste capítulo (ver também [5, Seção 5]), condições suficientes para garantirmos que uma fibra de Milnor real é equivalente homotópica a um buquê com um número não-nulo de esferas.

\subsection{Spinning para nó fibrado}

Pela construção do "twist spinning" na Seção 2.4 (ou veja [51] para mais detalhes), pode-se construir um NS-par $\left(S^{4}, K^{2}\right)$ tal que o grupo fundamental da fibra não é um grupo livre. Então a construção de Looijenga conduz a um germe de aplicação polinomial não-trivial $\left(\mathbb{R}^{5}, 0\right) \rightarrow\left(\mathbb{R}^{2}, 0\right)$ com uma singularidade isolada na origem, tal que a fibra de Milnor não tem grupo fundamental livre. Consequentemente, a fibra de Milnor não tem o tipo de homotopia de um buquê de esferas. Agora, aplicando uma vez a construção "spinning" explicada na Seção 2.4 para $\left(S^{4}, K^{2}\right)$, obtém-se um exemplo não-trivial em dimensões $(6,2)$ tal que a fibra de Milnor não é equivalente homotópica a um buquê de esferas. Realizando tais procedimentos indutivamente, pode-se construir exemplos em todos os pares de dimensões $(n, 2), n \geqslant 5$, tal que a fibra de Milnor não tem o tipo de homotopia de um buquê de esferas.

Na sequência, a fim de obtermos exemplos em altas dimensões, usamos a construção "spinning" devida a Artin [7]. Para um melhor entendimento, vamos relembrar a 
construção. Sejam $\left(S^{m}, K^{k}\right)$ um NS-par com $K^{k} \neq \emptyset$ e $\pi: S^{m} \backslash K^{k} \rightarrow S^{m-k-1}$ a fibração associada. Denotamos a fibra da fibração $S^{m} \backslash \operatorname{Int} N\left(K^{k}\right) \rightarrow S^{m-k-1}$ por $F^{k+1}$, onde $N\left(K^{k}\right)$ é a vizinhança tubular fechada de $K^{k}$ em $S^{m}$. Consideramos um ponto $q \in K^{k}$ e uma pequena vizinhança $m$-disco $D$ em $S^{m}$ tal que $\left(D, D \cap K^{k}\right)$ é difeomorfo ao par de discos padrão $\left(D^{m}, D^{k}\right)$ e tal que $\pi$ restrito a $D \backslash\left(D \cap K^{k}\right)$ é equivalente à fibração padrão $D^{m} \backslash D^{k} \rightarrow S^{m-k-1}$. Então, consideramos o espaço quociente de $\left(S^{m} \backslash \operatorname{Int} D, K^{k} \backslash\left(\operatorname{Int} D \cap K^{k}\right)\right) \times S^{1}$, onde para cada $x \in \partial D$, os pontos da forma $(x, t)$ são identificados a um ponto para todo $t$. Esse tipo de construção é chamado spinning. Como resultado, obtemos o par $\left(S^{m+1}, \tilde{K}^{k+1}\right)$, onde $\tilde{K}^{k+1}$ é uma subvariedade de $S^{m+1}$ de dimensão $k+1$ mergulhada suavemente. Por construção, existe uma fibração $\tilde{\pi}: S^{m+1} \backslash \tilde{K}^{k+1} \rightarrow S^{m-k-1}$ que se restringe a $\pi$ sobre $\left(S^{m} \backslash\left(\operatorname{Int} D \cup K^{k}\right)\right) \times\{t\}$ para cada $t \in S^{1}$. É simples ver que $\left(S^{m+1}, \tilde{K}^{k+1}\right)$ é um NS-par. Ele é chamado o spun do NS-par $\left(S^{m}, K^{k}\right)$. Note que a fibra $\tilde{F}^{k+2}$ da fibração $S^{m+1} \backslash \operatorname{Int} N\left(\tilde{K}^{k+1}\right) \rightarrow S^{m-k-1}$ é difeomorfa à variedade $(k+2)$-dimensional obtida de $F^{k+1} \times S^{1}$ identificando, para cada $x \in \Delta^{k}$, os pontos da forma $(x, t)$ com um ponto para todo $t$, onde $\Delta^{k}$ é um $k$-disco mergulhado em $\partial F^{k+1}$ (perto de $q$ ). Note que os grupos fundamentais de $S^{m} \backslash K^{k}$ e $S^{m+1} \backslash \tilde{K}^{k+1}$ são isomorfos, e que $F^{k+1}$ e $\tilde{F}^{k+2}$ também têm grupos fundamentais isomorfos.

\subsection{Buquê de esferas - caso complexo}

Uma vez que estaremos utilizando ferramentas da Topologia Algébrica, faz-se necessário relembrarmos alguns resultados.

Primeiro, notemos que a esfera $S^{n-1}$ é orientada como o bordo de $D^{n}$, isto é, a classe fundamental $\left[S^{n-1}\right] \in H_{n-1}\left(S^{n-1}\right)$ é definida por $\left[S^{n-1}\right]=\delta\left(\left[D^{n}, S^{n-1}\right]\right)$ onde $\delta: H_{n}\left(D^{n}, S^{n-1}\right) \stackrel{\cong}{\rightarrow} H_{n-1}\left(S^{n-1}\right)$ é o homomorfismo conectante na sequência exata longa para o par $\left(D^{n}, S^{n-1}\right)$.

A aplicação de Hurewicz $\rho: \pi_{n}(X) \rightarrow H_{n}(X)$ é definida por $\rho([\phi])=\phi_{*}\left(\left[S^{n}\right]\right)$, onde $\phi: S^{n} \rightarrow X$ representa um elemento de $\pi_{n}(X),\left[S^{n}\right] \in H_{n}\left(S^{n}\right) \cong \mathbb{Z}$ é o gerador (dado pela orientação natural de $\left.S^{n}\right)$ e $\phi_{*}: H_{n}\left(S^{n}\right) \rightarrow H_{n}(X)$ a aplicação induzida.

Um outro fato importante é que as inclusões $X_{\alpha} \hookrightarrow \bigvee^{\alpha} X_{\alpha}$ induzem o isomorfismo

$$
\bigoplus_{\alpha} \tilde{H}_{q}\left(X_{\alpha}\right) \stackrel{\cong}{\longrightarrow} \tilde{H}_{q}\left(\bigvee^{\alpha} X_{\alpha}\right) \text {. }
$$

Já vimos na Seção 2.2 que a fibra $F_{\theta}$ da fibração de Milnor associada a um germe de função holomorfa $\psi:\left(\mathbb{C}^{n+1}, 0\right) \rightarrow(\mathbb{C}, 0)$ com uma singularidade isolada na origem é equivalente homotópica a um buquê de esferas $n$-dimensionais. 
A seguir, apresentaremos o enunciado e a prova deste resultado conforme [39, pp.57$58]$.

Teorema 5.2.1. A fibra $F_{\theta}$ tem o tipo de homotopia de um buquê de esferas da forma $S^{n} \vee \cdots \vee S^{n}$.

Demonstração. Inicialmente, note que o grupo de homologia $H_{n}\left(F_{\theta}\right)$ é abeliano livre, pois $F_{\theta}$ tem o tipo de homotopia de um CW-complexo de dimensão $n$ (para maiores detalhes, ver [24]). Para $n \geqslant 2$, temos, pelo Lema 2.2.2 e pelo Teorema 1.1.7, que $\pi_{n}\left(F_{\theta}\right) \cong H_{n}\left(F_{\theta}\right)$. Logo, $\pi_{n}\left(F_{\theta}\right)$ é abeliano livre. Assim, é possível escolher um número finito de aplicações $\left(S^{n}, x_{0}\right) \rightarrow\left(F_{\theta}, y_{0}\right)$ para formar uma base, onde $x_{0}$ e $y_{0}$ são pontos base. A wedge das aplicações escolhidas é a aplicação $S^{n} \vee \cdots \vee S^{n} \rightarrow F_{\theta}$, a qual induz um isomorfismo de grupos de homologia (pois a induzida em homologia aplica gerador em gerador conforme discussão feita no início desta seção) e portanto, pelo Teorema de Whitehead, é uma equivalência de homotopia.

Para o caso $n=1$, lembre que uma variedade com bordo tem o mesmo tipo de homotopia do seu interior. Desta forma, podemos considerar $\bar{F}_{\theta}$, a qual sendo uma superfície compacta, orientável e com bordo não-vazio, tem o tipo de homotopia de um buquê de círculos (ver detalhes na Subseção 5.3.1).

A figura a seguir representa um buquê de esferas de mesma dimensão.

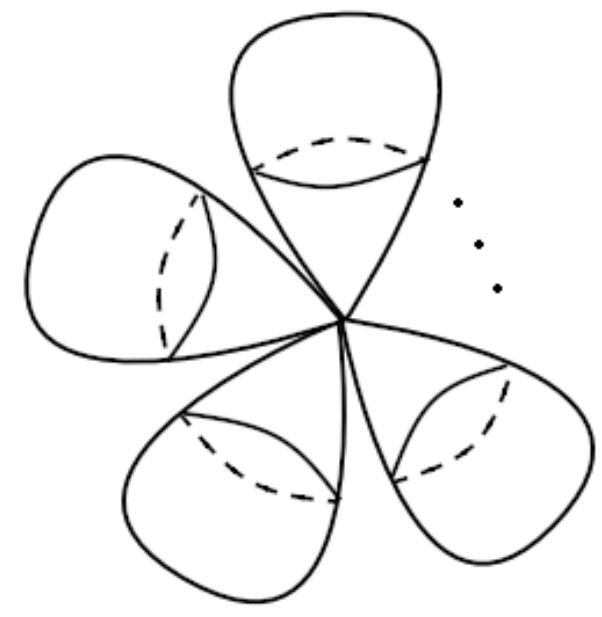

\subsection{Teorema do Buquê - caso real}

Ao longo desta seção consideramos $f:\left(\mathbb{R}^{n}, 0\right) \rightarrow\left(\mathbb{R}^{p}, 0\right), n>p \geqslant 2$, um germe de aplicação polinomial com uma singularidade isolada na origem e a fibração de Milnor (o 
"tubo de Milnor"),

$$
f: f^{-1}\left(S_{\delta}^{p-1}\right) \cap B_{\varepsilon}^{n} \rightarrow S_{\delta}^{p-1}
$$

onde $0<\delta \ll \varepsilon \ll 1$. Denotamos por $F_{f}$ sua fibra e por $\beta_{j}=\operatorname{rank} H_{j}\left(F_{f}\right)$ seu $j$-ésimo número de Betti.

Nas seguintes subseções, consideramos os pares de dimensões $(2 n, n)$ e $(2 n+1, n)$, e estudamos condições para uma fibra de Milnor ter o tipo de homotopia de um buquê de esferas. Também estudamos os pares de dimensões $(2 n, p)$ e $(2 n+1, p)$ com $2 \leqslant p \leqslant n$ usando a composição com uma projeção.

\subsubsection{O caso $(2 n, n)$}

Considere $f:\left(\mathbb{R}^{2 n}, 0\right) \rightarrow\left(\mathbb{R}^{n}, 0\right), n \geqslant 2, f(x)=\left(f_{1}(x), f_{2}(x), \ldots, f_{n}(x)\right)$, um germe de aplicação polinomial com uma singularidade isolada na origem. Note que $S^{2 n-1}$ não fibra suavemente sobre $S^{n-1}$. Portanto, neste caso, $F_{f}$ é uma variedade $n$-dimensional orientável, compacta, com bordo não-vazio e $\pi_{i}\left(F_{f}\right)=0$ para $i=0,1, \ldots, n-2$. Visto que $\partial F_{f} \neq \emptyset$, temos $H_{n}\left(F_{f}\right)=0$. Além disso, como $F_{f}$ é orientável, a homologia $H_{n-1}\left(F_{f}\right)$ é livre de torção. Então, pela Proposição 1.3.3, item (i), temos $\beta_{n-1}=(-1)^{n} \operatorname{deg}_{0}\left(\nabla f_{1}\right)$.

No caso especial $n=2$, as fibras são superfícies orientáveis, conexas, compactas, com bordo não-vazio. Mas, qualquer superfície nessas condições tem o tipo de homotopia de um wedge de círculos (buquê de 1-esferas). De fato, qualquer superfície orientável, compacta com bordo é homeomorfa a um disco com faixas grudadas. Mais precisamente, as extremidades das faixas são grudadas ao disco ao longo do bordo (sem que haja sobreposição). Como o disco pode ser contraído, o resultado segue. Maiores detalhes podem ser encontrados em [36, Seção 12, p.43] e parte da figura a seguir foi retirada de [36, p.44].
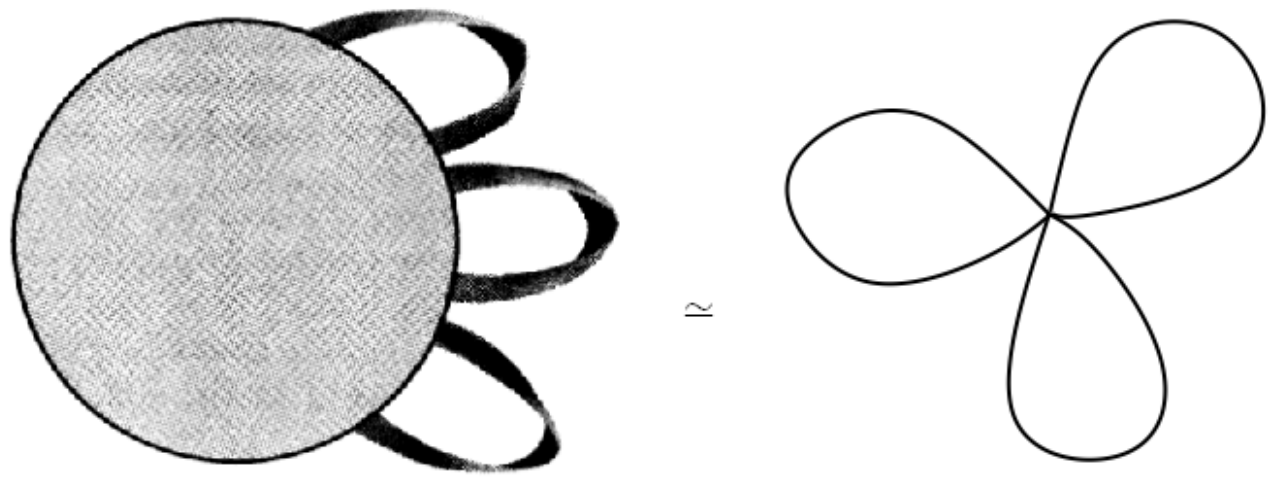

Method of pasting strips to a disc. 
Além disso, para $n=3$, vimos na Seção 4.1 que as fibras são difeomorfas a $S_{(k+1)}^{3}$ para algum inteiro não negativo $k$, e assim, elas são equivalentes homotópicas a um buquê de 2 -esferas. Portanto, podemos assumir que $n \geqslant 4$. Note ainda que se $\operatorname{deg}_{0}\left(\nabla f_{1}\right)=0$, então a fibra de Milnor é contrátil.

Segue do teorema de Hurewicz que o homomorfismo de Hurewicz

$$
\rho_{n-1}: \pi_{n-1}\left(F_{f}\right) \rightarrow H_{n-1}\left(F_{f}\right) \cong \mathbb{Z}^{\beta_{n-1}}
$$

é um isomorfismo. Então, para cada gerador $\gamma_{i} \in H_{n-1}\left(F_{f}\right) \cong \mathbb{Z}^{\beta_{n-1}}$ existe uma aplicação contínua $\varphi_{i}: S^{n-1} \rightarrow F_{f}, i=1,2, \ldots, \beta_{n-1}$, tal que $\gamma_{i}=\rho_{n-1}\left(\left[\varphi_{i}\right]\right)=\left(\varphi_{i}\right)_{*}\left(\left[S^{n-1}\right]\right)$, onde $\left[S^{n-1}\right] \in H_{n-1}\left(S^{n-1}\right) \cong \mathbb{Z}$ é a classe fundamental (dada pela orientação natural de $S^{n-1}$ ). Portanto, temos a aplicação contínua

$$
\varphi: \bigvee^{\beta_{n-1}} S^{n-1} \rightarrow F_{f}
$$

obtida pelo wedge das aplicações $\varphi_{i}: S^{n-1} \rightarrow F_{f}$, para $i=1,2, \ldots, \beta_{n-1}$, a qual é uma equivalência de homotopia pelo teorema de Whitehead. Desta forma, acabamos de provar o seguinte.

Proposição 5.3.1. Seja $f:\left(\mathbb{R}^{2 n}, 0\right) \rightarrow\left(\mathbb{R}^{n}, 0\right)$ um germe de aplicação polinomial com uma singularidade isolada na origem, $n \geqslant 2$. Dado $f(x)=\left(f_{1}(x), f_{2}(x), \ldots, f_{n}(x)\right)$, um representante do germe $f$, temos o seguinte.

(i) $\beta_{n-1}=(-1)^{n} \operatorname{deg}_{0}\left(\nabla f_{1}\right)$.

(ii) A fibra de Milnor $F_{f}$ tem o tipo de homotopia de um buquê de esferas $(n-1)$ dimensionais

$$
\bigvee_{i=1}^{\beta_{n-1}} S_{i}^{n-1},
$$

o qual representa um ponto quando $\beta_{n-1}=0$.

Para $n \geqslant 4$, segue do Teorema 2.3.4, item (b), que em todos os pares de dimensões $(2 n, n)$ existem exemplos não-triviais. No entanto, esses exemplos não-triviais devidos a Church-Lamotke [12] têm fibras de Milnor contráteis (com links não simplesmente conexos). Por outro lado, conforme nossa construção na Seção 4.3 (mais precisamente, Corolário 4.3.2) e o Teorema 2.3.2, obtemos o seguinte.

Corolário 5.3.2. Para cada par de dimensões $(2 n, p), 2 \leqslant p \leqslant n$, existe um germe de aplicação polinomial real com singularidade isolada $\left(\mathbb{R}^{2 n}, 0\right) \rightarrow\left(\mathbb{R}^{p}, 0\right)$ tal que a fibra de Milnor é, a menos de homotopia, um buquê de esferas $(n-1)$-dimensionais com o número de esferas igual a $\left|\operatorname{deg}_{0}\left(\nabla f_{1}\right)\right|>0$, onde $f(x)=\left(f_{1}(x), f_{2}(x), \ldots, f_{p}(x)\right)$. 
Demonstração. O Corolário 4.3.2 garante a existência de germes de aplicações polinomiais reais com singularidade isolada $\left(\mathbb{R}^{2 n}, 0\right) \rightarrow\left(\mathbb{R}^{n}, 0\right)$ que são não-triviais. Pela Proposição 5.3.1, a fibra de Milnor desses exemplos tem o tipo de homotopia de um buquê de um número positivo de esferas $(n-1)$-dimensionais. Agora, aplicando o Teorema 2.3.2, vemos que o tipo de homotopia da fibra é preservado após composição da aplicação com a projeção.

Observação 5.3.3. Note que no caso de germes de aplicações polinomiais reais com singularidade isolada, $\left(\mathbb{R}^{2 n}, 0\right) \rightarrow\left(\mathbb{R}^{2}, 0\right)$, provenientes de germes de funções holomorfas, a fibra de Milnor associada tem o tipo de homotopia de um buquê de esferas $(n-1)$-dimensionais. No entanto, os exemplos referidos na Proposição 5.3.1, $n \geqslant 3$, não vêm, necessariamente, de ambiente complexo.

\subsubsection{O caso $(2 n+1, n)$}

Observe que pela Proposição 1.3.3, item (ii), nesses pares de dimensões, a fibra não pode ter o tipo de homotopia de um buquê de esferas de mesmas dimensões. Então, surge a pergunta natural:

Sob quais condições podemos garantir que a fibra tem o tipo de homotopia de um buquê de esferas de diferentes dimensões?

Para responder esta pergunta, considere agora $f:\left(\mathbb{R}^{2 n+1}, 0\right) \rightarrow\left(\mathbb{R}^{n}, 0\right), n \geqslant 3$, um germe de aplicação polinomial com uma singularidade isolada na origem. Neste caso, a fibra de Milnor $F_{f}$ é uma variedade $(n+1)$-dimensional, compacta, orientável, com bordo não-vazio e é $(n-2)$-conexa. Então, $H_{n+1}\left(F_{f}\right)=0, H_{n}\left(F_{f}\right)$ é livre de torção e, pela Proposição 1.3.3, $\beta_{n}=\beta_{n-1}$. Suponha que $H_{n-1}\left(F_{f}\right)$ é livre de torção. Então, temos $H_{n-1}\left(F_{f}\right) \cong \mathbb{Z}^{\beta_{n-1}} \cong H_{n}\left(F_{f}\right)$. Pelo teorema de Hurewicz (Teorema 1.1.7), os homomorfismos de Hurewicz

$$
\rho_{n-1}: \pi_{n-1}\left(F_{f}\right) \rightarrow H_{n-1}\left(F_{f}\right) \cong \mathbb{Z}^{\beta_{n-1}}
$$

e

$$
\rho_{n}: \pi_{n}\left(F_{f}\right) \rightarrow H_{n}\left(F_{f}\right) \cong \mathbb{Z}^{\beta_{n-1}}
$$

são sobrejetores. Então, por um argumento similar àquele usado no caso $(2 n, n)$, podemos construir uma equivalência de homotopia

$$
\varphi:\left(\bigvee^{\beta_{n-1}} S^{n}\right) \vee\left(\bigvee^{\beta_{n-1}} S^{n-1}\right) \rightarrow F_{f}
$$

Assim, acabamos de provar o seguinte resultado. 
Proposição 5.3.4. Seja $f:\left(\mathbb{R}^{2 n+1}, 0\right) \rightarrow\left(\mathbb{R}^{n}, 0\right), n \geqslant 3$, um germe de aplicação polinomial real com singularidade isolada. Então, o $(n-1)$-ésimo grupo de homologia $H_{n-1}\left(F_{f}\right)$ da fibra de Milnor é livre de torção se, e somente se, $F_{f}$ tem o tipo de homotopia de um buquê de esferas da forma

$$
\left(\bigvee^{\beta_{n-1}} S^{n-1}\right) \vee\left(\bigvee^{\beta_{n-1}} S^{n}\right)=\bigvee^{\beta_{n-1}}\left(S^{n-1} \vee S^{n}\right)
$$

o qual representa um ponto quando $\beta_{n-1}=0$.

A figura a seguir representa um buquê de esferas de diferentes dimensões.

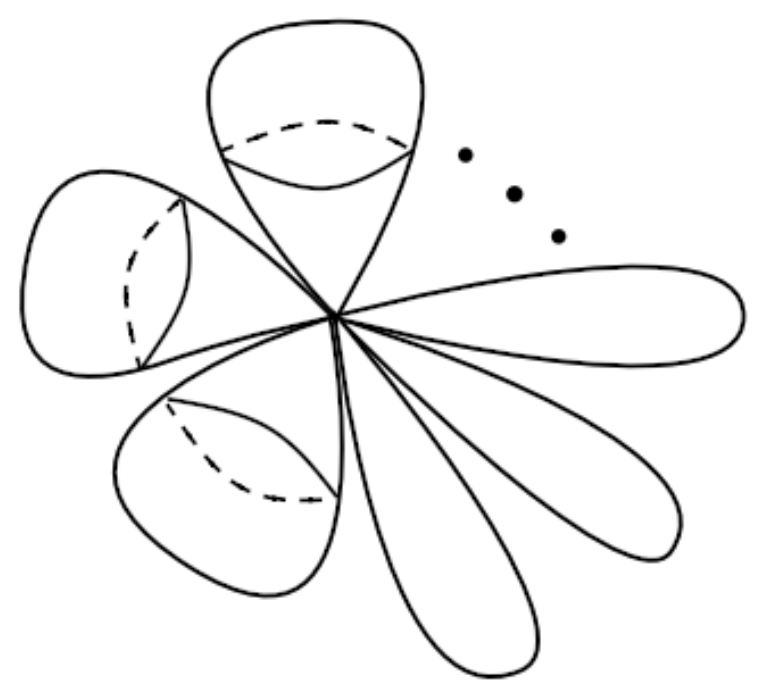

Mais uma vez considerando a nossa construção na Seção 4.3 e o Teorema 2.3.2, obtemos o seguinte.

Corolário 5.3.5. Para cada par de dimensões $(2 n+1, p), 2 \leqslant p \leqslant n$, existe um germe de aplicação polinomial real com singularidade isolada $\left(\mathbb{R}^{2 n+1}, 0\right) \rightarrow\left(\mathbb{R}^{p}, 0\right)$ tal que a fibra de Milnor é, a menos de homotopia, um buquê de $\ell$ cópias da esfera $n$-dimensional e $\ell$ cópias da esfera $(n-1)$-dimensional com $\ell>0$.

Demonstração. Para $n \geqslant 3$, isto é uma consequência da Proposição 5.3.4. Para $n=2$, começamos com um nó fibrado não-trivial $\left(S^{3}, K\right)$. Então, seu spun $\left(S^{4}, \tilde{K}\right)$ é um 2-nó fibrado não-trivial, e sua fibra é obtida do spinning de uma superfície com bordo e de gênero positivo. Portanto, a fibra de $\left(S^{4}, \tilde{K}\right)$ tem o tipo de homotopia de um buquê de um número positivo de círculo e 2-esferas. Isso completa a prova. 


\subsection{Aplicação $k$-stairs}

Dado um germe de aplicação polinomial $f:\left(\mathbb{R}^{n}, 0\right) \rightarrow\left(\mathbb{R}^{q}, 0\right), n \geqslant q \geqslant 1$, com uma singularidade isolada na origem, dizemos que um germe de aplicação $F:\left(\mathbb{R}^{n}, 0\right) \rightarrow\left(\mathbb{R}^{p}, 0\right), 1 \leqslant q \leqslant p$, é uma aplicação $(p-q)$-stairs para $f$ se existem germes de funções polinomiais $g_{j}:\left(\mathbb{R}^{n}, 0\right) \rightarrow(\mathbb{R}, 0), q+1 \leqslant j \leqslant p$, tais que $F(x)=\left(f(x), g_{q+1}(x), g_{q+2}(x), \ldots, g_{p}(x)\right)$ tem uma singularidade isolada na origem. Se $p=q$, então por definição, temos que $F(x)=f(x)$ e $f$ é sua própria aplicação 0-stairs.

Corolário 5.4.1. Seja $f:\left(\mathbb{R}^{n}, 0\right) \rightarrow\left(\mathbb{R}^{p}, 0\right), n / 2 \geqslant p \geqslant 2$, um germe de aplicação polinomial com uma singularidade isolada na origem. Então temos o seguinte.

(i) Se $n$ é par e $f$ admite um germe de aplicação $(n / 2-p)$-stairs, então a fibra de Milnor é equivalente homotópica a um buquê de esferas $(n / 2-1)$-dimensionais.

(ii) Suponha $n$ impar e que $H_{k}\left(F_{f}\right)$ é livre de torção para $k=(n-1) / 2-1$, onde $F_{f}$ denota a fibra de Milnor. Se $f$ admite um germe de aplicação $((n-1) / 2-p)$ stairs, então a fibra de Milnor é equivalente homotópica a um buquê de esferas $k$ - $e$ $(k+1)$-dimensionais, onde os números de esferas são iguais.

Demonstração. Basta aplicar as Proposições 5.3.1, 5.3.4, e o Teorema 2.3.2. 


\section{Referências Bibliográficas}

[1] Araújo dos Santos, R. Equivalence of real Milnor fibrations for quasihomogeneous singularities. Rocky Mountain Journal of Mathematics 42, 2 (2012), 439-449.

[2] Araújo dos Santos, R., Chen, Y., And Tibăr, M. Singular open book structures from real mappings. Cent. Eur. J. Math. 11, 5 (2013), 817-828.

[3] Araújo dos Santos, R., Chen, Y., And Tibăr, M. Real polynomial maps and singular open books at infinity. arXiv preprint arXiv:1401.8286 (2014).

[4] Araújo dos Santos, R., Dreibelbis, D., And Dutertre, N. Topology of the real Milnor fiber for isolated singularities. In Real and complex singularities, vol. 569 of Contemp. Mathematics. Amer. Math. Soc., Providence, RI, 2012, pp. 67-75.

[5] Araújo dos Santos, R., Hohlenwerger, M. A. B., Saeki, O., and Souza, T. O. New examples of Neuwirth-Stallings pairs and non-trivial real Milnor fibrations. arXiv preprint arXiv:1406.2030 (2014).

[6] Araújo dos Santos, R., And TibăR, M. Real map germs and higher open book structures. Geom. Dedicata 147 (2010), 177-185.

[7] Artin, E. Zur isotopie zweidimensionaler flächen im $\mathbb{R}^{4}$. In Abhandlungen aus dem Mathematischen Seminar der Universität Hamburg (1925), vol. 4, Springer, pp. 174177.

[8] Bredon, G. E. Topology and geometry, vol. 139 of Graduate Texts in Mathematics. Springer-Verlag, New York, 1993.

[9] Bröcker, T., And tom Dieck, T. Representations of compact Lie groups, vol. 98. Springer, 1985.

[10] Browder, W. Higher torsion in h-spaces. Transactions of the American Mathematical Society 108 (1963), 353-375. 
[11] CERF, J. Sur les difféomorphismes de la sphère de dimension trois $\left(\Gamma_{4}=0\right)$. No. 53 in Lectures Notes in Mathematics. Springer, 1968.

[12] Church, P. T., And Lamotke, K. Non-trivial polynomial isolated singularities. Nederl. Akad. Wetensch. Proc. Ser. A 78=Indag. Math. 37 (1975), 149-154.

[13] DimcA, A. Singularities and topology of hypersurfaces. Springer, 1992.

[14] Dutertre, N. Degree formulas for a topological invariant of bifurcations of functiongerms. Kodai Mathematical Journal 23, 3 (2000), 442-461.

[15] Dutertre, N. On the Euler characteristics of real Milnor fibres of partially parallelizable maps of $\left(\mathbb{R}^{n}, 0\right)$ to $\left(\mathbb{R}^{2}, 0\right)$. Kodai Mathematical Journal 32, 2 (2009), $324-351$.

[16] Dutertre, N., and Araújo dos Santos, R. Topology of real Milnor fibration for non-isolated singularities. arXiv preprint arXiv:1211.6233 (2012).

[17] Dutertre, N., Araújo dos Santos, R., Chen, Y., And Andrade, A. Fibrations structure and degree formulae for Milnor fibers. arXiv preprint arXiv:1409.5053 (2014).

[18] Dutertre, N., Araújo dos Santos, R., Chen, Y., And Andrade, A. Open book structures on semi-algebraic manifolds. arXiv preprint arXiv:1409.4316 (2014).

[19] Fadell, E. R., And Husseini, S. Y. Geometry and topology of configuration spaces. Springer, 2001.

[20] FunAR, L. Global classification of isolated singularities in dimensions $(4,3)$ and (8,5). Ann. Scuola Norm. Sup. Pisa Cl. Sci. 10 (2011), 819-861.

[21] Haefliger, A. Differentiable links. Topology 1, 3 (1962), 241-244.

[22] Hamm, H. A., And Tráng, L. Un théorème de Zariski du type de Lefschetz. In Annales Scientifiques de l'École Normale Supérieure (1973), vol. 6, Société Mathématique de France, pp. 317-355.

[23] Hatcher, A. A proof of the Smale conjecture, $\operatorname{Diff}\left(S^{3}\right) \simeq O(4)$. Annals of Mathematics 117 (1983), 553-607.

[24] Hatcher, A. Algebraic topology. Cambridge University Press, Cambridge, 2002.

[25] HatcheR, A. Vector bundles and K-theory. http://www.math.cornell.edu/ hatcher/ (2003). 
[26] JaCquemard, A. Sur les singularités des applications polynomiales réelles. Thèse 3ème cycle Université de Dijon (1982).

[27] Jacquemard, A. Fibrations de Milnor pour des applications réelles. Boll. Un. Mat. Ital 37, 1 (1989), 45-62.

[28] Kato, M., and Matsumoto, Y. On the connectivity of the Milnor fiber of a holomorphic function at a critical point. Manifolds-Tokyo (Proc. Internat. Conf., Tokyo, 1973) (1973), 131-136.

[29] Kervaire, M. A., And Milnor, J. W. Groups of Homotopy Spheres: I. Annals of Mathematics r7, 3 (1963), 504-537.

[30] Khimshiashvili, G. N. The local degree of a smooth mapping. Sakharth. SSR Mecn. Akad. Moambe 85, 2 (1977), 309-312.

[31] KIM, S. Local triviality of completely regular mappings. Duke Mathematical Journal 38,3 (1971), 467-471.

[32] LEE, J. Introduction to smooth manifolds, vol. 218. Springer, 2012.

[33] Eojasiewicz, S., And Zurro, M.-A. On the gradient inequality. Bulletin of the Polish Academy of Sciences-Mathematics 47, 2 (1999), 143-146.

[34] Looijenga, E. A note on polynomial isolated singularities. Nederl. Akad. Wetensch. Proc. Ser. A 74=Indag. Math. 33 (1971), 418-421.

[35] Massey, D. B. Real analytic Milnor fibrations and a strong Łojasiewicz inequality. In Real and complex singularities, vol. 380 of London Math. Soc. Lecture Note Series. Cambridge Univ. Press, Cambridge, 2010, pp. 268-292.

[36] Massey, W. S. Algebraic topology, an introduction. Springer-Verlag (New York), 1977.

[37] Milnor, J. Lectures on the h-cobordism theorem, vol. 390. Princeton University Press, Princeton, NJ, 1965.

[38] Milnor, J. Topology from the differentiable viewpoint. Univ. Press Virginia, 1965.

[39] Milnor, J. Singular points of complex hypersurfaces. Annals of Mathematics Studies, No. 61. Princeton University Press, Princeton, N.J., 1968.

[40] Outerelo, E., And Ruiz, J. M. Mapping degree theory, vol. 108. American Mathematical Soc., 2009. 
[41] Palais, R. S. Local triviality of the restriction map for embeddings. Commentarii Mathematici Helvetici 34, 1 (1960), 305-312.

[42] Rolfsen, D. Knots and links, vol. 7. Publish or perish Berkeley, 1976.

[43] Ruas, M. A. S., Seade, J., And Verjovsky, A. On real singularities with a Milnor fibration. Birkhäuser Basel, 2002, pp. 191-213.

[44] SeAde, J. On the topology of isolated singularities in analytic spaces, vol. 241 of Progress in Mathematics. Birkhäuser Verlag, Basel, 2006.

[45] Smale, S. Generalized Poincaré's conjecture in dimensions greater than four. Ann. of Math 74, 2 (1961), 391-406.

[46] SouzA, T. O. Teoremas de (H, G)-coincidências para variedades e classificação global de singularidades isoladas em dimensões $(6,3)$. $\mathrm{PhD}$ thesis, Universidade de São Paulo, 2013.

[47] Souza, T. O., Hohlenwerger, M. A. B., De Mattos, D., and Araújo Dos SAntos, R. New characterization of trivial maps in 3-dimensional real Milnor fibers. JP J. Geom. Topol. 12, 2 (2012), 207-217.

[48] Spanier, E. H. Algebraic Topology. McGraw-Hill (New York), 1966.

[49] Switzer, R. M. Algebraic topology-homotopy and homology. Springer Berlin, 1975.

[50] VICK, J. W. Homology theory: an introduction to algebraic topology, vol. 145 of Graduate Texts in Mathematics. Springer-Verlag, New York, 1994.

[51] Zeeman, E. C. Twisting spun knots. Trans. Amer. Math. Soc 115 (1965), 471-495. 